Literature Reviews

\title{
State of the Art on Video Streaming and Applications
}

\author{
${ }^{1}$ Yahia Hasan and ${ }^{2}$ Ali Zanbouri \\ ${ }^{1}$ Department of ITC, Arab Open University, Kuwait \\ ${ }^{2}$ Department of Computer Science, Algonquin College, Kuwait
}

\author{
Article history \\ Received: 31-01-2018 \\ Revised: $13-02-2018$ \\ Accepted: 20-04-2018 \\ Corresponding Author: \\ Yahia Hasan \\ Department of ITC, Arab Open \\ University, Kuwait \\ Email: yahia@aou.edu.kw
}

\section{Introduction}

As a matter of fact that we are living in the century of accelerated and continuous events and news; therefore enhancing the quality of real-time and live video streaming (Ghareeb, 2008) over the packet erasure (or best efforts) wired and wireless networks become shot topic nowadays.

Video and video streaming ideas take us back to the year 1951 when Charles Ginsburg developed the first practical videotape recorder (VTR) (The History of Video and Related Innovations). After that the Video

\begin{abstract}
The real-time and live video streaming are playing an important role in our daily lives; therefore enhancing the quality of realtime and live video streaming over wired and wireless packet erasure networks which becomes more and more important topic nowadays. The main objective of this tutorial paper is to highlight state of the art on video streaming and the most important methods for enhancing video streaming over wired and wireless packet erasure networks to the computer/communication engineering students and new researchers. Firstly, it presents an overview of layered coding compression techniques (like: H.264/SVC) which lead to better real-time video streaming quality than single layer coding techniques (like: H.264/AVC). Secondly, it highlights how to solve the channel errors and lost packets problems in video streaming via implementing some of Forward Error Correction (FEC) techniques. Furthermore, it presents Backward Error Correction (BEC) techniques for enhancing the video streaming. After that it talks about the implementation of rateless FEC techniques with feedback for solving the real-time and live video streaming issues. Then it presents some recent researches on the Unequal Error (or Loss) Protection (UEP) techniques which are more powerful and achieve better Peak Signal to Noise Rate (PSNR) than the previous Equal Error Protection (EEP) techniques. Also it highlights the adaptive UEP scheme which brings significant improvements in terms of average PSNR over static UEP scheme for same used bandwidth. Furthermore, it talks about the multipath video streaming which leads to better loss characteristics and better load distribution on wireless networks than the single path video streaming. Finally, it presents some recent researches that based on hybrid solution of rateless FEC and advanced BEC techniques for enhancing the real-time and live video streaming quality over $3 \mathrm{G}, \mathrm{Wi}-\mathrm{Fi}$ and WiMAX wireless networks.
\end{abstract}

Keywords: Video Streaming, Compression Techniques
Home System (VHS) or Video tape in a large cassette format was developed by both JVC and Panasonic companies in 1976 (The History of Video and Related Innovations). Then in 1991, the video signals started to be processed by high-speed Digital Signal Processors (DSPs) and Single Instruction stream Multiple Data stream (SIMD) technologies (Kuroda and Nishitani, 1998). In 1994, a Video On Demand (VOD) service considered as a crucial application on the Cambridge Digital Interactive Television Trial-network in UK (Greaves and Taunton, 1996), notice that VOD services (like watching a stored video movie) can tolerate some 
delay up to tens of seconds between requesting and playing out the stored video streaming, therefore the video contents can be encoded offline (Ghareeb, 2008).

Then some bandwidth control mechanisms were proposed to solve the congestion and network overloading problems in Replicated-Stream Multicast Video Distribution (Li and Ammar, 1996). After that in early 1997, the first Practical Real Video streaming was introduced by Robert Glaser (FU, 2011).

However the most important ideas on enhancing the quality of video streaming over wired and wireless packet erasure networks were proposed after the year 1997. First researches concentrated on how to solve the congestion and lost packets problems in video streaming by implementing Backward Error Correction (BEC) techniques like TCP (Elaywe and Kamal, 2002), in BEC the receiver can discover the errors then it sends a request (or feedback) for the sender to retransmit the corrupted or lost data, But on the other hand they increased the delay on receiving the video streaming. With comparison to the non-streaming media applications (like: Text, images and graphics), the realtime video-streaming applications are very sensitive to delay and can tolerate some packets losses (Ghareeb, 2008). Using Forward Error Correction (FEC) techniques is one possible solution to the delay and packets losses problems in real-time video streaming over packet erasure channels (Wang et al., 2002), in FEC the receiver can detect and fix the errors directly without retransmission the corrupted data. However, the FEC techniques lead to bandwidth expansion (or increasing bandwidth overhead) and hence increase the lost packets rates, therefore these factors lead to reduce the Peak Signal to Noise Ratio (PSNR). Then they used rateless FEC codes with feedbacks based on packet loss rate histogram (Ahmad et al., 2007) in order to reduce FEC bandwidth overhead while keeping successful reconstruction of the video streaming and hence results in enhancing the PSNR and decreasing the congestion level.

In (Mohr et al., 2000), the Unequal Error Protection (UEP) techniques were introduced as effective solutions to achieve better PSNR than the Equal Error Protection (EEP) techniques over wireless packet erasure networks. In UEP the transmitted information are varied in their importance (most important and least important information), whereas in EEP all the information have the same priority level. Furthermore, it has been proved that UEP techniques are more suitable for the applications where high video quality is required.

However, the recent researches focus on hybrid solutions between advanced BEC and rateless FEC techniques to solve the real-time and live video streaming issues over $3 \mathrm{G}, \mathrm{Wi}-\mathrm{Fi}$ and WiMAX wireless networks. While the live video services (like watching live football match) can tolerate some delay up to ten seconds, the real-time video services (like video conferencing) cannot accept delay more than 400 milliseconds between requesting and playing out the video streaming. However in both cases, the video contents should be encoded in real-time.

This tutorial paper highlights clear and sufficient ideas about the video streaming and the most important methods for enhancing it over wired and wireless lossy packet networks to the computer/communication engineering students and new researchers.

The packet erasure channel is a communication channel model where sequential packets are either received or lost. This channel model is closely related to the binary erasure channel. An erasure code can be used for forward error correction on such a channel.

The communication link in packet erasure network is the erasure channel. When no enough packets can reach the destination, no packet ca recover; an erasure in network coding is more severe than a classical erasure since one erased packet may contaminate other packets.

The rest of this tutorial paper is organized as follows: In section II we briefly go through the history of video and video streaming. Then in Section III, we present an overview of video streaming compression standards. In section IV we present the differences between FEC and BEC techniques. Section V demonstrates FEC codes could be used with feedbacks in order to enhance the video streaming quality. After that section VI presents how to enhance the video streaming quality via implementing the UEP techniques. Then in section VII the Layered Video Streaming is discussed. After that in section VIII we describe the differences between single path and multiple paths video-streaming techniques. Then section IX introduces how to apply the real-time and live video streaming over $3 \mathrm{G}$, Wi-Fi and WiMAX wireless networks. Finally we show the summary and conclusion parts.

\section{History of Video and Video Streaming}

In 1951, Charles Ginsburg developed the first practical Video Tape Recorder (VTR). This device converts the live images which captured by the television cameras into electrical impulses and then it saves this information onto magnetic tape (The History of Video and Related Innovations).

In 1976, the Video Home System (VHS) or Video tape in a large cassette format was developed by both JVC and Panasonic companies. In addition VHS was the most famous format for home use and video store rentals until 1997 then it was replaced by mini DV tapes and DVDs (The History of Video and Related Innovations).

In 1981, IBM introduced the first color display standard for the Personal Computers which was called Color Graphics Adapter (CGA); also they added $16 \mathrm{~KB}$ video RAM to this graphic card (Mueller, 1992). Later in 
1987, IBM developed the display hardware standard with name Video Graphics Array (VGA). First VGA card achieved 256 simultaneous colors at a resolution of $320 \times 200$ and 16 colors at $640 \times 480$ (Polsson, 2010).

In 1991, the video signals began to be processed by fast DSP processors (Kuroda and Nishitani, 1998); In addition, the performance enhancement was achieved from higher clocks as well as SIMD architectures. On the other hand, the quarter National Television Systems Committee (NTSC) video format which was used at that time for video-conferencing applications was called Common Intermediate Format (CIF) (or $352 \times 288$ pixels format) (Kuroda and Nishitani, 1998).

In 1993, there was a big step for Moving Picture Experts Group-2(MPEG-2) applications (Kuroda and Nishitani, 1998; Foley, 1996; Rathnam and Slavenburg, 1996), where a full NTSC resolution format was implemented. In that time, SIMD architectures were developed to handle Very Long Instruction Word (VLIW) controls. MPEG-2 applications contain set-top boxes for digital cable TV and Video On Demand (VOD) (Kuroda and Nishitani, 1998).

In September 1994, VOD service considered as crucial application on the Cambridge Digital Interactive Television Trial network in UK. This network supplied up to 250 homes and a number of schools connected to the Cambridge Cable network with VOD services. Furthermore, this project was based on packet switched Asynchronous Transfer Mode (ATM) technology (Greaves and Taunton, 1996).

Li and Ammar (1996), they proposed some bandwidth control mechanisms to solve the congestion and network overloading problems in Replicated-Stream Multicast Video Distribution. It is a matter of fact that carrying replicated streams on network links leads to congestion problem on these links and hence this leads to reduce the quality of video transmission; therefore they added bandwidth control mechanism in (Li and Ammar, 1996) to solve congestion and overloading problems, furthermore this mechanism relied on the feedbacks from the receivers to meet the current network capabilities. But on the other hand this bandwidth control mechanism caused a large delay in the video streaming delivery process; therefore this mechanism was not suitable for the real-time video streaming.

In early 1997, the first Practical Real Video streaming was introduced by Robert Glaser (from Real Networks, Inc. company); also it allows the user to play videos on the Web. Furthermore, this type of Video streaming was based on the H.263 compression format (or video compression standard designed as a low-bit rates compressed format for video conferencing) (FU, 2011).

However, the most important and mature researches on enhancing the video streaming quality over packet erasure networks (e.g., the Internet) were started after the year 1997, It is a matter of fact that these researches were relied on Backward Error Correction (BEC) and Forward Error Correction (FEC) techniques.

In next section, we present an overview of video streaming compression standards and how these standards could compress the raw video and make it ready for the error correction stage. Then we discuss the most important types of FEC and BEC techniques, Furthermore we show some of their important applications in enhancing the quality of video streaming over packet erasure networks.

\section{An Overview of Video Compression Standards}

Compression is a process of reducing the size of data to a more compact form. Therefore, through the compression process portions of redundant data are detected and removed. Indeed, there are two types of data compression techniques: Lossless compression and lossy compression. In lossless compression the original binary data are compressed in such a way that it can be reconstructed exactly later on without loss, whereas in lossy compression the original binary data are compressed in such a way that it cannot be reconstructed exactly later on. However, the lossy compression usually achieves greater compression of the data than the lossless compression; therefore the lossy compression techniques are more suitable for the video streaming applications. In next sub-sections, we present the most important types of lossy compression standards that used in video applications.

\section{Motion Picture Experts Group - 1 (MPEG-1)}

MPEG-1 is the first public standard for the Motion Picture Experts Group committee and it was approved in November 1991. MPEG-1 frames are encoded in three different methods (ACC, 2008; Abomhara et al., 2010):

- Intra-coded frames (I-frames): Here the MPEG frames are encoded as discrete frames and independent of adjacent frames (or each frame has no dependency outside of that image)

- Predictive-coded frames (P-frames): Here each MPEG frame is encoded by prediction from one previous (or past) I-frame or P-frame, hence this method leads to a better compression ratio (or less number of frames) than the I-frames method

- Bi-directional-predictive-coded frames (B-frames): Here each MPEG frame is encoded by prediction from two frames, one previous frame and one subsequent frame of either I-frames or P-frames (as shown in Fig. 1) and therefore out of order decoding is needed. As a matter of fact, this method leads to a better compression ratio (or less number of frames) than the previous two methods 


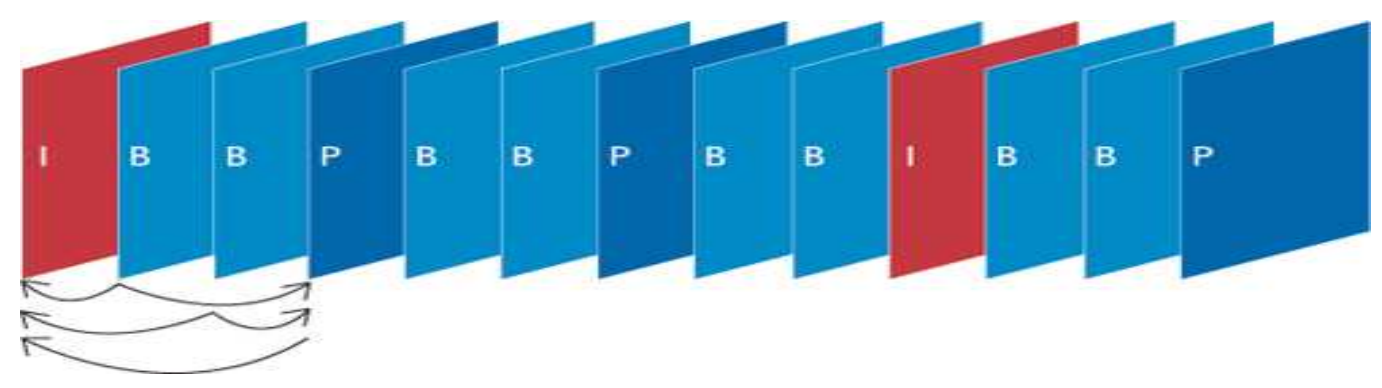

Fig. 1: The diagram above describes how a sequence with I-, P- and B-frames may look like. Note that a P-frame may only reference a previous I- or P-frame, while a B-frame may reference both previous and subsequent I- and P-frames

After that, the video decoder restores the video by decoding the bit stream frame by frame. The decoding process should begin with an I-frame, which can be decoded independently, while P- and B-frames must be decoded together with current reference image(s) (ACC, 2008). By the way, MPEG-1 was suitable for video on digital storage media (i.e., VCDROM) (Abomhara et al., 2010; Jian-Wen et al., 2006).

\section{Motion Picture Experts Group - 2 (MPEG-2)}

MPEG-2project was approved in November 1994. The MPEG-2 project concentrated on enhancing the MPEG-1 compression technique to handle larger pictures and achieve higher quality at the expense of a higher bandwidth usage. MPEG-2 was designed to deal with video applications which require a bit rate of up to 100 Mbps; Digital high-definition TV (HDTV), interactive storage media (ISM) and cable TV (CATV) are examples of these applications. Furthermore, DVD movies compression technique was based on MPEG-2. However, MPEG-2 is not designed for video applications through the Internet as it requires too much bandwidth (ACC, 2008; Abomhara et al., 2010; JianWen et al., 2006; Tosun, 2009; Puri et al., 2004).

\section{Motion Picture Experts Group - 4 (MPEG-4)}

MPEG-4 project was approved in October 1998; MPEG-4 is based on the same technique as MPEG-1 and MPEG-2. Success of digital television, interactive graphics applications and interactive multimedia encouraged MPEG group to design MPEG-4. The most important features of MPEG-4 were (ACC, 2008; Abomhara et al., 2010; Tosun, 2009; Puri et al., 2004):

- It can achieve very good video compression rate for lower bandwidth consuming applications, like mobile phone video applications (Jian-Wen et al., 2006)

- Also it can achieve a good video compression rate for video applications with extremely high quality and almost unlimited bandwidth
From (ACC, 2008; Abomhara et al., 2010; Jian-Wen et al., 2006; Tosun, 2009; Puri et al., 2004) it can be concluded that the MPEG-4 was the first compression technique that developed for the Internet video streaming applications. Furthermore, video conferencing become possible with MPEG-4 standard. But on the other hand, it gives lower video quality than MPEG-1 and MPEG-2, because sending video through the Internet was really a big challenge.

\section{Motion Picture Experts Group - 7 (MPEG-7)}

MPEG-7 was approved in July 2001. In fact this standard does not deal with the actual encoding of moving pictures and audio; However MPEG-7 is a video content description standard (as shown in Fig. 2). Therefore in MPEG-7, the content of the video is described and associated with the content itself;as a result MPEG-7 can achieve fast and efficient searching in the material. Furthermore, MPEG-7 depends on XML to store metadata and it can be attached to a time code in order to select particular events in a video stream (ACC, 2008; Abomhara et al., 2010; Tosun, 2009; Martinez, 2002).

To be more specific, MPEG-7could be used to select the contents and particular events of video streams for smart processing in video management software or video analytics applications. Therefore it becomes suitable for video surveillance applications stream (ACC, 2008; Abomhara et al., 2010; Martinez, 2002).

\section{H.263 Compression Standard}

H.263 was developed by the International Telecommunication Union (ITU) in 1996; H.263 depends on block motion-compensated Discrete Cosine Transform (DCT) structure for encoding. Also it was significantly designed for very low bit rate coding applications (like for video-conferencing over Public Switched Telephone Network (PSTN)). However, H.263 is not suitable for usage in general digital video coding (ACC, 2008; Abomhara et al., 2010; Tosun, 2009). 


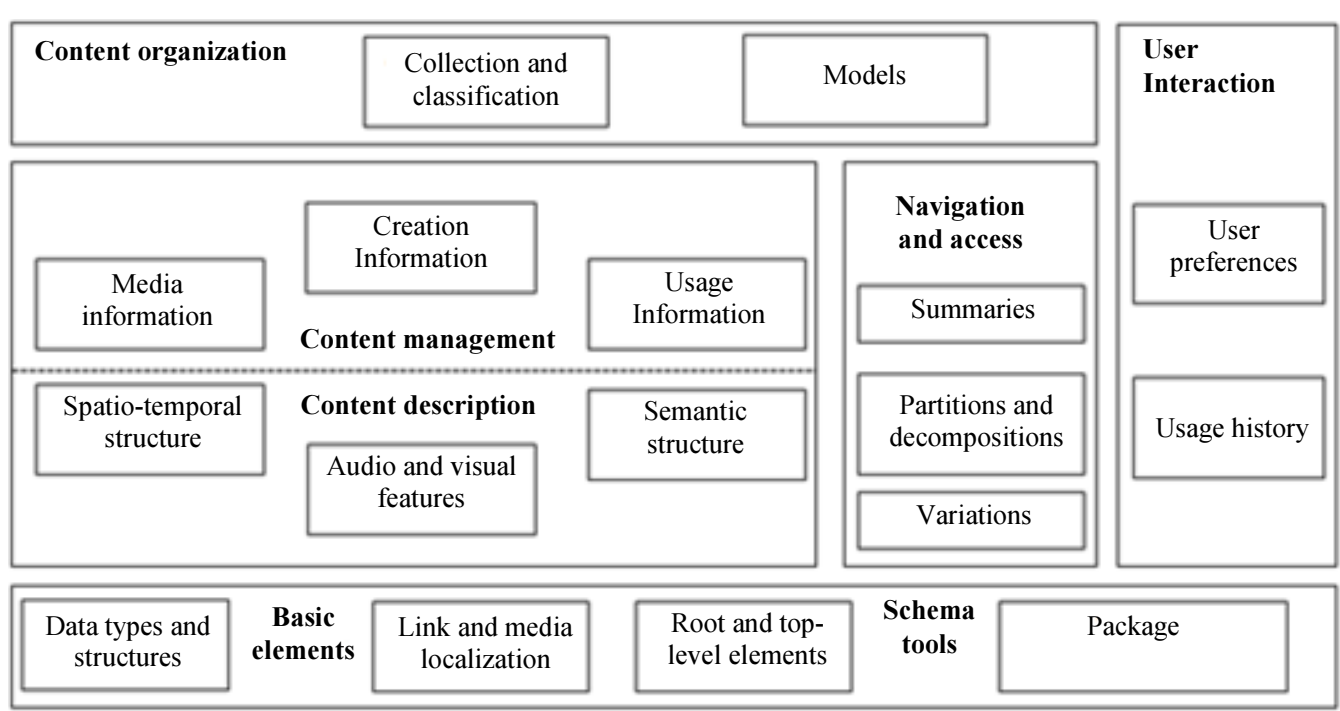

Fig. 2: Summary of MPEG-7 description tools (Martinez, 2002)

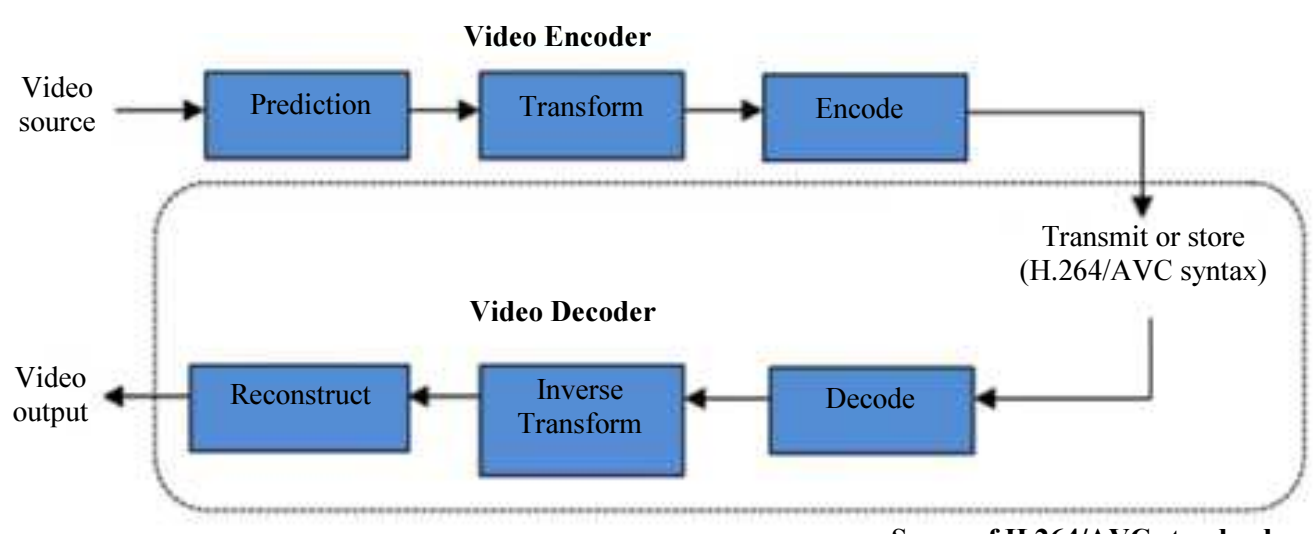

Scope of H.264/AVC standard

Fig. 3: The encoding and decoding processes in H.264/AVC video compression standard (Richardson, 2007-2011)

\section{H.264 Advanced Video Coding (H.264/AVC) Compression Standard}

H.264/AVC (as shown in Fig. 3) was approved by ITU-T in March 2003 (also called MPEG-4 part 10). This standard was designed to provide lower latency, higher quality video streaming (even at low bit rates for storage or transmission), better error robustness and less complexity than the previous compression standards. Also, this standard has enough flexibility to deal wide range of video applications: For both low (such as mobile video conferencing) and high (such as Ultra HDTV) bit rates, for low and high resolution video and with low and high demands of latency (ACC, 2008; Abomhara et al., 2010; Jian-Wen et al., 2006; Puri et al., 2004; Richardson, 2007-2011).

Furthermore, it can be concluded that H.264/AVC video compression standardize an essential technology for many consumer electronics applications, including (ACC, 2008; Abomhara et al., 2010; Jian-Wen et al., 2006; Puri et al., 2004; Richardson, 2007-2011):

1. High Definition Digital TV

2. High Definition DVDs (HD-DVD and Blu-Ray formats)

3. Mobile TV broadcasting

4. Apple products including iTunes video downloads, iPod video and Mac OS

5. NATO and US DoD video applications

6. Internet video streaming applications

7. Videoconferencing

8. E-learning

But on the other hand, the complexity of encoding and decoding processes on H.264/AVC video compression standard was relative high, therefore H.264/AVC was not 
so suitable for real-time video streaming applications (Liu et al., 2009a; Wien et al., 2007).

\section{The H.264/AVC Extension for Scalable Video Coding (H.264/SVC)}

H.264/SVC as an extension for Scalable Video Coding to H.264/AVC standard was completed in the mid of 2007 (Wien et al., 2007). The term scalability refers to the exclusion of some parts of the video streaming's bits in order to deal with the numerous demands or preferences of end users as well as to variable terminal capabilities or network conditions (Schwarz et al., 2007). In fact H.264/SVC can efficiently handle spatial, temporal and quality (Signal-to-Noise Ratio (SNR)) scalabilities (Wien et al., 2007):

1) Spatial scalability: Is the resolution scalability of decoded video

2) Temporal scalability: Is the scalability standard that depends on the number of frames displayed per second (or we can call it frame rate scalability)

3) Quality scalability: Is the scalability standard that depends on the compression's level that applied to the source video during encoding process

In fact, the H.264/SVC can employ one or different combinations of these three scalabilities according to video communication scenario at hand (Wien et al., 2007). Therefore, the H.264/SVC can achieve effective video communication under different communication environments. So far there are three scalable profiles have been defined for H.264/SVC to deal with different video communication scenarios (Liu et al., 2009b; Schwarz et al., 2007):

1) Scalable Baseline profile: Was mainly used for conversational and surveillance applications that require a low decoding complexity

2) Scalable High profile: Was designed for broadcast, streaming and storage applications

3) Scalable High Intra profile: Was mainly designed for professional applications

In conclusion, the H.264/SVC compression standard achieves same visual reproduction quality as H.264/AVC with at most $10 \%$ higher bit rate (Liu et al., 2009a). However the H.264/SVC can achieve high coding efficiency in communication environment that require low complexity video compression algorithm by using low level scalabilities (like the Scalable Baseline profile or the low level of the Scalable High profile).

More specifically, in H.264/SVC, a less powerful decoder can extract fewer layers and decoding fewer layers means less decoding complexity than H.264/AVC. Furthermore, the scalabilities offered by the H.264/SVC can provide some extra functionalities like: Graceful degradation in lossy transmission environments as well as bit rate, format and power adaptation (Schwarz et al., 2007). Eventually, H.264/SVC achieves better RateDistortion (R-D) performance comparable with H.264/AVC (Liu et al., 2009a; Wien et al., 2007; Schwarz et al., 2007); Therefore, H.264/SVC are more suitable for real-time and live video streaming systems than the previous H.264/AVC standard (Liu et al., 2009a; Wien et al., 2007; Liu et al., 2009b).

\section{High Efficiency Video Coding (HEVC) Compression Standard}

HEVC is the latest compression standard, which was released in Jan. 2013 by the ITU-T Video Coding Experts Group (VCEG) and ISO/IEC Moving Picture Experts Group (MPEG). It outperforms all the previous compression standards (especially for a high-resolution video) (Sullivan et al., 2016). To be more specific, it leads to significant decrease in bit-rate by approximately $50 \%$ while keeping the same level of perceptual video quality as in the previous standards (Sullivan et al., 2016). Indeed, HEVC was designed to deal with high video resolution and high use of parallel processing architecture (Sullivan et al., 2016).

The following section highlights the video streaming error correction stage that follows the video compression stage.

Pitchaipillai and Eswaramoorthy (2017), this paper evaluates the performance of Link Reliable Energy Efficient Ad hoc On-demand Multipath Distance Vector (LREE- AOMDV) routing on H.264/MPEG-4 Advanced Video Coding (AVC) video streaming under both Distributed Coordination Function (DCF) and Enhanced Distributed Coordination Function (EDCF) using NS2.34 and enhanced my EvalVid framework.

Since the LR-EE-AOMDV routing protocol selects the link-reliable energy-efficient multiple routes based on minimal hop count, the end to end delay and average end to end delay of LR-EE-AOMDV are significantly reduced in comparison with OMMRE-AOMDV and AOMDV routing protocols under both DCA and EDCA in Video streaming environment.

\section{An Overview of the Errors Correction Techniques for Enhancing the Video Streaming Quality}

The error correction techniques are any methods used for detecting and correcting the errors. In this tutorial paper we present some important error correction techniques that used for enhancing the quality of Video Streaming over packet switched and best effort networks (e.g., the Internet). This section is organized as follows: In sub-section IV.A we show some crucial types of Forward Error Correction (FEC) techniques at the application layer and then in sub-section IV.B we present the most important Backward Error Correction (BEC) techniques at the application and transport layers. 


\section{Forward Error Correction (FEC) Techniques at the Application Layer}

The FEC techniques allow receiver to detect and fix errors (on the fly) over erasure channels. In other word the sender(in encoding process) should add redundant (or parity) bits to the source information symbols, then the receiver (in decoding process)can use these redundant bits to detect and fix the errors (as shown in Fig. 4) (Wang et al., 2002). As a matter of fact that the FEC techniques give receiver chance to correct errors without depending on a reverse channel to request data retransmission (or no need for feedbacks).

One of the well-known drawback of FEC techniques is that they cause more bandwidth overhead (or increase bits rate because of the redundant bits) than BEC techniques and hence results in reducing the amount of available bandwidth for the actual video streaming bits. But because of the fewer (or non) data retransmissions in FEC techniques, then they can correct the errors faster (or make less delay) than BEC techniques (Daintith, 2004), therefore the FEC techniques are more suitable for the real-time and live video streaming applications.

Here in this tutorial paper we focus on the following FEC techniques at the application layer:

\section{Reed-Solomon Codes}

Reed-Solomon code (Wicker and Bhargava, 1999) is cyclic error correction code, Also it relies on a method that detects and corrects multiple random errors in packet erasure channel by applying FEC technique; therefore we can say that Reed-Solomon is an erasure code.

Furthermore, Reed-Solomon code can generate encoded symbols $(n)$ (or a codeword $(n)$ ) from original source information symbols $(k)$ by over sampling primitive polynomial $p(x)$ at $n>k$ distinct points. Therefore Reed-Solomon code is $[n, k, n-k+1]$ code, or it is linear block code of length $(n)$, with dimension $(k)$ and at least it has $(n-k+1)$ Hamming distance. Also it can be said that the FEC encoder creates $(n-k)$ repair (or redundant) symbols in order to fix the errors (Mohr et al., 2000; Wicker and Bhargava, 1999; Hamzaoui et al., 2008; Akabri et al., 2007). However if the places of erroneous symbols are unknown in advance, then ReedSolomon code can fix up to $(n-k) / 2$ erroneous symbols.

As a matter of fact that the Reed-Solomon code is fixed (or static) rate code, because it relies on unchangeable code rate (code rate $=k / n$ ). Hence, smaller code rate (or $k / n$ ratio) leads to higher data protection, or in other words we can say that the larger data block length $(n)$ leads to higher data protection for a fixed value of $(k)$.

\section{Fountain Codes}

Fountain code (MacKay, 2005) is a type of erasure code but it is rateless code, or it does not rely on fixed code rate $(k / n)$. In the previous traditional erasure codes (like Reed-Solomon codes), the number of generated packets is fixed based on the best guess of network conditions prior to producing any packets and inevitably this guess is either too high or too low. But the fountain code can generate flexible number of encoded symbols $(n)$ from original source symbols $(k)$. Furthermore, fountain code is close to optimal for every erasure channel (MacKay, 2005). As a matter of fact that the Fountain code is based on the following one way FECsteps:

1) The sender encodes packets then sends them over the erasure channel

2) Then the receiver checks the encoded packets, if there is an error (and the error cannot be corrected) then the erroneous packet will be discarded (Hamzaoui et al., 2008). Else the packet will be kept as a part of the message

3) Finally if the receiver collects enough errorless packets to reconstruct the original message, then it sends signal to the receiver in order to finish the transmission

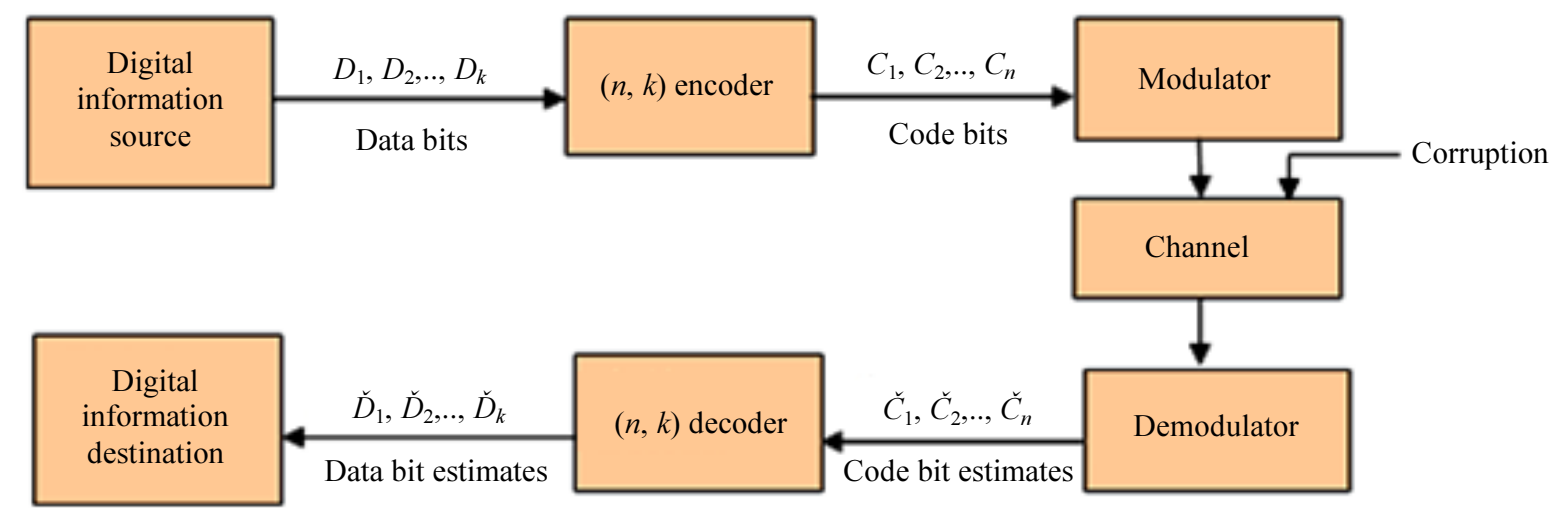

Fig. 4: General encoding and decoding processes in the FEC technique (Wang et al., 2002) 
As a matter of fact that fountain codes have the following advantages over the previous traditional erasure codes (like Reed-Solomon codes) (Ahmad et al., 2009):

1) In Fountain codes, the encoder (on the fly) can generate as few or as many encoded symbols as needed on demand, or we can say that the Fountain codes are more flexible than the previous traditional erasure codes

2) Fountain codes have faster encoding and decoding processes than the traditional erasure codes. Therefore the Fountain codes are suitable for the real-time video streaming over lossy packet networks.

The reliable decoding algorithm of length $(n)$ in fountain codes: Is an algorithm that can recover the all ( $k$ ) input symbols from any set of $(n)$ output symbols (Shokrollahi, 2003). Luby Transform (LT) and Raptor codes are the most famous classes of the Fountain codes and theses codes will be discussed in some more details by the next sub-sections.

\section{Luby Transform (LT) Codes}

LT code (MacKay, 2005; Luby, 2002) is the first practical realization class of erasure and rateless Fountain codes; Also LT code uses a simple algorithm that relies on eXclusive OR (XOR) operation for encoding and decoding (or errors detection) the message symbols.

As a matter of fact that LT codes have many advantages over the previous traditional erasure codes, for example the LT codes can generate close to the minimal number of encoded symbols $(n)$ and then sent them (in packets) to the receiver; Also the number of required packets for recovering the original data (in receiver side) is asymptotically close to the minimal possible. But on other hand LT codes have one drawback which is that LT codes are not systematic, this means that the input symbols (or original $(k)$ symbols) are not necessarily reproduced from the output symbols (or encoded (n) symbols) (Shokrollahi, 2003).

\section{Raptor Code}

Raptor code as demonstrated in (MacKay, 2005; Shokrollahi, 2003; Shokrollahi, 2006) is the first type of fountain codes with linear time algorithm for encoding and decoding. Also Raptor code has faster encoding and decoding processes than LT code (Hamzaoui et al., 2008), therefore Raptor code leads to further reduce the end to end delay. Furthermore, Raptor code can work in non-systematic and systematic ways, as many applications require systematic codes for better performance, in (Shokrollahi, 2003; Shokrollahi, 2006) they proposed a new set of ideas to design an efficient systematic version of Raptor code and this yields an invertible binary $k$ x $k$-matrix $R$ :

- To encode the input symbols, then the inverse of matrix $R$ should be used to transform these input symbols to intermediate symbols

- Then the matrix $R$ is used to transform these intermediate symbols back to the input symbols

Figure 5 shows a version of $\mathrm{f}$ Raptor codes that relies on multiple stages pre-condition:

1) The first stage is based on Hamming code encoding technique

2) The second stage is based on Low Density Parity Check (LDPC) coding (Pishro-Nik et al., 2005)

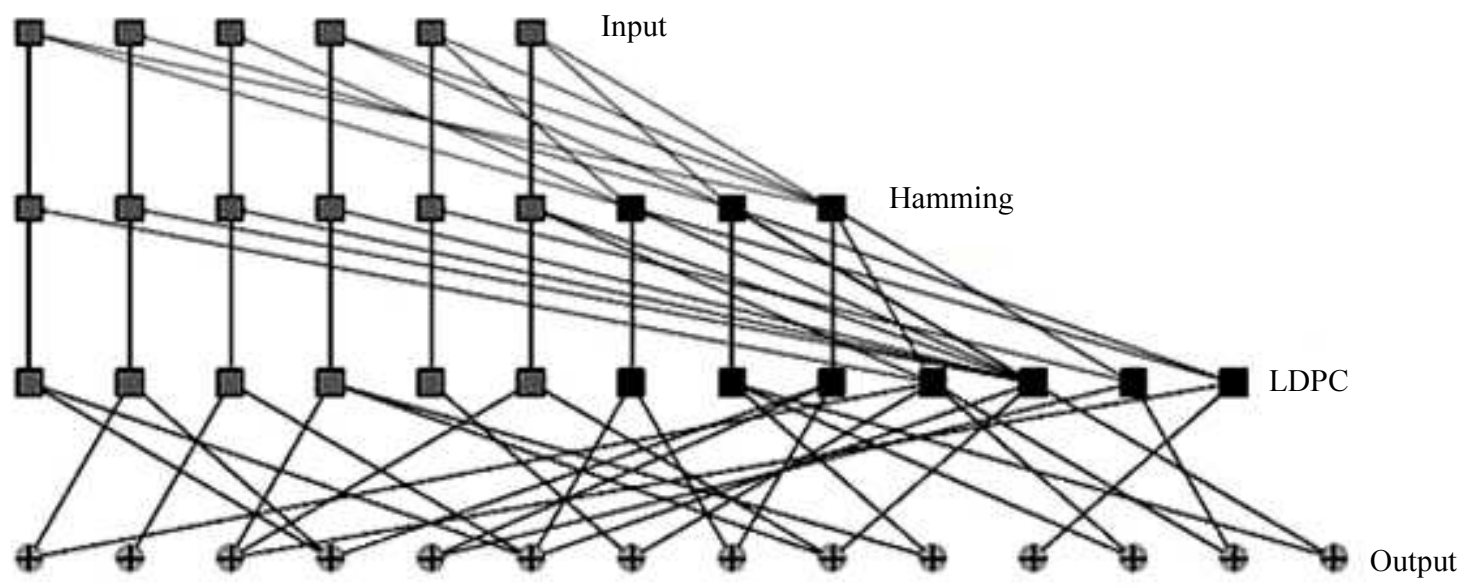

Fig. 5: One version of Raptor codes: The pre-condition is done in multiple stages. The first stage is based on Hamming code and the second stage is a version of LDPC coding (Courtesy of (Shokrollahi, 2003)) 


\section{Backward Error Correction (BEC) Techniques at} the Application and Transport Layers

$\mathrm{BEC}$ is an errors correction technique in which the receiver can discover the errors then it sends a request to the sender to retransmit the corrupted or lost data (or packets) again (Elaywe and Kamal, 2002), In other words BEC requires reverse (or feedback) channel to inform the sender about the corrupted or lost data, As a matter of fact that there are two methods to inform the sender about the corrupted data:

1) Positive Acknowledgment (ACK) method: Here the receiver sends an acknowledgement message for every correctly received data (or packet) and then the sender will only retransmit the not acknowledged data (or packets) within an appropriate time (as shown in Fig. 6)

2) Negative Acknowledgment (NACK) method: Here the receiver only sends a request to the sender in order to retransmit the not acknowledged (or corrupted) data (as shown in Fig. 7)

A well-known disadvantage of the BEC is that it leads to increase the average delay in the data receiving process and hence the BEC is not suitable for the live video streaming applications. Here in this tutorial paper we focus on the following BEC techniques at the application and transport layers:

\section{Transmission Control Protocol (TCP)}

TCP is a high reliable general purpose flow control protocol, therefore the packets should arrive to the destination in the same order they are sent without any loss or error, Furthermore TCP relies on lost packets retransmission technique and this retransmission was based on either duplicated ACK messages or Timeout (Elaywe and Kamal, 2002). But on the other hand TCP is inappropriate for real-time video streaming applications (typically loss-tolerant and delay sensitive) for two reasons:

1) TCP is too slow (or leads to high round trip delays) since reliability is obtained from repeated packets retransmission (Elaywe and Kamal, 2002), Therefore these long delays lead to violating the real-time requirement of video streaming (Ghareeb, 2008)

2) Also TCP uses an end-to-end congestion control mechanism which is based on Linear Increase Multiplicative Decrease (LIMD) philosophy. In case there are no loss packets then the source's transmission rate is linearly increased. But on other hand if there is some loss packets then its transmission rate is decreased by a large factor (multiplicative decrease) (Puri et al., 1997)

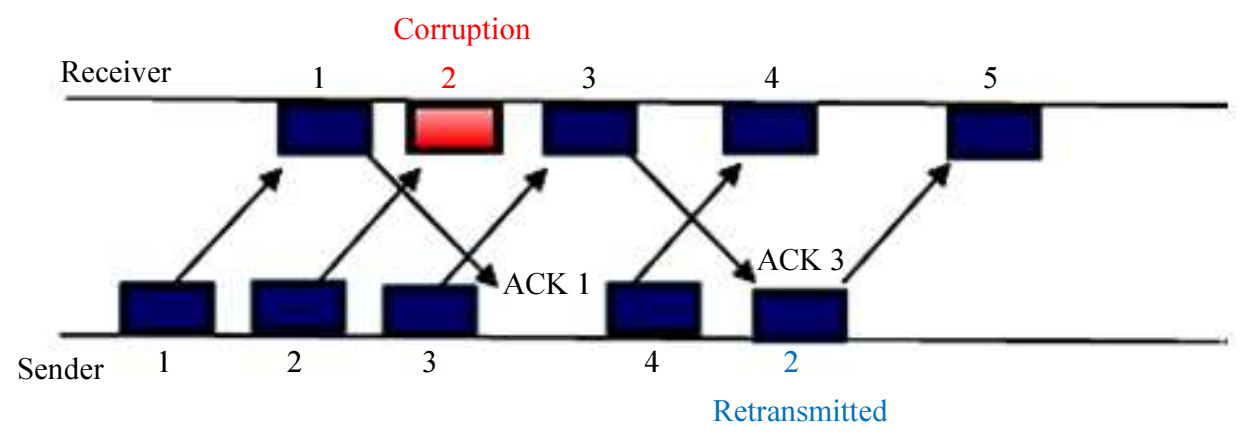

Fig. 6: BEC with positive Acknowledgment (ACK)

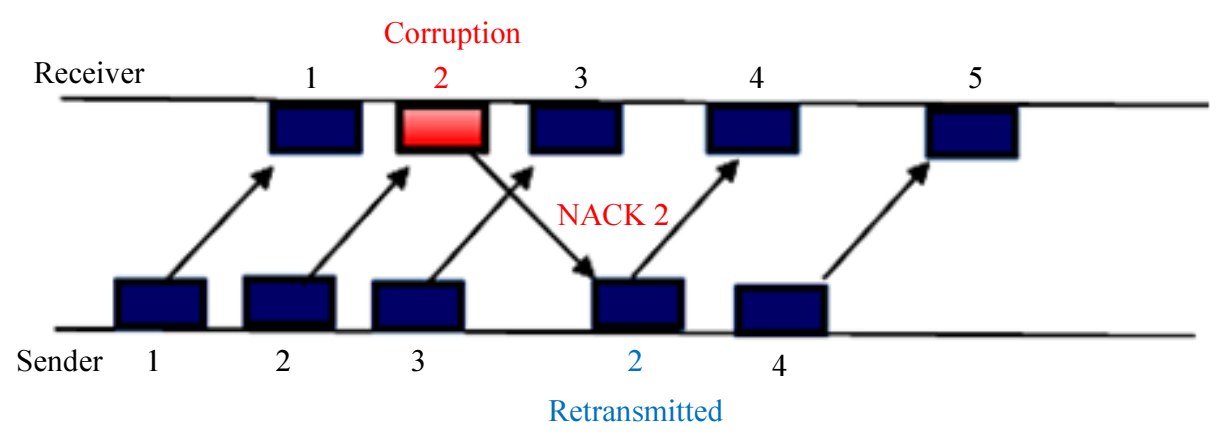

Fig. 7: BEC with negative Acknowledgment (NACK) 


\section{TCP-Friendly Rate Control Protocol (TFRC)}

In TFRC (Mahdavi and Floyd, 1997) the senders should measure loss packets and delay in the network and then they set up the transmission rate according to the following equation:

$$
B=\frac{1.3 * M T U}{R T T * \sqrt{\operatorname{loss}}}
$$

where, $(B)$ is the Bandwidth; Maximum Transmission Unit (MTU) is the maximum packet size being used on the connection; RTT is the Round Trip Time and Loss is the packets loss rate. In this technique they did not use the TCP congestion control and packets retransmission techniques; However in (Mahdavi and Floyd, 1997) they used the TCP congestion control's history (like the round trip delay and packets loss rate) to only calculate the bandwidth over the User Datagram Protocol (UDP) (or best effort protocol). As a matter of fact that TFRC is suitable for the continuous media flows and video streaming applications because it can provide a very smooth rate control which can be wellmatched to the multimedia applications (Puri et al., 1997; Mahdavi and Floyd, 1997; Sisalem and Schulzrinne, 1998; Padhye et al., 1999). However TFRC is not suitable for the live video streaming for the following reasons (Puri et al., 1997):

1) TFRC is slow to respond to network dynamic circumstances (like sudden changes in the congestion level)

2) Also TFRC's success depends on accurate measurements of the Round Trip Delay and the packet loss probability, the latter is very difficult to calibrate accurately in a responsive manner since it requires large observation intervals

\section{Reliable UDP (RUDP)}

UDP transport protocol is suitable for real-time application, But on other hand it is not reliable and doesn't rely on any sequencing, lost packets retransmission and congestion control techniques. In other words, UDP is loss-tolerant and delay sensitive, Therefore it increases the lost packets rates and hence reduces the video streaming quality (e.g., decrease the PSNR) (Al-Akaidi and Hamzaoui, 2011; Ghareeb, 2008).

However, RUDP is a type of UDP protocols, but it tries to improve the UDP reliability and performance, Therefore RUDP takes some features from the TCP protocol, For example, RUDP tries to add some general purpose congestion control mechanism, acknowledgement and some lost packets retransmission in order to enhance the performance and reliability of UDP protocol (Bova and Krivoruchka, 1999).

\section{Reliable Blast UDP (RBUDP)}

RBUDP is a special type of reliable datagram protocols, which intended for handling high speed bulk data transfer (Al-Akaidi and Hamzaoui, 2011). Also, we can say that the RBUDP is designed for high bandwidth, dedicated- or Quality-of-Service- enabled networks. Furthermore, the experiments in ( $\mathrm{He}$ et al., 2002) showed that the RBUDP performs extremely efficiently over high speed dedicated networks.

\section{Real-time Transport Protocol (RTP)}

RTP (Sisalem and Schulzrinne, 1998) supported UDP with extra features to deal with the real-time video streaming and continuous media applications over the Internet; In fact, these features can be applied to make differentiation between various media streams; Also these features help in keeping track of different statistics based on the transmission quality as observed by other members of the session. Furthermore, they supported RTP with functionalities for estimating the bottleneck bandwidth of a connection based on the packet pair approach (Sisalem and Schulzrinne, 1998; Schulzrinne and Casner, 1996). Eventually, RTP provided time stamping, sequence numbering and other techniques to deal with the timing problems (Ghareeb, 2008).

\section{Bidirectional Transport Protocol (BTP)}

BTP is a special purpose and advanced version of the UDP protocol, As a matter of fact that BTP is intended for the tele-controlled robot applications; Furthermore BTP relies on inter-packet gap (IPG) congestion control technique (Al-Akaidi and Hamzaoui, 2011).

\section{Embedded Reliable Transport Protocol (ERT)}

In ERT, they added an extra header to the existing UDP segment in order to enhance the reliability for embedded system applications (Al-Akaidi and Hamzaoui, 2011).

\section{Datagram Congestion Control Protocol (DCCP)}

DCCP is an unreliable general purpose transport protocol, but on the other side it has some congestion control mechanisms (e.g., TFRC-Like). However, the performance of DCCP over WiMAX networks is better than the performance of UDP. Furthermore, the multi-connection DCCP can be successfully used for the real-time video streaming over WiMAX networks (Al-Akaidi and Hamzaoui, 2011).

\section{Broadband Video Streaming (BVS)}

BVS is a semi-reliable special purpose transport protocol that used for broadband video streaming applications, Furthermore, BVS applies retransmissions only for the lost prioritized packets by implementing the Quick Response (QR) technique (Al-Akaidi and 
Hamzaoui, 2011). Hint, in QR technique the receiver should send NACK for each lost packet.

\section{Original Random Early Detection (RED)}

The Random Early Detection (RED) method is a router-based approach to detect the onset of congestion to sources to maintain the network in a region of low delay and high throughput, therefore RED is designed to avoid congestion rather than react to it (Floyd and Jacobson, 1993; Kamal, 2004).

RED should be able to control the average FIFO queue size (in the router) and therefore control the average delay. In case if the average queue size is less than the minimum threshold (THmin) then the router will add the packet to the queue, but if the average queue size is between minimum threshold (THmin) and maximum threshold (THmax) then router will discard the packet with drop probability $(\mathrm{P})$, else the router will add the packet to the queue with probability (1-P), Finally the router will definitely discard the packet if the average queue size is over the maximum threshold (THmax) Fig. 8 and 9. Therefore RED may reduce the traffic injection rate before congestion happens.

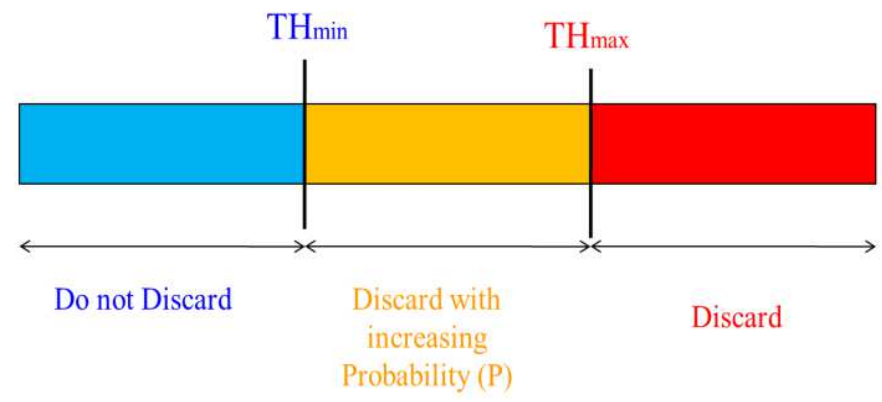

Fig. 8: The three cases of the router average queue size (Floyd and Jacobson, 1993)

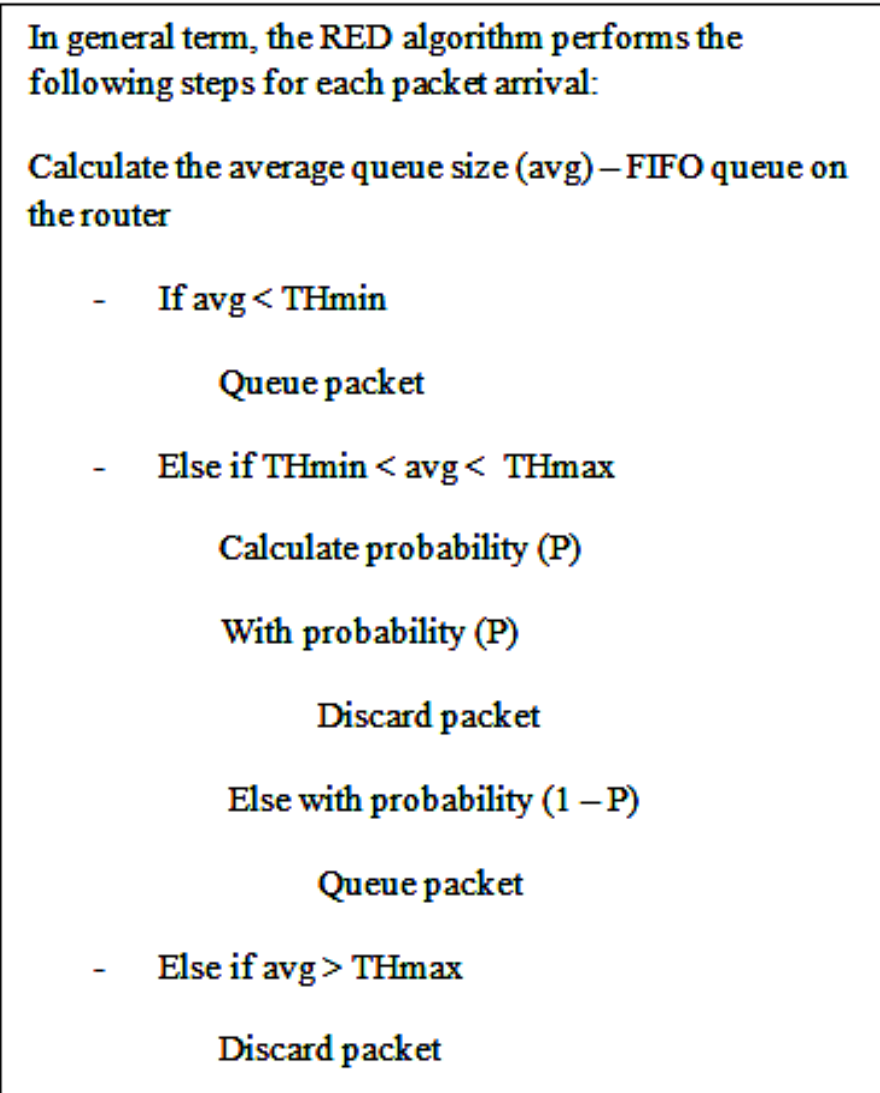

Fig. 9: General algorithm for RED routers 
Whereas the RED algorithm was good for centralized (or infrastructure) networks, But as a result of:

1) Congestion may simultaneously affect multiple queues in different routers in Ad-hoc networks

2) Also the queue at a single node cannot completely reflect the state of a certain Ad-hoc network

Then the original RED failed to improve the fairness in the Ad-hoc networks (Xu et al., 2005).

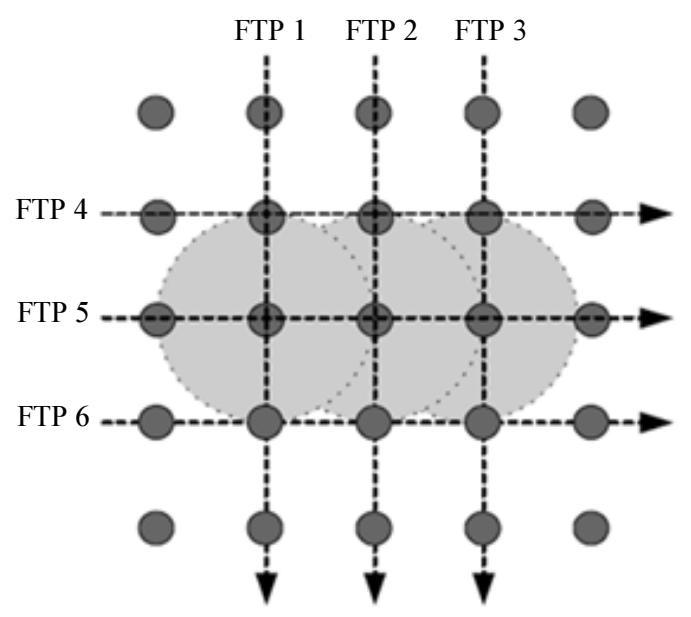

Fig. 10: Scenario for the multiple congested neighborhood topology (Courtesy of (Xu et al., 2005))

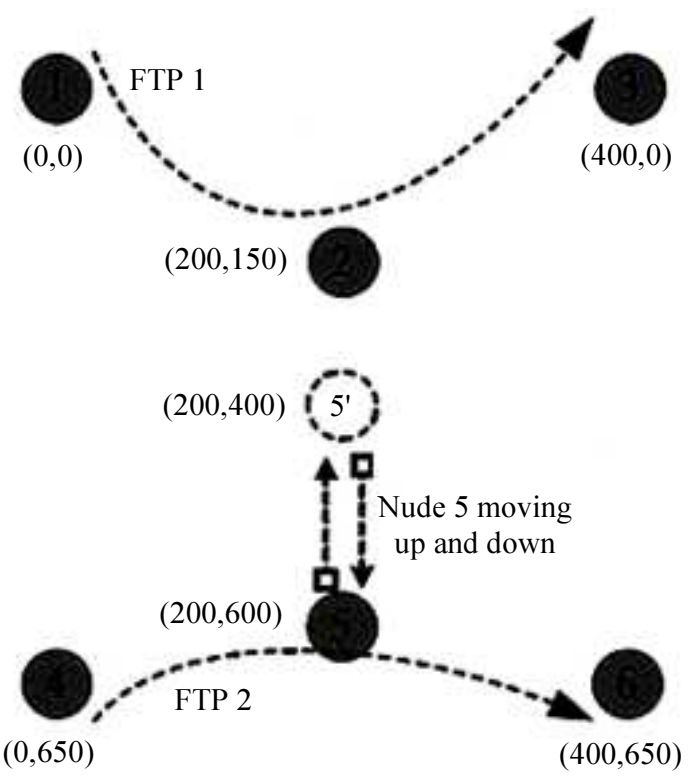

Fig. 11: Scenario for evaluating NRED under mobility (Courtesy of (Xu et al., 2005))

\section{Neighborhood RED (NRED) Solution}

In (Xu et al., 2005; 2003), they proposed a good solution to solve the unfairness problem in the Ad-hoc networks. They supposed that each node should broadcast a Neighborhood Congestion Notification (NCN) packet to inform neighbors about its congestion status. The NCN packet must contain enough information for a neighbor node to calculate its local drop probability $(\mathrm{P})$. Therefore this solution extends the RED concept to the distributed neighborhood queue. Furthermore some Simulation studies proved that the NRED scheme can improve the TCP fairness in ad hoc networks (Fig. 10 and 11).

Santos-González et al. (2017) proposes two new mobile applications that implement those protocols in Android whose objective is to know how they are influenced by the aspects that most affect the streaming quality of service, which are the connection establishment time and the stream reception time. The experimental results of the analysis show that the developed WebRTC implementation improves the performance of the most popular video streaming applications with respect to the stream packet delay.

The use of the WebRTC protocol provides better QoE and QoS than other protocols and that the implemented Direct WebRTC system offers good results,

\section{Enhancing the Video Streaming Quality by Implementing Forward Error Correction (FEC) Codes with Feedbacks}

In this section, we highlight some of the previous works on enhancing the video streaming quality by applying the Forwarded Error Corrections (FEC) techniques (in sub-section IV.A) with some feedbacks over packet switched networks. As a matter of fact FEC techniques are faster and more suitable for live and realtime video streaming applications over packet erasure channels than BEC techniques.

In sub-section V.A we show an integrated ReedSolomon FEC source transcoding and updated TFRC congestion control protocol for enhancing video streaming in the Internet. Then in sub-section V.B we talk about an Integration of Reed-Solomon FEC coding and updated TFRC congestion control with Receiver-Driven Protocol for enhancing the quality of distributed video streaming. In sub-section V.C we show a compound solution of Reed-Solomon FEC coding and packets retransmission BEC technique for enhancing the quality of Peer-to-Peer video streaming. Finally, in sub-section V.D we present an adaptive live unicast video streaming with rateless fountain codes and feedback. 
An Integrated Reed-Solomon FEC Source Transcoding and Updated TFRC Congestion Control Protocol for Enhancing Video Streaming in the Internet

In (Puri et al., 1997) they proposed an integrated solution between Reed-Solomon FEC source trans coding and an updated TFRC congestion control technique (called Linear Increase Multiplicative Decrease with History or $\mathrm{LIMD} / \mathrm{H}$ protocol) for enhancing the quality of video streaming in the Internet (as shown in Fig. 12). Furthermore in (Puri et al., 1997) they depended on the following techniques:

1) Multi Resolution (MR) Video Source coding: Is a layered representation (for more details about layered representation you can refer to section VII) of the coded bit video stream (as shown in Fig. 13). Some of the multimedia compression standards (e.g., Motion Picture Experts Group-4 (MPEG-4)) are evolving towards a multi-resolution (MR)

2) Multiple Description FEC Video Coding (MDFEC): In (Puri et al., 1997), they supposed that the Reed-Solomon FEC coding was depended on Multiple Description (MD) concept (as shown in Fig. 14). Actually, MD provides the property of higher number of received packets leads to better received video streaming quality (Puri et al., 1997; Sisalem and Schulzrinne, 1998). Furthermore, an adaptive rate Reed-Solomon MD-FEC type was applied in (Puri et al., 1997). Also the ReedSolomon MD-FEC relies on the congestion feedbacks from the receiver (that is in the form of the number of packets successfully received in a particular time period) to guess the channel state and set the new code rate

3) LIMD/H (or updated TFRC) Congestion Control protocol: In (Puri et al., 1997) they used LIMD/H (or updated TFRC protocol in sub-section IV. B. ii)to calculate the adaptive bandwidth over the packet-switched networks. Also they used the feedbacks from the receiver to make the bandwidth calculation more accurate and to have better responding to network dynamic circumstances (like sudden changes in congestion level). LIMD/H has the following advantages:

a) $\mathrm{LIMD} / \mathrm{H}$ uses the history of packet losses for distinguishing between congestion-induced and non-congestion-induced packet losses

b) LIMD/H deals gently with the non congestioninduced packet losses in order to keep the sending rate variation to minimum, but it deals quickly to the onset of the congestion

c) In the steady state it delivers the same average rate as any TCP based algorithm would do and hence is TCP friendly

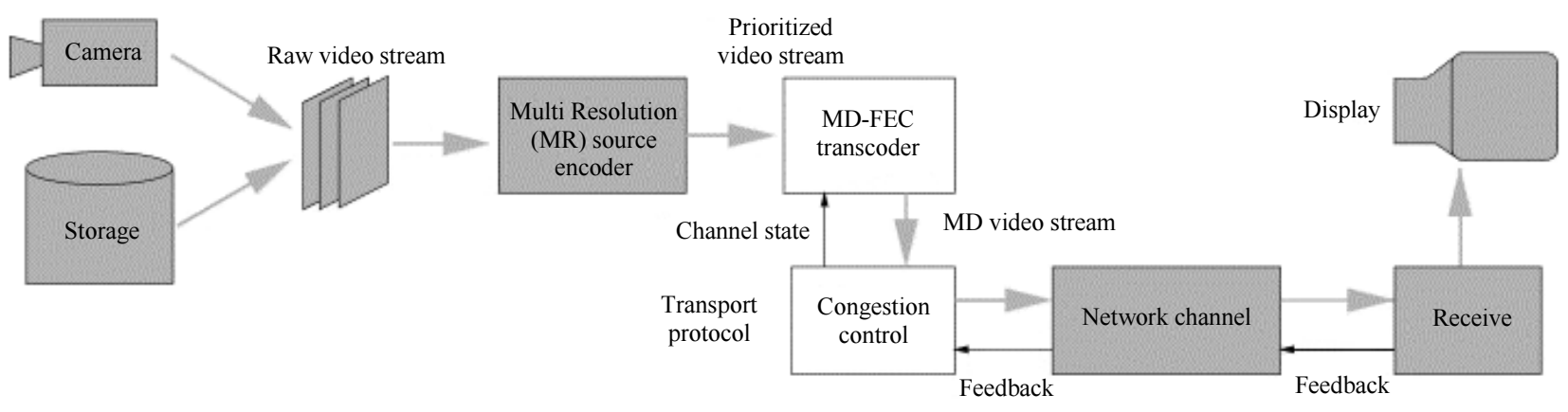

Fig. 12: Block diagram of end-to-end internet video streaming transmission system (Courtesy of (Puri et al., 1997))

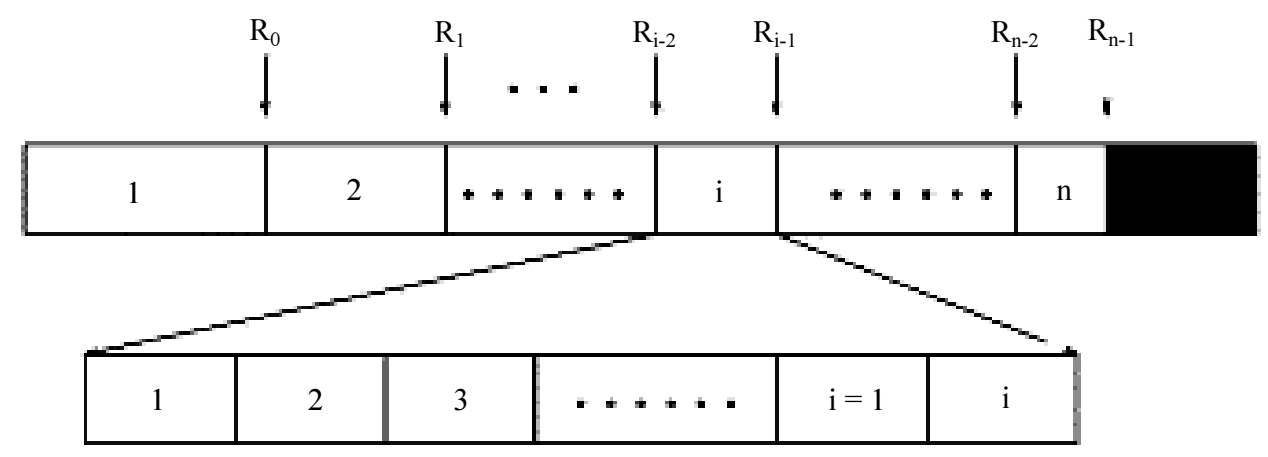

Fig. 13: Progressive bit stream from the source coder partitioned into N layers or quality levels (Courtesy of (Puri et al., 1997)) 


\begin{tabular}{|c|c|c|c|c|c|c|}
\hline Section 1 & Section 2 & $\cdots$ & Section $i$ & $\cdots$ & Section $N$ & \multirow[b]{2}{*}{ Description 1} \\
\hline 1 & 2 & $\cdots$ & $i$ & $\cdots$ & $N$ & \\
\hline \multicolumn{7}{|c|}{ 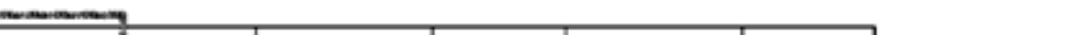 } \\
\hline FEC & 2 & $\cdots$ & $i$ & $\cdots$ & $N$ & Description 2 \\
\hline \multicolumn{6}{|c|}{$:$} & $\dot{:}$ \\
\hline FEC & FEC & $\cdots$ & $i$ & $\cdots$ & $N$ & \multirow[t]{2}{*}{ Description $i$} \\
\hline & & & 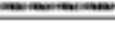 & & & \\
\hline \multirow[t]{3}{*}{ FEC } & FEC & $\cdots$ & FEC & $\cdots$ & $N$ & Description $i+1$ \\
\hline & \multirow{2}{*}{\multicolumn{3}{|c|}{$\vdots$}} & \multirow{2}{*}{\multicolumn{2}{|c|}{$t$}} & $\vdots$ \\
\hline & & & & & & $\cdot$ \\
\hline FEC & FEC & $\cdots$ & FEC & $\cdots$ & $N$ & Description $N$ \\
\hline
\end{tabular}

Fig. 14: N-description generalized MD codes using forward error correction codes (Courtesy of (Puri et al., 1997))

In conclusion, the integration between Reed-Solomon MD-FEC and LIMD/H proposed in (Puri et al., 1997) has good impacts on enhancing the quality of video streaming over the Internet. But on other hand the Reed-Solomon MD-FEC and LIMD/H still suffer from bandwidth overhead problem and can't deal very well with packets loss and delay issues in dynamic (or unknown circumstances) packet erasure channels. Therefore, the proposed solution in (Puri et al., 1997) was not suitable for real-time and live video streaming applications.

An Integration of Reed-Solomon FEC Coding and Updated TFRC Congestion Control with ReceiverDriven Protocol for Enhancing the Quality of Distributed Video Streaming

In (Nguyen and Zakhor, 2002) they presented an integrated solution for enhancing the quality of video streaming quality in simple distributed network that consisted of three senders and one receiver (as shown in Fig. 15) and hence (Nguyen and Zakhor, 2002) leads to high throughput and increasing the tolerance for packet loss and delay due to network congestion. Actually in (Nguyen and Zakhor, 2002), they make integration between Receiver-Driven protocol and Reed-Solomon FEC coding:

1) Receiver-Driven protocol: In (Nguyen and Zakhor, 2002), the receiver can organize the transmissions from the three senders based on the information (like the round trip delays) that received from the three senders. The proposed Receiver-Driven protocol consisted of (a) updated receiver TFRC rate allocation protocol and (b) packets allocation algorithm:

a) The Updated Receiver TFRC Rate Allocation Protocol: In (Nguyen and Zakhor, 2002) they proposed that the receiver can determine the sending rate for each sender based on an updated TFRC protocol (refer to sub-section IV.B.ii for more details).Therefore the receiver should send control information (or packet) to the sender (as shown in Fig. 15c) and hence the sender can use these control information to calculate the available bandwidth based on the following equation:

$$
B=\frac{s}{R \sqrt{\frac{2 P}{3}} \operatorname{Trto}\left(3 \sqrt{\frac{2 P}{3}}\right) p\left(1+32 p^{2}\right)}
$$

where, $(B)$ is the current available bandwidth between each sender and the receiver, $\left(T_{r t o}\right)$ is TCP time out, $(R)$ is the estimated Round Trip Time in seconds, $(P)$ is the estimated loss rate and $(s)$ is the TCP segment size in bytes

b) The Packets Allocation Algorithm: It ensures that no sender can send the same packets to the receiver at the same time (by depending on the control packet from the receiver) and hence minimizes the probability of late packets at the receiver

2) Reed-Solomon FEC coding: In (Nguyen and Zakhor, 2002) they used a traditional fixed (or static) rate Reed-Solomon coding technique for enhancing the visual quality of the streamed video and hence reduces the lost packets in low delay multimedia communications as well as streaming applications on the Internet

However, the traditional fixed-rate Reed-Solomon FEC codes and TFRC still have some bandwidth overhead problems and can't deal very well with packets loss and delay issues in dynamic (or unknown circumstances) packet erasure channels. Therefore, the method presented in (Nguyen and Zakhor, 2002) also was not suitable for realtime and live video streaming applications. 


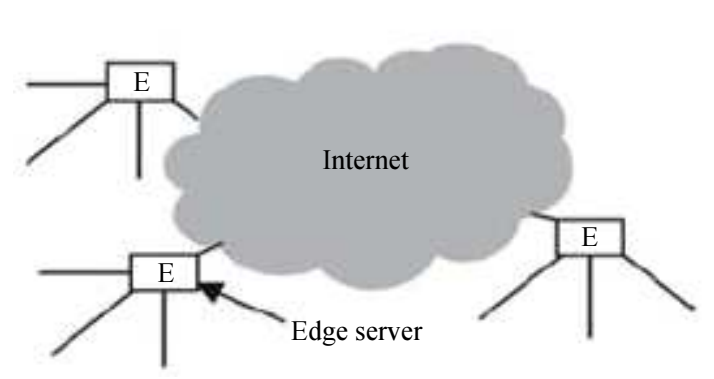

(a)

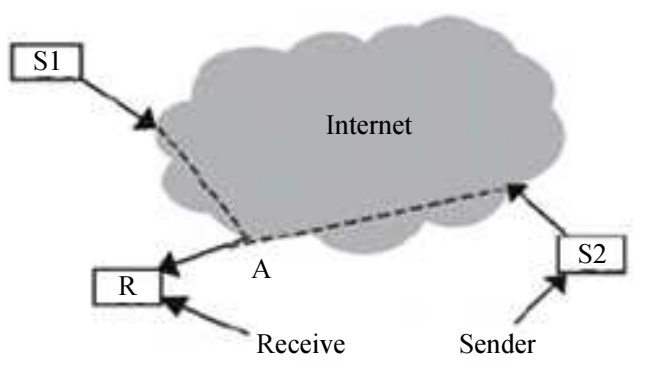

(b)

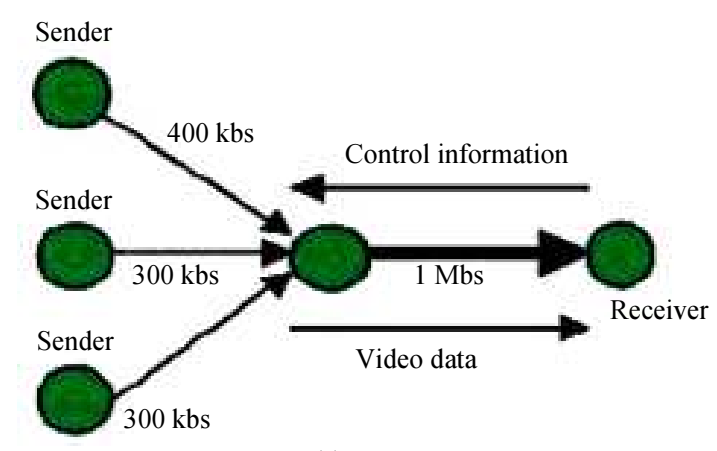

(c)

Fig. 15: (a) Edge server architecture; (b) Distributed video streaming architecture; (c) Distributed video streaming (Courtesy of (Nguyen and Zakhor, 2002))

Compound Solution of Reed-Solomon Coding and Packets Retransmission Techniques for Enhancing the Quality of Peer-to-Peer Video Streaming

In Peer-to-Peer (P2P) video streaming each party can act as a client or as a server during the communication (Sukanen, 2002). By the way in (Akabri et al., 2007) they propose a compound solution of Reed-Solomon FEC Coding and packets retransmission BEC technique for enhancing the quality of P2P video streaming, while MPEG-4 was used as a video streaming compression standard. In this compound solution, they proposed two scenarios:

1) In the first scenario: They supposed that just I-frames are protected by using Reed-Solomon FEC Coding, whereas P- and B-frames are recovered by some retransmission BEC technique with feedback channel

2) In the second scenario: They supposed that both I- and P-frames are protected by using Reed-Solomon FEC Coding and B-frames are recovered by some retransmission BEC technique with feedback channel

Furthermore, in (Akabri et al., 2007) they concluded that these compound scenarios' achieved less bandwidth overhead than the full Reed-Solomon FEC solution. Also, they reduced the packets loss rate and delay with comparison to full retransmission BEC technique with feedback channel.
On the other hand, as mentioned before that the fixed-rate Reed-Solomon FEC coding still suffer from bandwidth overhead problems, also retransmission BEC technique with feedback channel can't deal very well with packets loss and delay issues over dynamic packet erasure channels. Therefore, the compound solution presented in (Akabri et al., 2007) was also not suitable for live and real-time video streaming applications.

However, in next sub-section we present a better technique that depend on Rateless Fountain FEC Codes and Feedback to further decreasing the FEC coding bandwidth overhead while keeping a good reconstruction of the video streaming and hence results in enhancing the average PSNR and decreasing the congestion level over packet erasure channels with unknown circumstances.

\section{Adaptive Live Unicast Video Streaming with Rateless Fountain Codes and Feedback}

According to the previous sub-sections it can be concluded that the traditional fixed (or static) - rate FEC code (like Reed-Solomon code) can't deal with delay and lost packets issues in dynamic (or unknown circumstances) packet erasure channels (like the Internet and wireless networks). Furthermore, Fig. 16 shows that applying the fixed (or static) - rate FEC code leads to a big mismatch between the actual packets loss rate and the predicted packets loss rate. To be more specific, overestimating the packet loss rate leads to wasting the 
bandwidth, although underestimating it results in decoding failure (Ahmad et al., 2010).

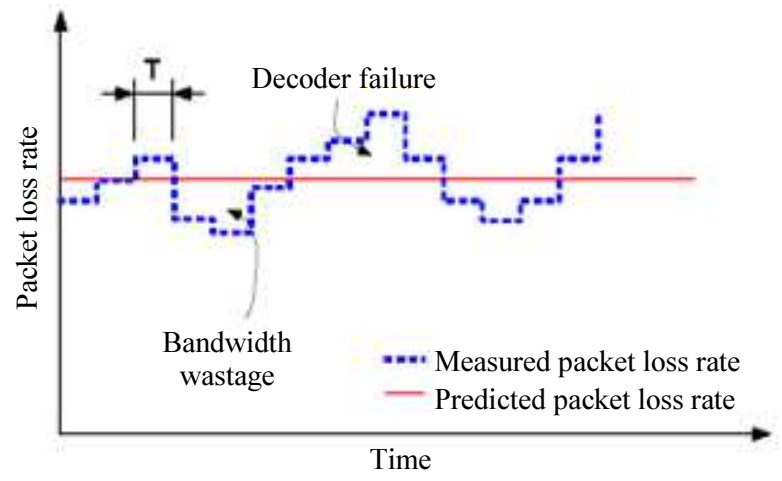

(a)

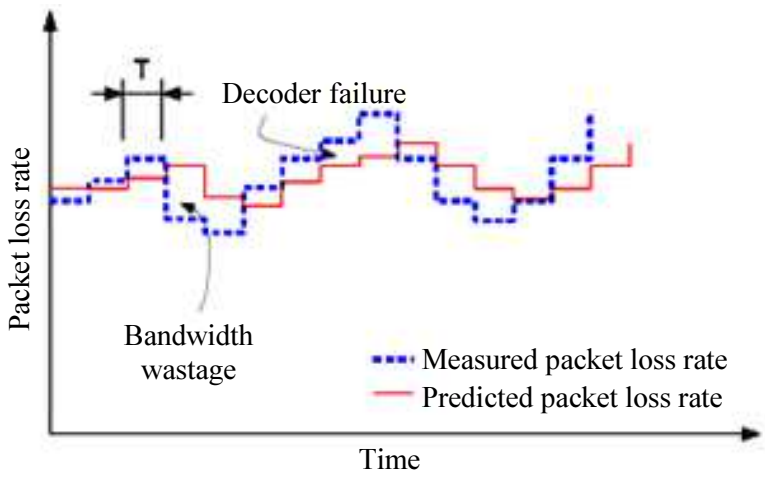

(b)

Fig. 16: Difference between observed and predicted packet loss rate. The observed packet loss rate corresponds to transmission intervals of length T. (a) Static prediction. (b) Adaptive prediction (Courtesy of (Ahmad et al., 2007))

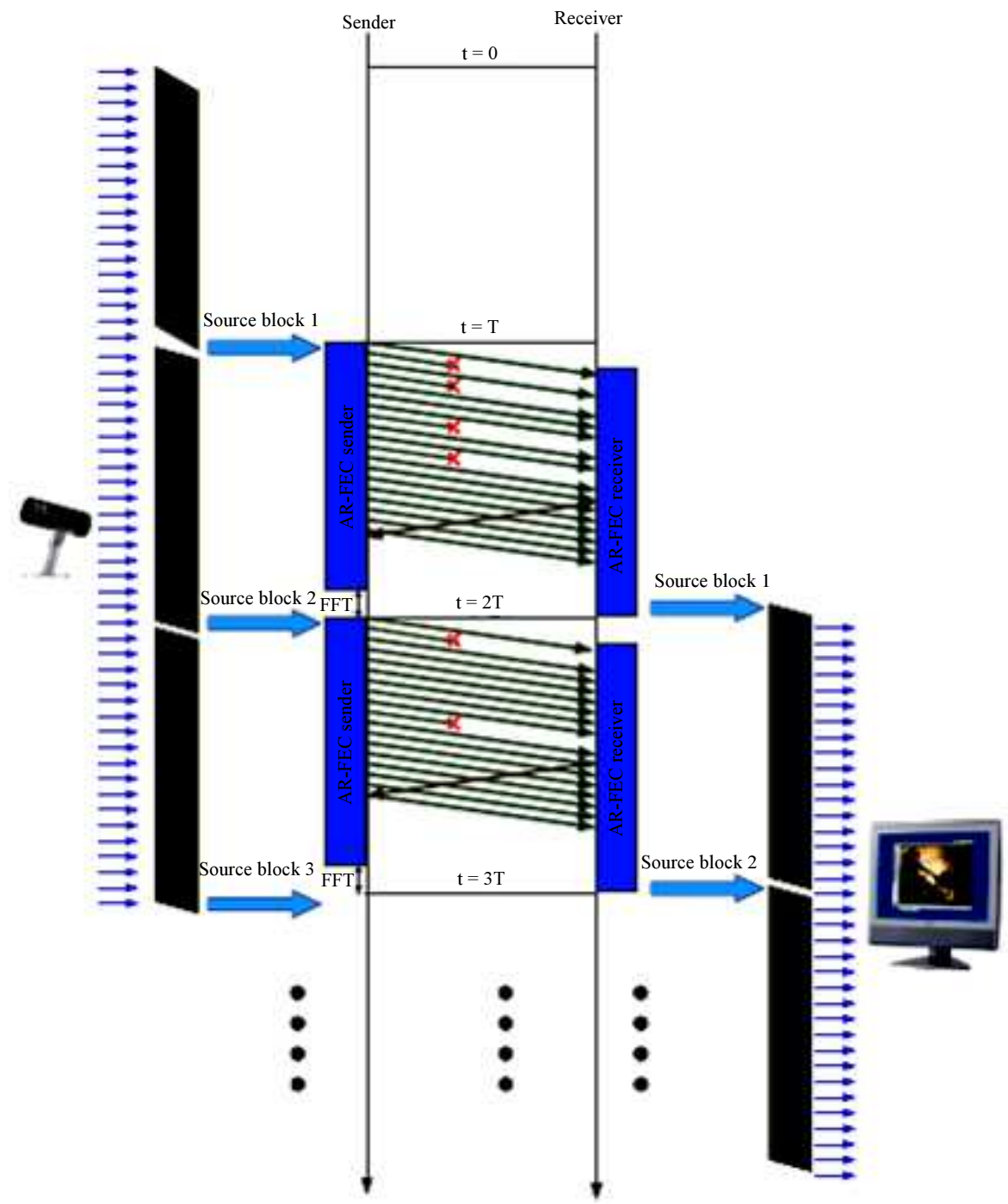

Fig. 17: One scenario for live video streaming system: This video streaming consisted of (b) blocks and each block consisted of (k) source information symbols. (Courtesy of (Ahmad et al., 2007)) 
To address this problem, in (Ahmad et al., 2007) they applied fast rateless Raptor FEC code on the live unicast video streaming application over packet erasure networks. Also they supposed that there is a reliable (no lost packets or delays) feedback channel, then the receiver can send an acknowledgment (or feedback) to the sender as soon as sufficient encoded symbols (to reconstruct the targeted source block) are received (as shown in Fig. 17), Therefore these feedbacks can help in updating the existed packets loss rate histogram (as shown in Fig. 18). Furthermore, the code rate $(\mathrm{k} / \mathrm{n})$ and as a result transmission rate (as shown in Fig. 19) are flexible and depend on the last observations (or prior histogram as shown in Fig. 18) of the packets loss rates. Therefore, the packet loss rate histogram was employed to optimize the transmission strategy over packet erasure networks. Also this flexible transmission strategy (as showed in Fig. 19) was consisted of a sequence of alternating transmission and waiting periods. Indeed, these waiting periods lead to reducing the bandwidth overhead (Fig. 20) while ensuring successful decoding subject to an upper bound on the packet loss rate (Ahmad et al., 2007).

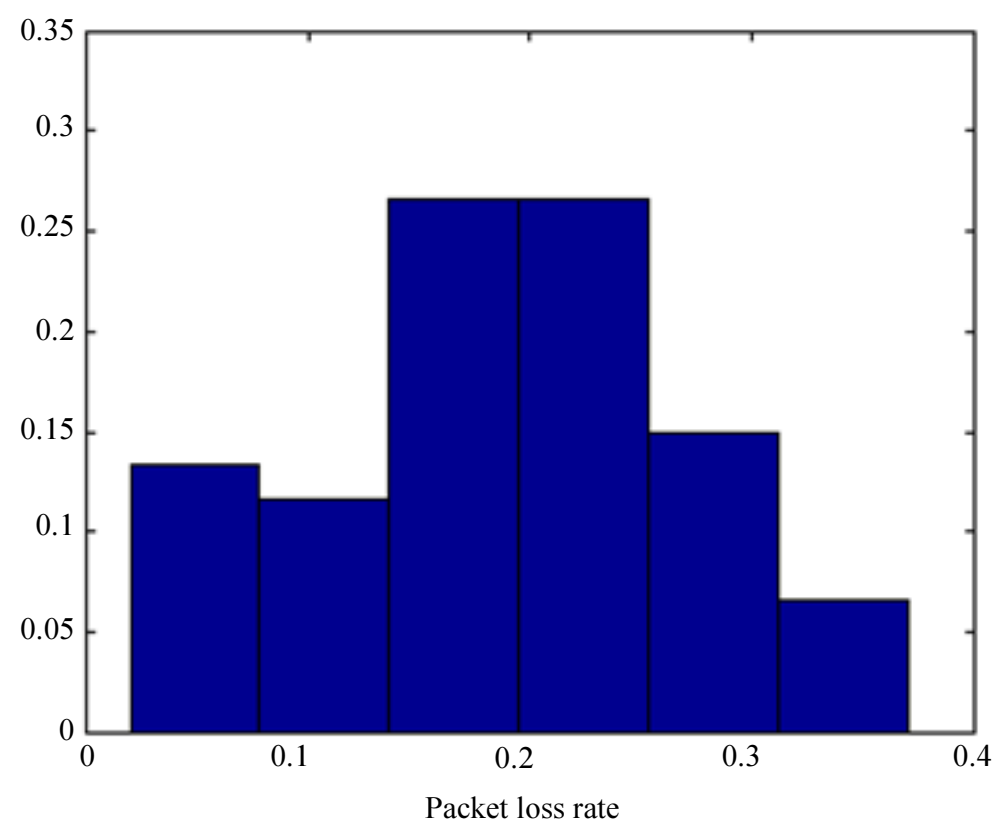

Fig. 18: The packet loss rate histogram with 6 bins for the link Konstanz-Beijing-Konstanz. (Courtesy of Ahmad et al., 2008a))

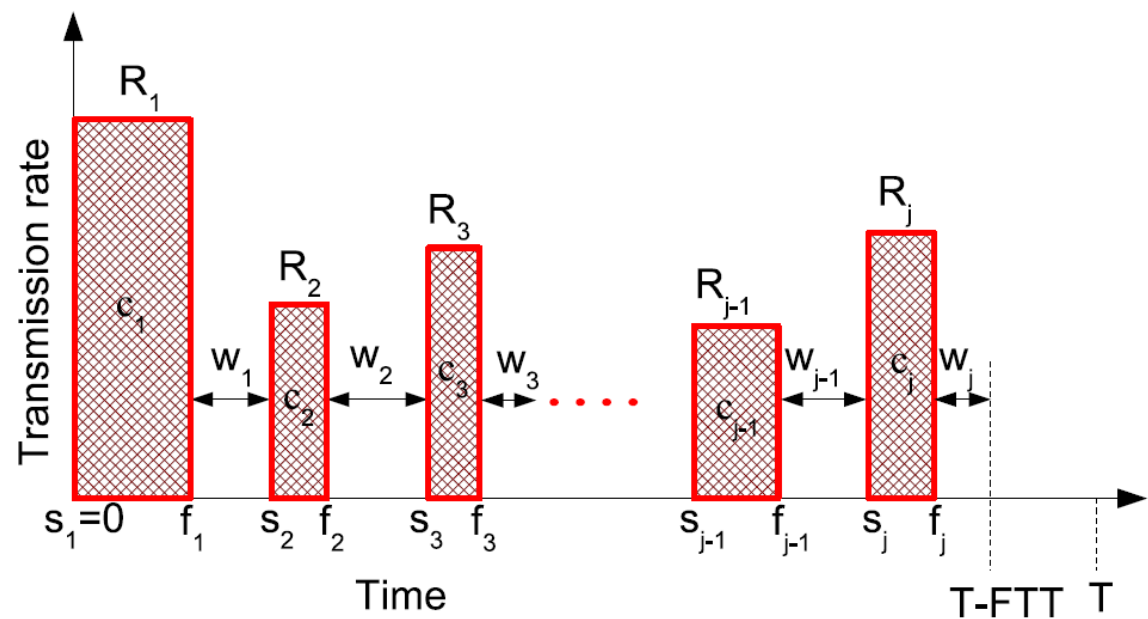

Fig. 19: The flexible transmission strategy. Whereas the encoded symbols are transmitted at rate $R_{i}$ from $s_{i}$ to $f_{i}$, followed by a waiting time of $\mathrm{w}_{\mathrm{i}}, \mathrm{i}=1, \ldots, \mathrm{j}$. (Courtesy of (Ahmad et al., 2007)) 


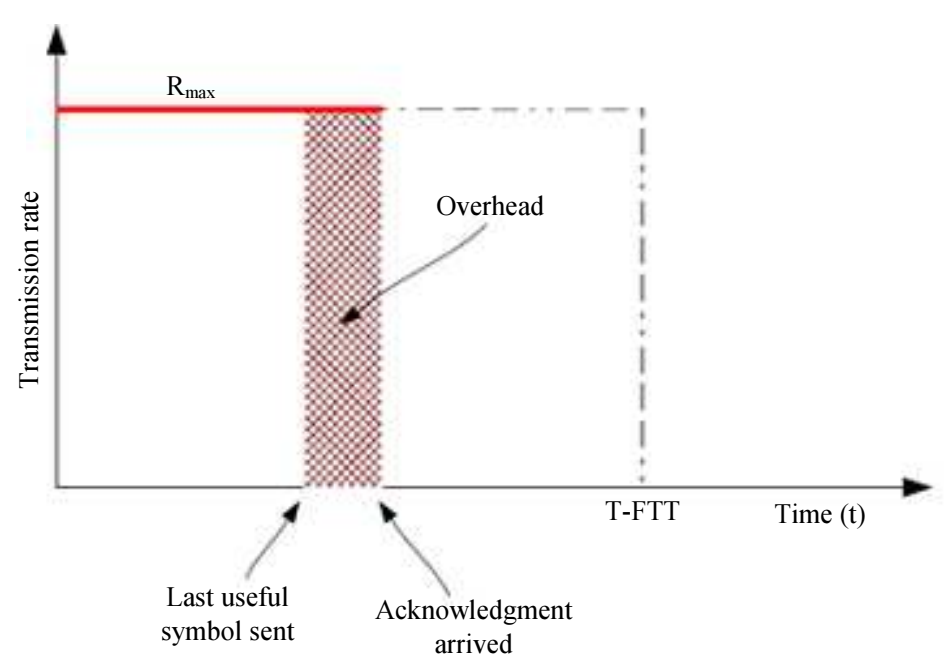

(a)

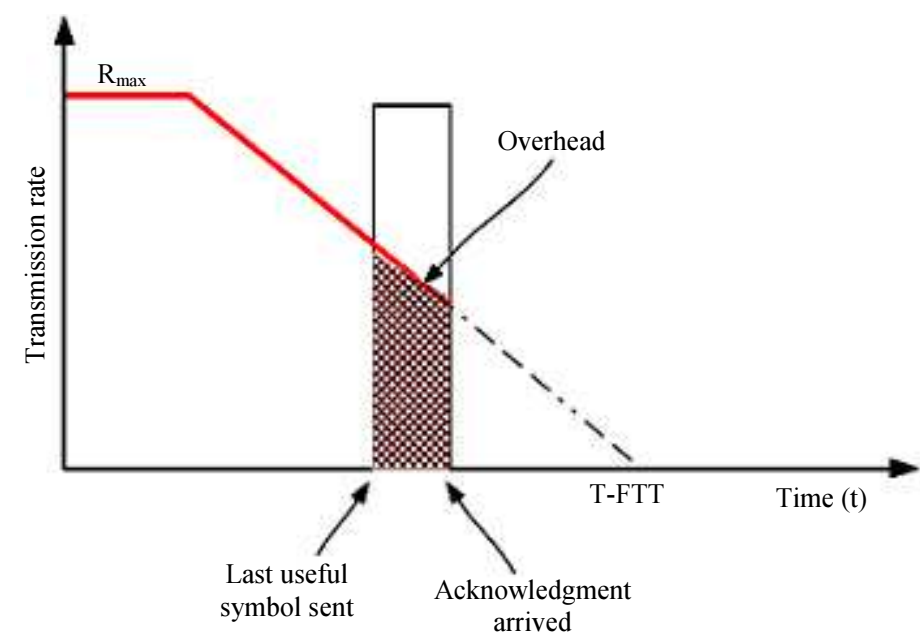

(b)

Fig. 20: (a) Fixed transmission rate strategy Vs. (b) Flexible transmission rate strategy. Whereas the shaded areas show the overhead. (Courtesy of (Ahmad et al., 2007))

Ahmad et al. (2008a), they introduced a practical implementation of (Ahmad et al., 2007), also they supposed the following:

1) The feedback channel is not reliable enough.

2) Also the packet may contain more than one symbol.

3) Finally they supposed that the packets loss rate histogram did not exist before the beginning of transmission (as shown in Fig. 18), therefore one can start with an arbitrary packet loss rate histogram then this histogram is updated (by the sender) continuously in real-time based on the feedback (or acknowledgment) about the number of received packets from the receiver.

Furthermore in (Ahmad et al., 2008a), they implemented (Ahmad et al., 2007) and tested the performance of the live unicast video streaming system under H.264/AVC video compression standard. Then they make a comparison (based on Peak Signal to Noise Ratio (PSNR)) between the following schemes:

a) Algorithm 1: Proposed in (Ahmad et al., 2007) and based on prior packet loss rate histogram

b) Algorithm 2: Practical version of Algorithm 1 (Ahmad et al., 2008a), also the packet loss rate histogram is computed in real-time

c) Static: Fixed rate code until acknowledgement come from the receiver

d) Adaptive: Here the code rate is selected depending on the lost packets rate that observed in the previous source block transmission interval only (not based on the packet loss rate histogram)

e) Without FEC: This scheme did not use FEC 


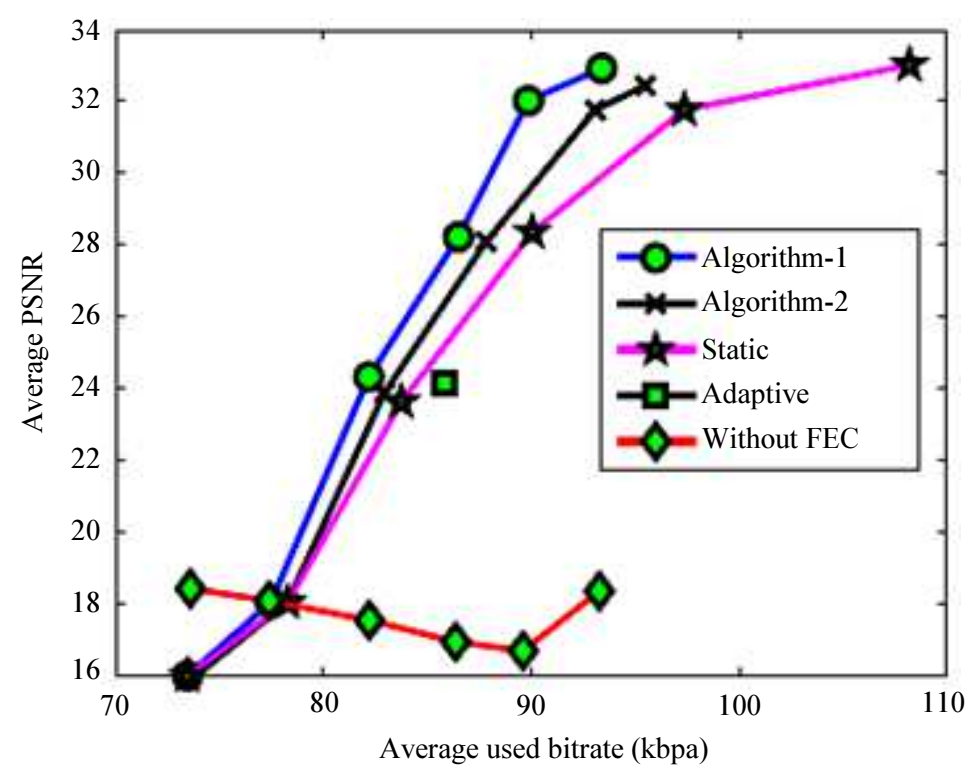

Fig. 21: Average PSNR (in dB) vs. average used bandwidth (in kbps). (Courtesy of (Ahmad et al., 2008a))

Actually, Fig. 21 shows that schemes (a) and (b) achieved better PSNR than (c),(d) and (e).However, scheme (b) had a slightly worse performance than scheme (a) and this because it did not rely on prior observations (or histogram) of the packets loss rates (Ahmad et al., 2008a). For instance (as shown in Fig. 21), at $89.86 \mathrm{kbps}$, whereas scheme (a) achieved an average PSNR of $32.02 \mathrm{~dB}$, scheme (c) reached an average PSNR of $28.36 \mathrm{~dB}$ at $90.04 \mathrm{kbps}$ (Ahmad et al., 2008a). Furthermore, scheme (c) achieved better PSNR than (d) and this is because the packet loss rate is rapidly changing, so as a result the packet loss rate prediction from only the previous source block interval (not from the histogram) will be not efficient (Ahmad et al., 2008a).

In addition, the transmission strategies and packet loss rate histogram (as shown in Fig. 18) in (Ahmad et al., 2007; 2008a) lead to decreasing the FEC coding bandwidth overhead while keeping good reconstruction of the video streaming and hence results in enhancing the average PSNR and decreasing the congestion level.

Ahmad et al. (2010) they did a lot of experiments to show that the performance of solutions in (Ahmad et al., $2007 ; 2008$ a) is close to optimal compared to other FEC solutions for live or prerecorded video streaming over packet erasure channel. Also in (Ahmad et al., 2010) they proved that depending on a packet loss rates histogram (in (Ahmad et al., 2007; 2008a) achieves better adaptation to unknown channel circumstances and unpredictable packet loss rates. Furthermore, they supposed that there is an optimistic guess (send packets and wait for acknowledgement):

- In case if the sender did not receive an acknowledgment from the receiver, then this means that the guess was wrong, so the sender should make the next optimistic guess (excluding the previous one) and repeat the same procedure

- However, the delayed or lost acknowledgement feedback will have a bad effect on the system performance, Therefore if the packet loss rate is close to 1 (as in the worst case scenario), then the system performance will be similar to fixed (or static) -rate coding technique

Finally, (Ahmad et al., 2010) shows that the system performance can be enhanced in case if they increased the number of bins in the packet loss histogram. However if the number of bins increased to more than 6 bins then it increases the time complexity of the algorithm without any significant effects on the system performance.

In next section we concentrate on applying some FEC codes with UEP techniques in order to further enhancing the live and real-time video streaming quality over packet erasure networks.

\section{Enhancing the Video Streaming Quality via Using Forward Error Correction (FEC) Codes with Unequal Loss (or Error) Protection Techniques}

The Unequal Loss (or Error) Protection (ULP or UEP) technique is one of the powerful techniques for enhancing the quality of live and real-time video streaming over packet erasure networks.

In this section, we present some of the previous works on enhancing the video streaming quality by implementing the ULP or UEP techniques over packet erasure networks. Actually, in this section we supposed 
that the transmitted data are vary in importance, for example, if we want to transmit a person face's image, then the data that allow us to recognize the person are more important than the data that show the texture of a few strands of hair. So if the packet erasure network is unable to transmit all of the data (because there are some lost packets), then we would like it to discard the part describing the hair and retain the part that allows recognition of the face's image. Therefore, the ULP or UEP techniques keep the most important data without loss (or error), while these techniques ignore the least important data. On other hand, the EEP techniques deal equally with all the data, whether they are most important or least important data (Mohr et al., 2000; Pishro-Nik et al., 2005; Rahnavard and Fekri, 2006; Rahnavard et al., 2007a).

Furthermore, in this section we present some UEP techniques that based on some Forward Error Correction (FEC) codes, therefore, the lost (or error) data will not be retransmitted again (or there is no feedback channel).

For simplicity, in this section we start with some techniques for enhancing just one single still image quality over packet erasure channels via using UEP with Reed-Solomon Forward Error Correction codes in subsection VI.A. Then in sub-section VI.B we present the results of applying the Unequal Error Protection (UEP) with rateless FEC code on MPEG video compression standards. In sub-section VI.C, we will shift to more advanced UEP techniques with Luby Transform (LT) codes to enhance the quality of unicast and multicast video streaming over packet erasure channels. Finally, in sub-section VI.D we show the adaptive UEP technique which leads to significant improvements in terms of average PSNR over previous static UEP techniques.

Unequal Loss Protection Technique for Graceful Degradation of Single Still Image Quality over Packet Erasure Channels through Reed-Solomon Forward Error Correction Codes

For simplicity, let us now start with enhancing the quality of transmitting just one single still image over packet erasure channels via using ULP technique, then in sub-section VI.B and sub-section VI.C we will shift to more advanced ULP techniques that are responsible for enhancing the quality of video streaming.

In (Mohr et al., 2000), they proposed an ULP technique that adds unequal amounts of Reed-Solomon FEC codes to the one single still image that transmitted over packet erasure and without feedback networks. Furthermore, in (Mohr et al., 2000) they considered the effect of each data byte on still image quality when assigning the FEC protection level (or priority level).

As a matter of fact that in (Mohr et al., 2000), the Reed-Solomon FEC codes are added to each message part in order to protect these parts against the packet loss, such that each message part and the FEC construct a stream, by the way Fig. 22 shows one possible method to transmit a message of 32 bytes of data (numbers 1-32) and 10 bytes of FEC (F).

Notice that in Fig. 22 above, more bytes of FEC are added to the first parts of the message whereas fewer bytes are added to the last parts of the message and this is because that the first parts of the message are most important to the overall quality of the reproduction, or we can say that the first parts of the message have the highest priority (Mohr et al., 2000).

Figure 23 shows that ULP in (Mohr et al., 2000) achieved graceful degradation of the single still image quality when the image transmitted over packet erasure networks with packet loss rates of $10 \%, 20 \%, 30 \%$ and $40 \%$, notice that the image quality keep high at a $30 \%$ packet loss rate, furthermore the image is still clearly recognizable even at $40 \%$ packet loss rate.

However, as we demonstrated in sub-section IV.A.ii that the Reed-Solomon fixed (or static) -rate FEC codes have the following disadvantages (Ahmad et al., 2009):

1) In Reed-Solomon fixed-rate codes, the encoder (on the fly) can't generate as few or as many encoded symbols as needed on demand, or we can say that the Reed-Solomon codes are less flexible than the rateless Fountain erasure codes

2) Also the Reed-Solomon fixed-rate codes have slower encoding and decoding processes than the rateless Fountain erasure codes

Therefore, the ULP techniques that depend on ReedSolomon fixed-rate FEC codes are not suitable for transmitting live and real-time video streaming over packet erasure networks with dynamic and unpredictable circumstances (like sudden changes in the congestion level).

However, in next sub-sections we present more advanced UEP techniques that depend on Rateless Fountain FEC Codes and will help in enhancing the quality (like the PSNR) of real time and live video streaming over packet erasure networks.

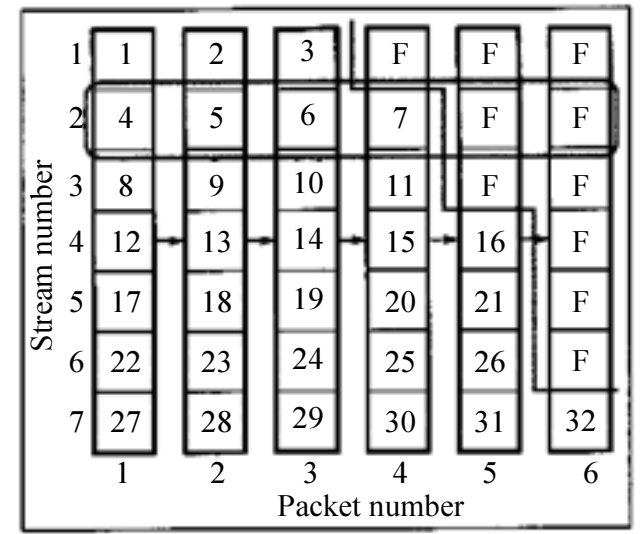

Fig. 22: Each of the rows is a stream and each of the columns is a packet. A stream contains 1 byte from each packet. The numbers $1-32$ are data and the symbol $\mathrm{F}$ is FEC (Courtesy of (Mohr et al., 2000)) 


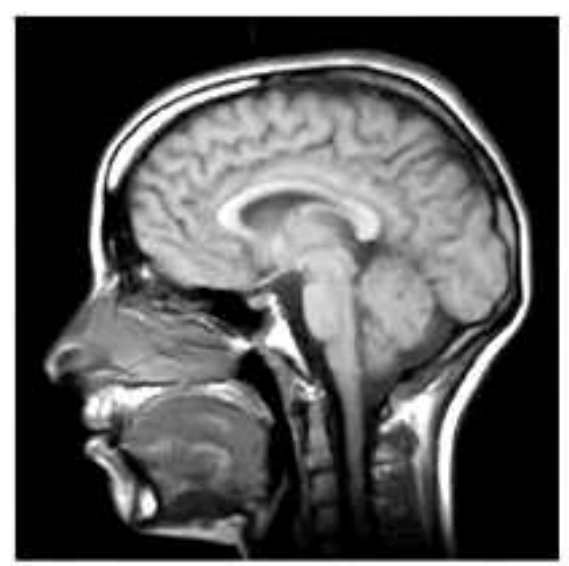

(a)

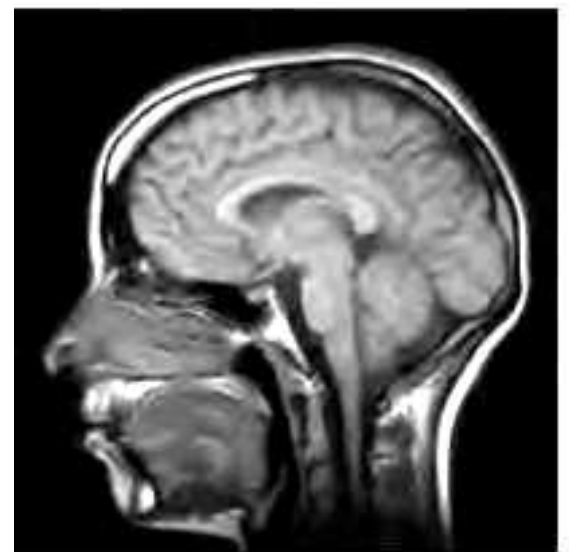

(c)

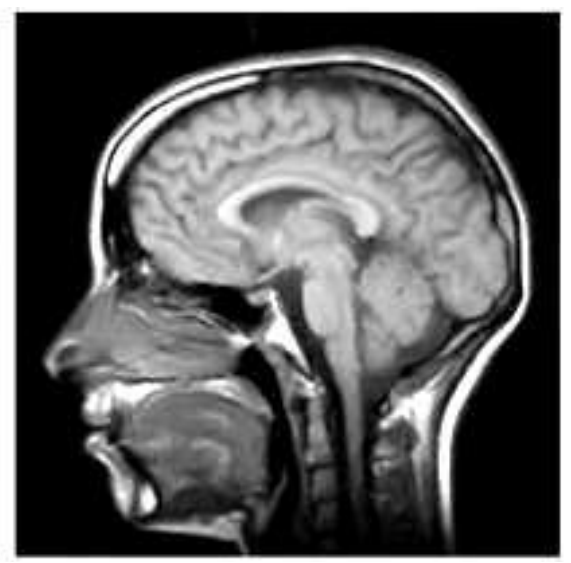

(b)

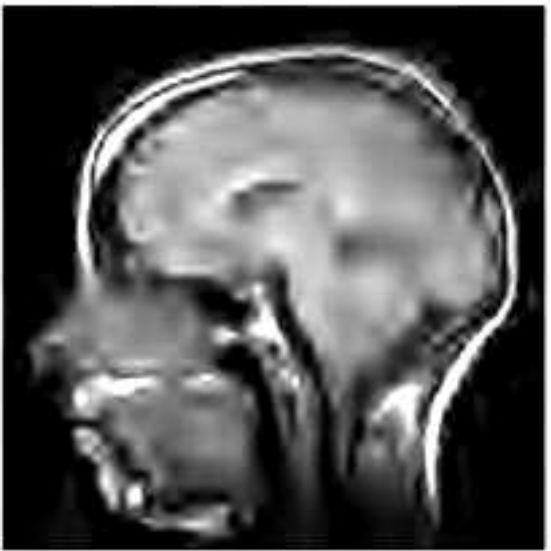

(d)

Fig. 23: Image quality at 1.0 bit per pixel total rate for Unequal Loss Protection of a magnetic resonance image over a channel that has an exponential loss profile with a mean of $10 \%$. (a) $10 \%$ of packets lost. (b) $20 \%$ of packets lost. (c) $30 \%$ of packets lost. (d) $40 \%$ of packets lost (Courtesy of (Mohr et al., 2000))

Information symbol

Encoded symbol

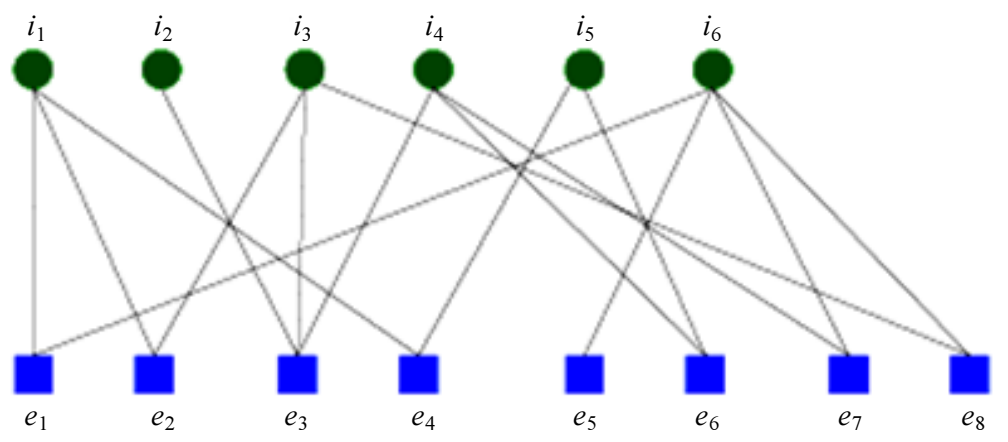

Fig. 24: Encoding graph of an LT code. Eight encoded symbols are generated from $\mathrm{k}=6$ information symbols. The degree of an encoded symbol is the number of information symbols that were used to generate it. For example, the degree of e1 is equal to two (Courtesy of (Ahmad et al., 2009)) 
Information symbol

Encoded symbol

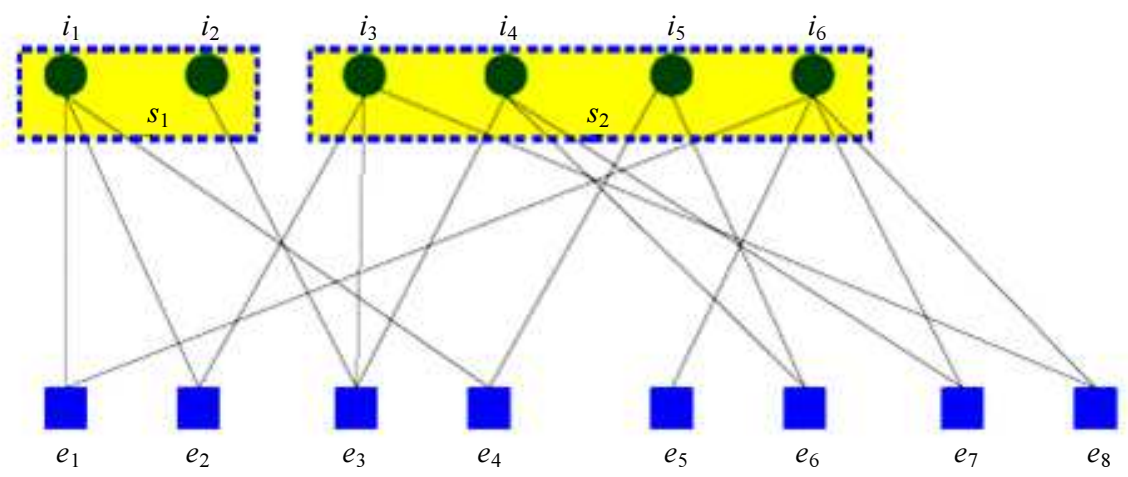

Fig. 25: UEP scheme 1(proposed in (Rahnavard et al., 2007b)): Two levels of protection are used. The set of MIB contains two information symbols while the set of LIB contains four information symbols (Courtesy of (Ahmad et al., 2009))

Applying Unequal Error Protection (UEP) with Rateless FEC Coding to MPEG Video Compression standards

The live and real-time video streaming quality can be further enhanced by using Unequal Error Protection (UEP) techniques with rateless FEC codes. In UEP techniques the information symbols protection are categorized into two main classes of errors protection (Rahnavard and Fekri, 2006; Rahnavard et al., 2007a): (as shown in Fig. 24 and 25):

a) Least Important Bits (LIB) symbols protection

b) Most Important Bits (MIB) symbols protection

Indeed, the probability of selecting LIB symbols is less than the probability of selecting MIB symbols. Therefore, the Bits Error Rate (BER) in LIB symbols is more than BER in MIB symbols (Fig. 25), notice that BER is computed as the average value of $(k-d) / k$, where (d) is the number of (correctly) decoded symbols at the receiver end (Ahmad et al., 2009).

Talari and Rahnavard (2009), they employed the UEP scheme 1 of (Rahnavard et al., 2007b) (as shown in Fig. 25) with rateless FEC coding (Shokrollahi, 2006) to encode MPEG-1 and MPEG-2 video frames over wireless packet erasure networks. Also in (Talari and Rahnavard, 2009), they supposed that different frames (like: I-Frames, P-Frames and B-Frames) have different network protection levels according to their importance, since decoding of $\mathrm{P}$ - and B-frames depends on the availability of the preceding I- and P-frames, therefore, I-frames are more important than $\mathrm{P}$-frames and $\mathrm{P}$-frames are more important than B-frames, then as a result of this order, I- frames should have the highest protection level, then Pframes should have medium protection level and finally B-frames should have the least protection level.

From an error control view, they in (Talari and Rahnavard, 2009) selected decodable frame rate (Q) to evaluate the quality of the decoded video since it can be mathematically expressed and it closely reflects the behavior of PSNR:

$Q=\frac{E[\text { aRnumber of decoded frames }]}{\text { Total number of transmitted frames }}$

Furthermore, in (Talari and Rahnavard, 2009) they proved that using UEP scheme 1 (Rahnavard et al., 2007b) with rateless FEC coding leads to increasing $Q$ and consequently the PSNR of the MPEG-1 and MPEG2 video (at the receiver end) compared to video coding with EEP rateless codes. To be more specific, in MPEG2 the decodable frame rate (Q) was increased from $58 \%$ (in EEP rateless code) to $74 \%$ (in UEP scheme 1 (Rahnavard et al., 2007b) with rateless FEC coding).

However, next sub-section presents more advanced Unequal Error Protection with Rateless Luby Transform (LT) Codes for H.264/SVC compression standard over packet erasure channels.

Enhancing the Unicast and Multicast Video Streaming via using the recent static Unequal

\section{Error Protection with Rateless Luby Transform (LT) Codes}

In (Ahmad et al., 2009; 2008b), they proposed a better and simpler static UEP scheme with Rateless 
Luby Transform (LT) codes (as shown in Fig. 26) for the scalable video coding (H.264/SVC) compression standard (see sub-section III.G), also they supposed the following:

1) The original source block consisted of (k) source information symbols and these symbols can be divided into two sets of information symbols2 MIB symbols and 4 LIB symbols. The information symbols in $\mathrm{I}$ and $\mathrm{P}$ frames can be assigned as MIB symbols, whereas the information symbols in B can be assigned as LIB symbols

2) Also the Repeat Factor (RF) for MIB symbols $=2$

3) The Expand Factor (EF) for (MIB and LIB) symbols $=2$
4) Finally the Original Source Block of 2 MIB symbols and 4LIB symbols can be transformed to a Virtual Source Bock of $(\mathrm{EF} \times(\mathrm{RF} \times 2+4))=(2 \times(2 \times 2+4))=$ $(2 \times 8)=16$ symbols

Then the LT-FEC encoder takes these virtual source block information symbols (in bits or bytes) and generates a potentially infinite sequence of encoded symbols (Ahmad et al., 2011).

Furthermore, in (Ahmad et al., 2009) they used the Recent static UEP scheme (Fig. 27) with LT rateless codes over multicast video streaming applications with heterogeneous receivers (for more details about the multicast video streaming you can refer to sub-section VIII.B.iii); Also they (Ahmad et al., 2009) make a comparison between: The previous UEP scheme lof (Rahnavard et al., 2007b) (Fig. 25),

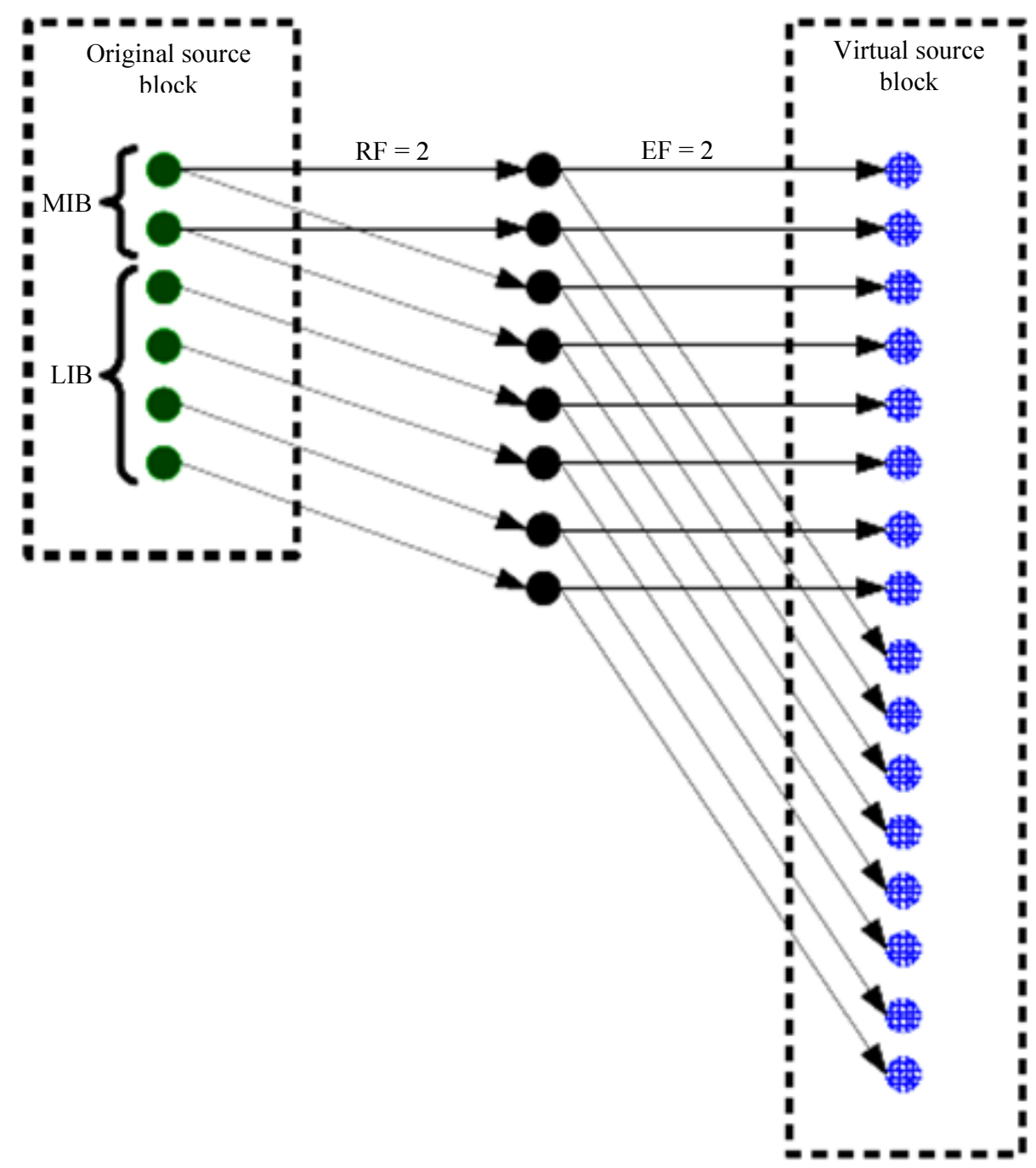

Fig. 26: Recent UEP scheme: Building a virtual source block with the UEP scheme proposed in (Ahmad et al., 2009). Here k $=6$, $\mathrm{EF}=2$ and $\mathrm{RF}=2($ Courtesy of (Ahmad et al., 2009)) 


\section{Information symbol \\ Encoded symbol}

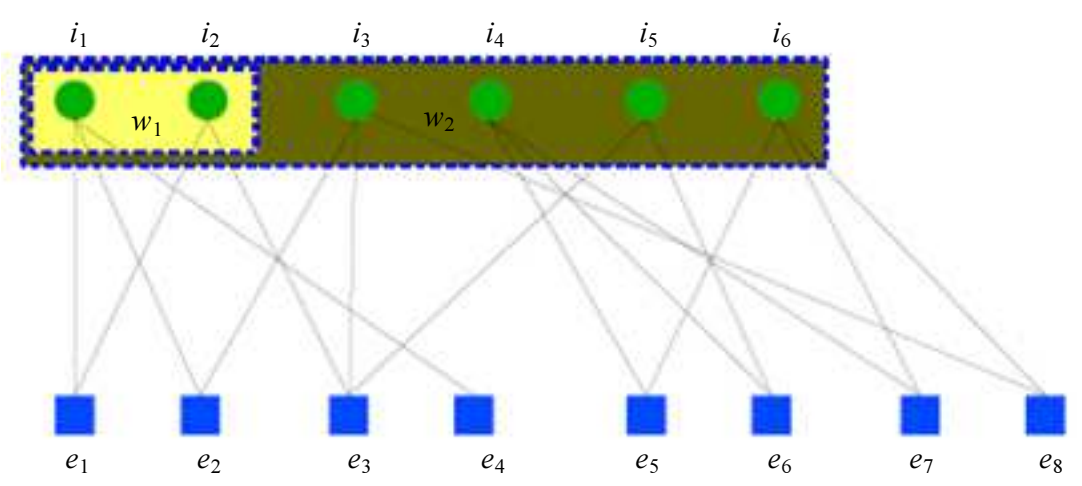

Fig. 27: UEP scheme 2 (proposed in (Sejdinovic et al., 2007)): Two levels of protection are used. The encoded symbols e1 and e4 are generated from the MIB class while the remaining encoded symbols are generated from the LIB class (Courtesy of (Ahmad et al., 2009))

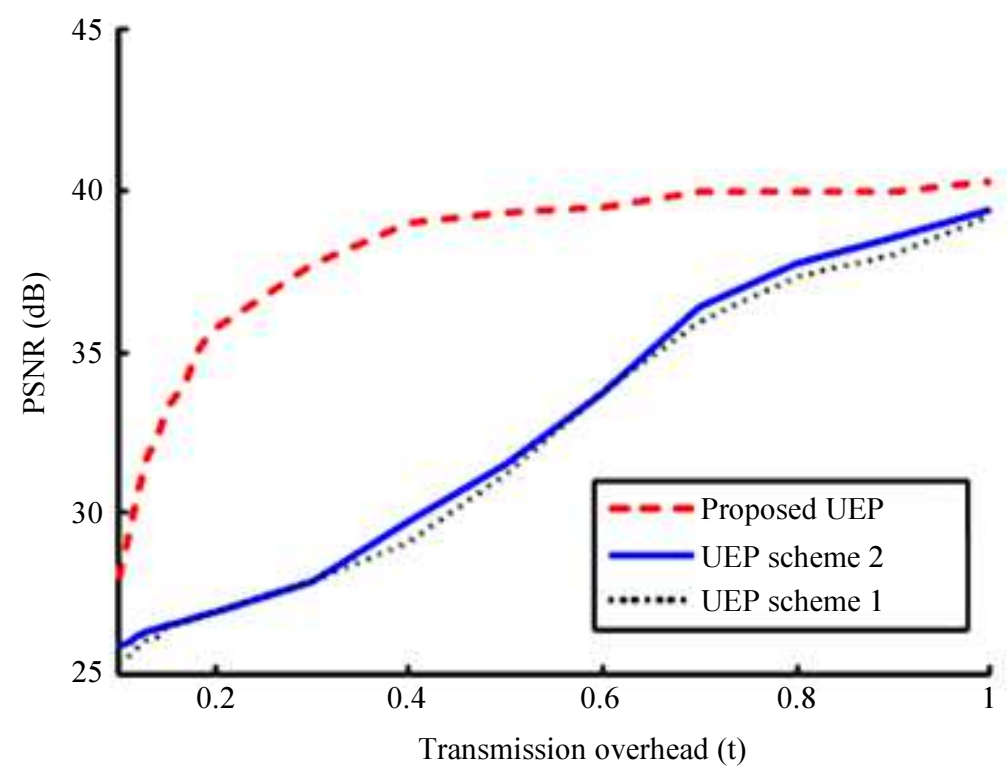

Fig. 28: PSNR of receiver class with symbol loss rate 0 as a function of the transmission overhead (Courtesy of (Ahmad et al., 2009)

UEP scheme 2 of (Sejdinovic et al., 2007) (Fig. 27) and the Recent UEP scheme of (Ahmad et al., 2009), Then they concluded the following:

1) Applying the recent static UEP technique leads to a better system performance (e.g., PSNR) than the previous EEP techniques especially in real-time video streaming over packet erasure networks (Ahmad et al., 2009; Pishro-Nik et al., 2005; Rahnavard and Fekri, 2006; Rahnavard et al., 2007a; Ahmad et al., 2008b)

2) Also the Recent static UEP scheme (with $R F=2$, $\mathrm{EF}=2$ ) has a better PSNR performance than the
Recent static UEP scheme (with $\mathrm{RF}=2, \mathrm{EF}=3$ ) and Recent UEP scheme (with $\mathrm{RF}=3, \mathrm{EF}=2$ )

3) Figure 28 shows that the recent static UEP scheme of (Ahmad et al., 2009) (with $\mathrm{RF}=2, \mathrm{EF}=2$ ) achieved better PSNR performance (by at most $13 \mathrm{~dB}$ ) than the previous static UEP scheme 1 (Rahnavard et al., 2007b) (Fig. 25) and static UEP scheme 2 (Sejdinovic et al., 2007) (Fig. 26) for unicast video transmission scenario (Ahmad et al., 2011)

Finally, the results of the simulation experiments for multicast video transmission scenario in (Ahmad et al., 2009; 2011) shows that the recent static UEP scheme 
has a better average PSNR performance when the transmission overhead is high and a worse performance when the transmission overhead is low. Therefore the Recent (Ahmad et al., 2009) static UEP scheme is more suitable for the applications where the high video quality is required.

Basically, the simulation experiments in (Ahmad et al., 2011) showed that the recent (Ahmad et al., 2009; 2008b) static UEP scheme increased the encoding and decoding time especially when they increased the EF, But in other hand the encoding and decoding time of recent static UEP scheme were still low enough and suitable for real-time video streaming applications. Also the BER for both the MIB symbols and the LIB symbols was rapidly decreased when they applied recent static UEP scheme. Furthermore, in (Ahmad et al., 2011) they showed that the best average PSNR performance results for the recent static UEP scheme were achieved when $\mathrm{RF}=2$ and $\mathrm{EF}=20$.

To conclude, the recent (Ahmad et al., 2009; 2008b; 2011) static UEP have a crucial role in increasing the quality of real time and live video streaming over packet erasure networks. To be more specific, the recent (Ahmad et al., 2009; 2009; 2008b) static UEP achieved better PSNR performance (by at most $13 \mathrm{~dB}$ ) than the previous UEP scheme 1 (Rahnavard et al., 2007b) (in Fig. 25) and UEP scheme 2 (Sejdinovic et al., 2007) (in Fig. 26) while the encoding and decoding time of recent static UEP scheme were still low enough and suitable for realtime video streaming applications.

Next sub-section highlights adaptive UEP technique and shows that adaptive UEP settings bring significant enhancements in terms of average PSNR over the static UEP techniques for same used bandwidth.

Enhancing the Unicast and Multicast Video Streaming via using the Adaptive Unequal Error Protection with Rateless Luby Transform (LT) Codes

Zanbouri et al. (2014) we proposed a heuristic based algorithm to adapt the value of EF, the number of priority classes and value of RF for each class dynamically according to the observed packet loss rate for each receiver. Figure 29 shows the block diagram of the proposed system for three multicast receivers. Each receiver observes the packet loss rate and sends it periodically to the Algorithm unit in the sender using the feedback channel. The algorithm uses packet loss rate from all receivers to calculate the value of $\mathrm{EF}$, number of classes and value of RF for each class. In the following we give our heuristic based algorithm assuming temporal scalability:

1) If the average packet loss rate is less than $2 \%$, then: a) The source symbols should be divided into three classes: MIB (I frames), Average Important Bits (AIB) (P frames) and LIB (B frames)

b) Set RF to 3 for MIB, set RF to 2 for AIB and set RF to 1 for LIB symbols

c) Set EF to 20

2) If the average packet loss rate is less between $2 \%$ and $15 \%$, then:

a) The source symbols should be divided into two classes: MIB (I and P frames) and LIB (B frames)

b) Set RF to 14 for MIB and set RF to 1 for LIB

c) Set EF to 1

3) If the average packet loss rate is greater than $15 \%$, then:

a) The source symbols should be divided into two classes: MIB (I and P frames) and LIB (B frames)

b) Set RF to 20 for MIB and set RF to 1 for LIB

c) Set EF to 1 for all MIB and LIB symbols to achieve the final virtual source block

In (Zanbouri et al., 2014) we concluded that the adaptive UEP scheme outperforms all static (Ahmad et al., 2009; 2008b; Sejdinovic et al., 2007) UEP scheme settings of RF and EF across the whole range of packet loss rates from 0 to $30 \%$. And this because that the adaptive UEP scheme since adapts its UEP settings dynamically with the current loss rate, it achieves better PSNR for the whole range of packet loss rates.

\section{The Layered Video Streaming}

The video streaming actually consisted of several layers, typically one base layer and many enhancement layers. The base layer provides an acceptable basic video quality (or provides the most important information), whereas the higher enhancement layers help in refining the video quality of lower layers. As a matter of fact that if the receiver receives more video layers then it leads to better video streaming quality. However, most of layered video streaming techniques give higher priority to more important layers, therefore the receiver can receive the base layer with a high probability (Liu et al., 2009a).

In sub-section VII.A, we demonstrate the Layered Video Multicast with Retransmissions (LVMR) technique and then sub-section VII.B presents the Layered Quality Adaptation for Internet Video Streaming by implementing some buffering techniques at the receiver end. Finally, in sub-section VII.C we talk about the H.264/SVC compression standard and how it supports layered video streaming. 


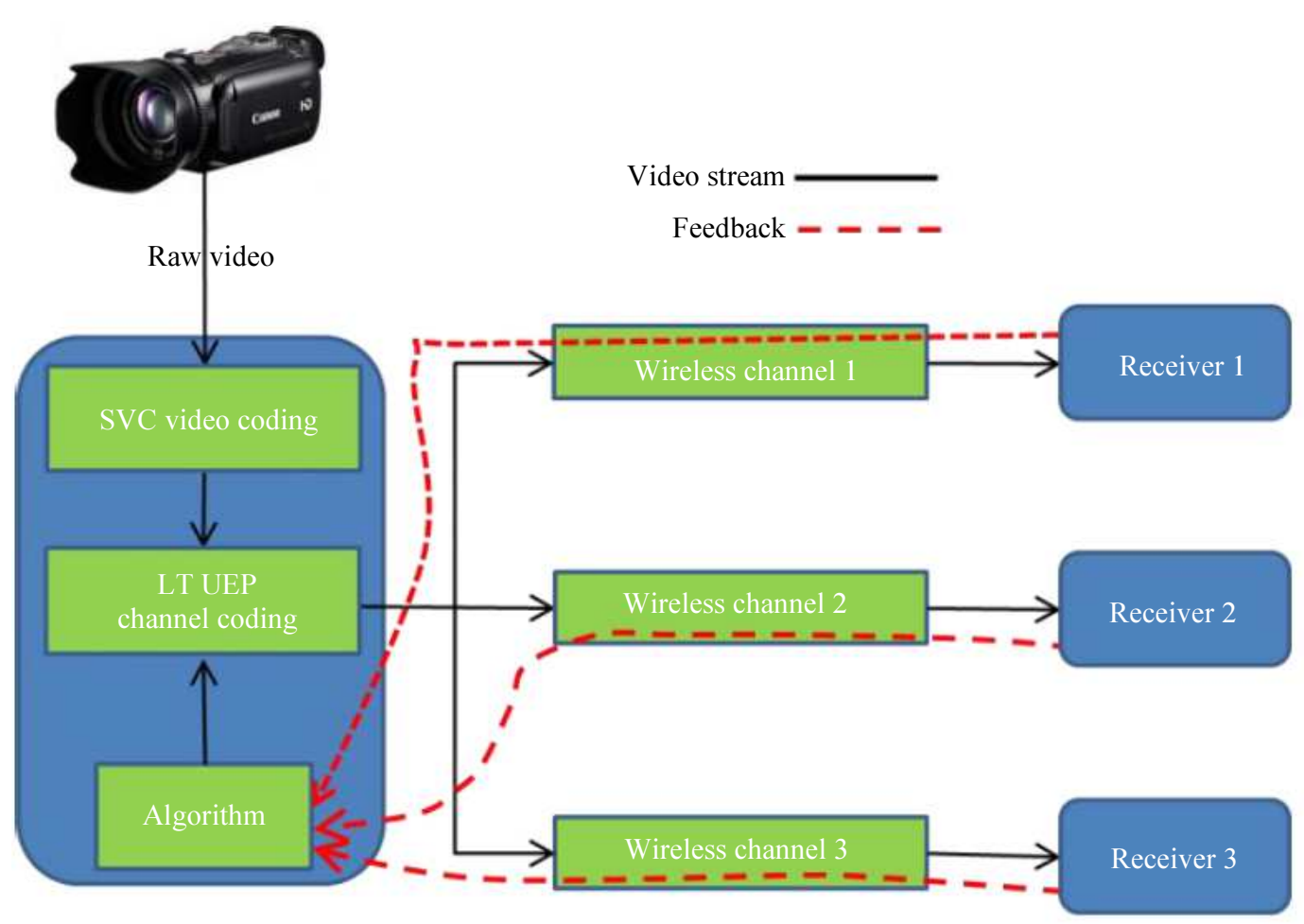

Fig. 29: The Proposed UEP scheme (Courtesy of (Zanbouri et al., 2014))

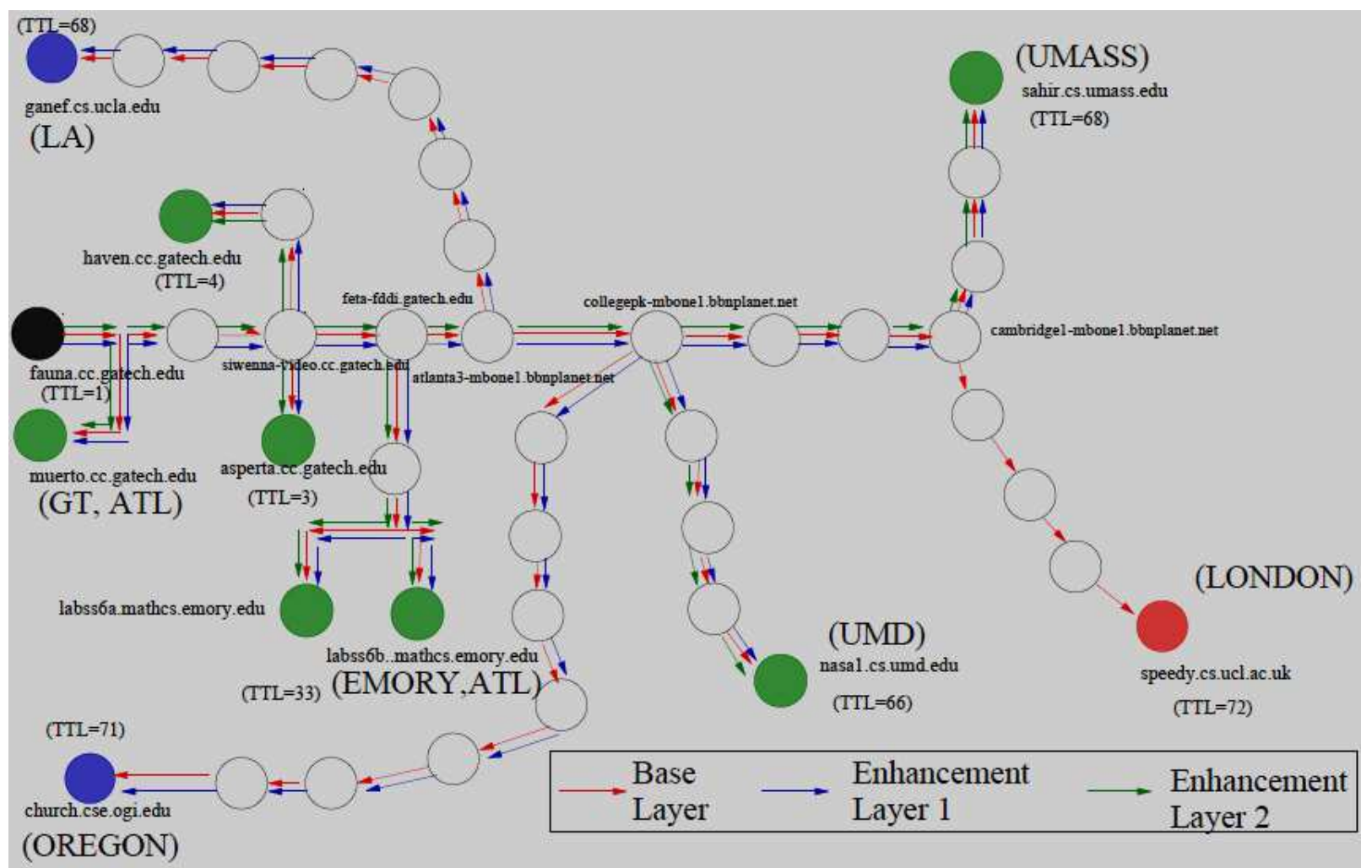

Fig. 30: An example of multicast Video Streaming Layers between the sender and many receivers (Courtesy of (Li et al., 1998)) 
Layered Video Multicast with Retransmissions (LVMR) and Hierarchical Rate Control Techniques

Layered Video Multicast with Retransmission (LVMR) is a protocol that proposed to manage the video streaming over packet erasure networks with heterogeneous multicast receivers (Li et al., 1998). In (Li et al., 1998), they supposed that the video streaming is consisted of many layers, one base layer (or basic level of video quality) and some enhancement layers (Fig. 30). Furthermore, in LVMR (Li et al., 1998), they proposed BEC technique that:

1) Enhances the quality of receiving within each layer by adding a retransmitting of lost packets technique (Li et al., 1998)

2) And adapts the network congestion and heterogeneity by adding hierarchical rate control technique to support the adding and dropping of video layers by receiver (as shown in Fig. 31) (Li et al., 1998)

On the other hand BEC, Hierarchical Rate Control and Packets Retransmission techniques were too slow and therefore not suitable for real-time and live video streaming applications.
Layered Quality Adaptation for Internet Video Streaming by Using Some Buffering Techniques at the Receiver End

We need to deal with two conflicting requirements to handle the video streaming applications over the packet erasure networks (e.g., the Internet):

1) The video streaming application requirements: According to the QoS requirements, the video streaming application is delay-sensitive, lost packettolerant and rate-based. Therefore the video steaming requires a relatively stable bandwidth to deliver a video streaming with a certain quality

2) Network requirements: Indeed, Internet is shared and unstable type of network, also there should be cooperation between the senders and the receivers to solve congestion problems. Therefore, in (Rejaie et al., 2000) they supposed that the TFRC (see sub-section IV.B.ii) was used as a congestion control mechanism, thus the network bandwidth will be changed according to the congestion state

Rejaie et al. (2000), they proposed a technique to adjust the layered quality of congestion controlled video streaming playback on the fly (Fig. 32).

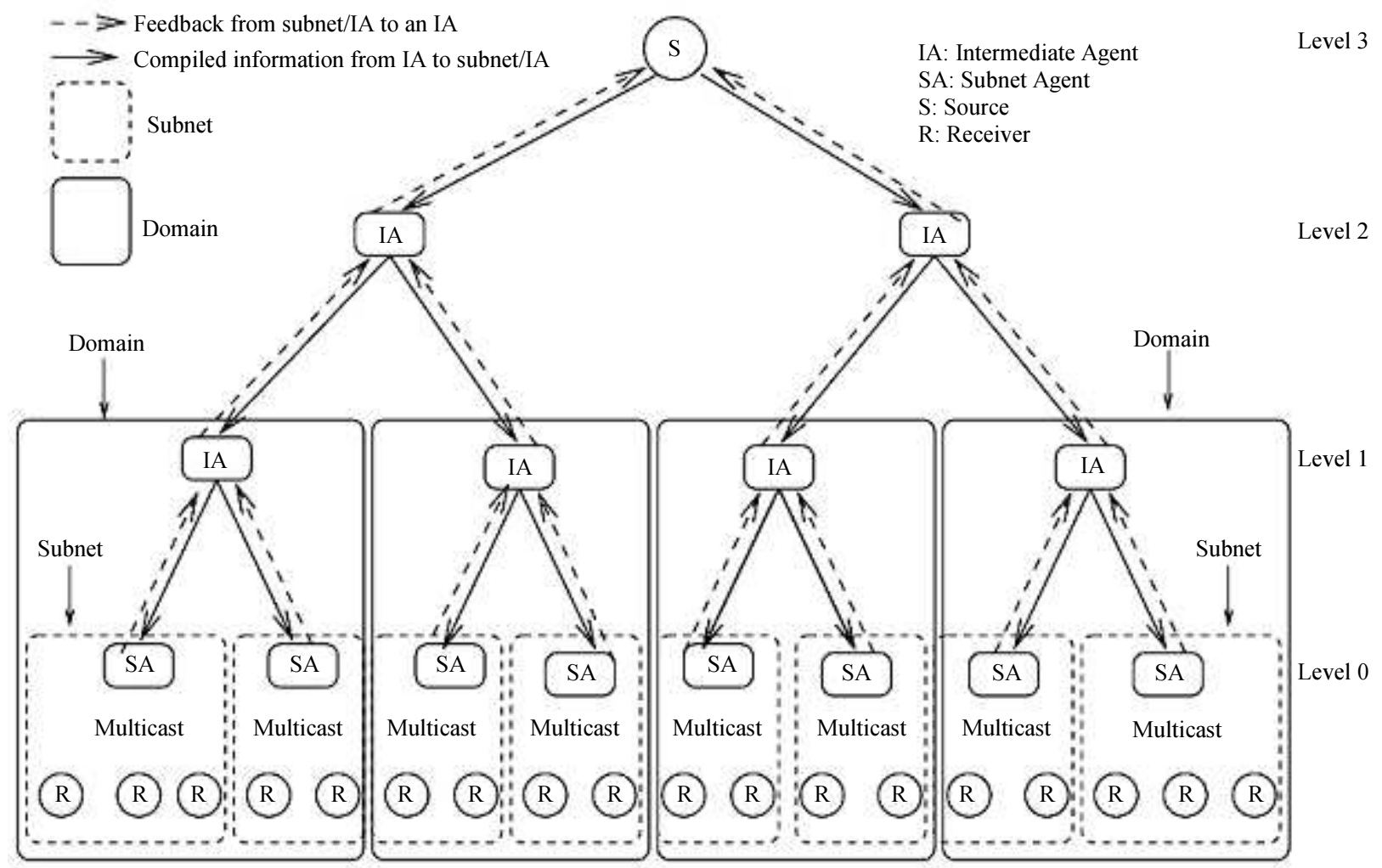

Fig. 31: Hierarchical Control Architecture used in LVMR (Courtesy of (Li et al., 1998)) 


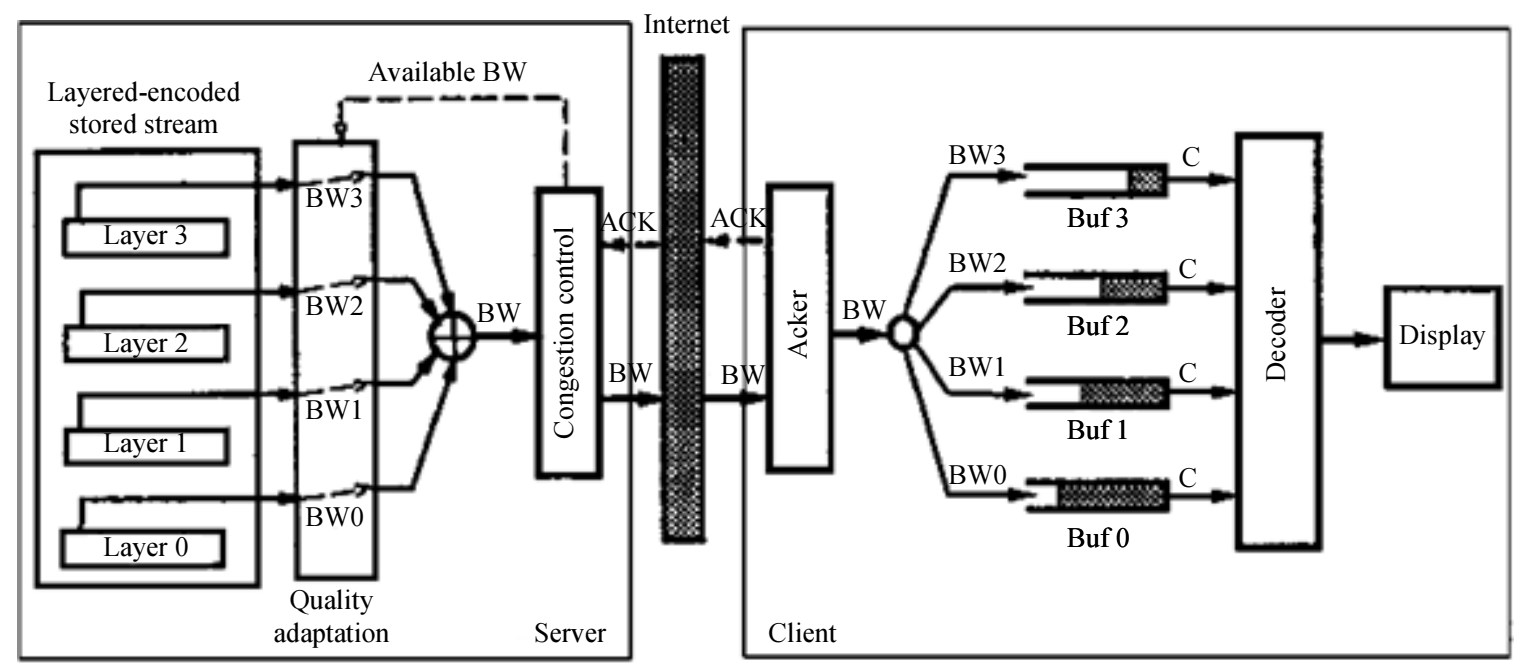

Fig. 32: End-to-end components of layered quality adaptation mechanism (Courtesy of (Rejaie et al., 2000))

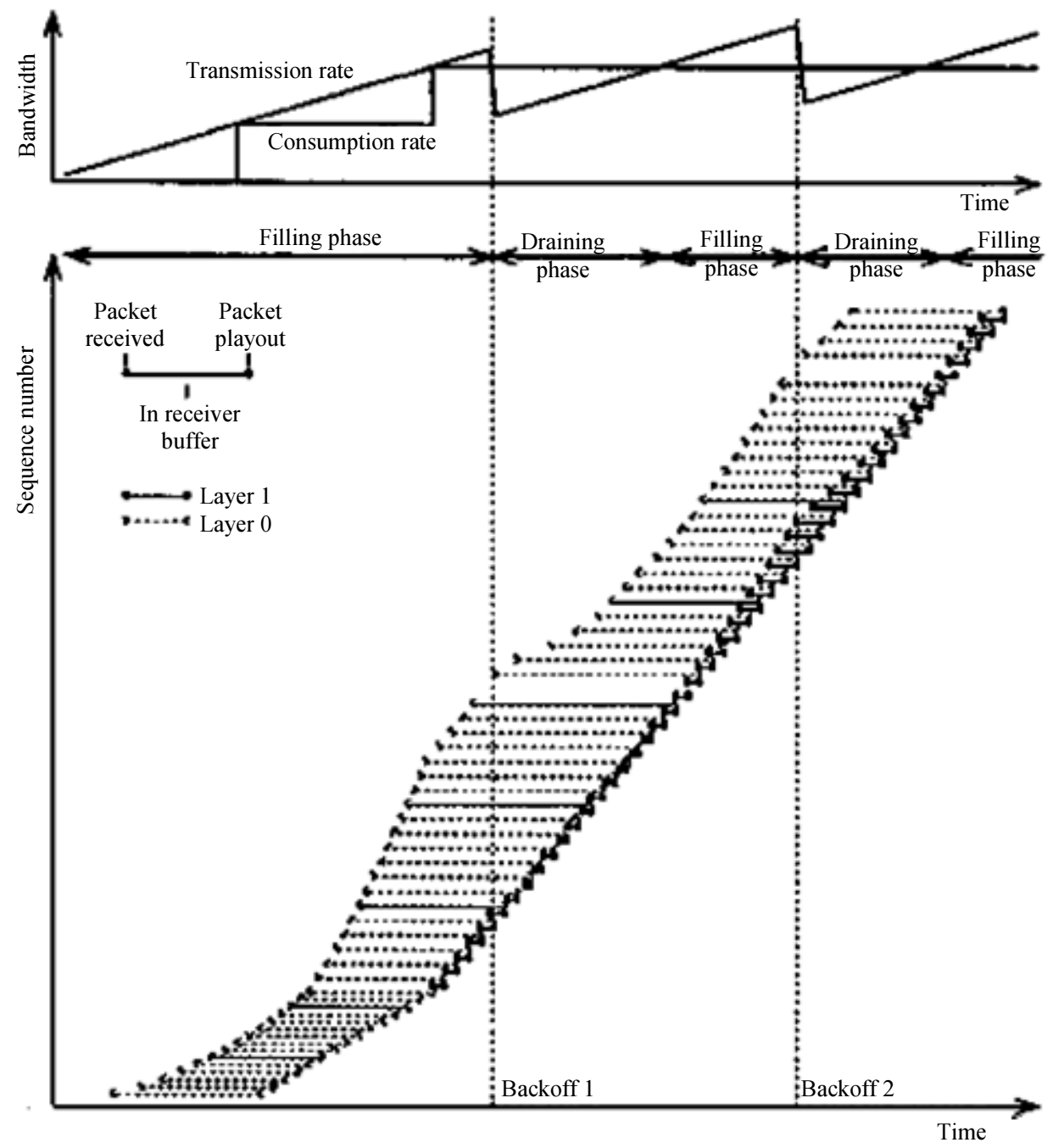

Fig. 33: The scenario of applying Layered encoding with receiver buffering technique (Courtesy of (Rejaie et al., 2000)) 


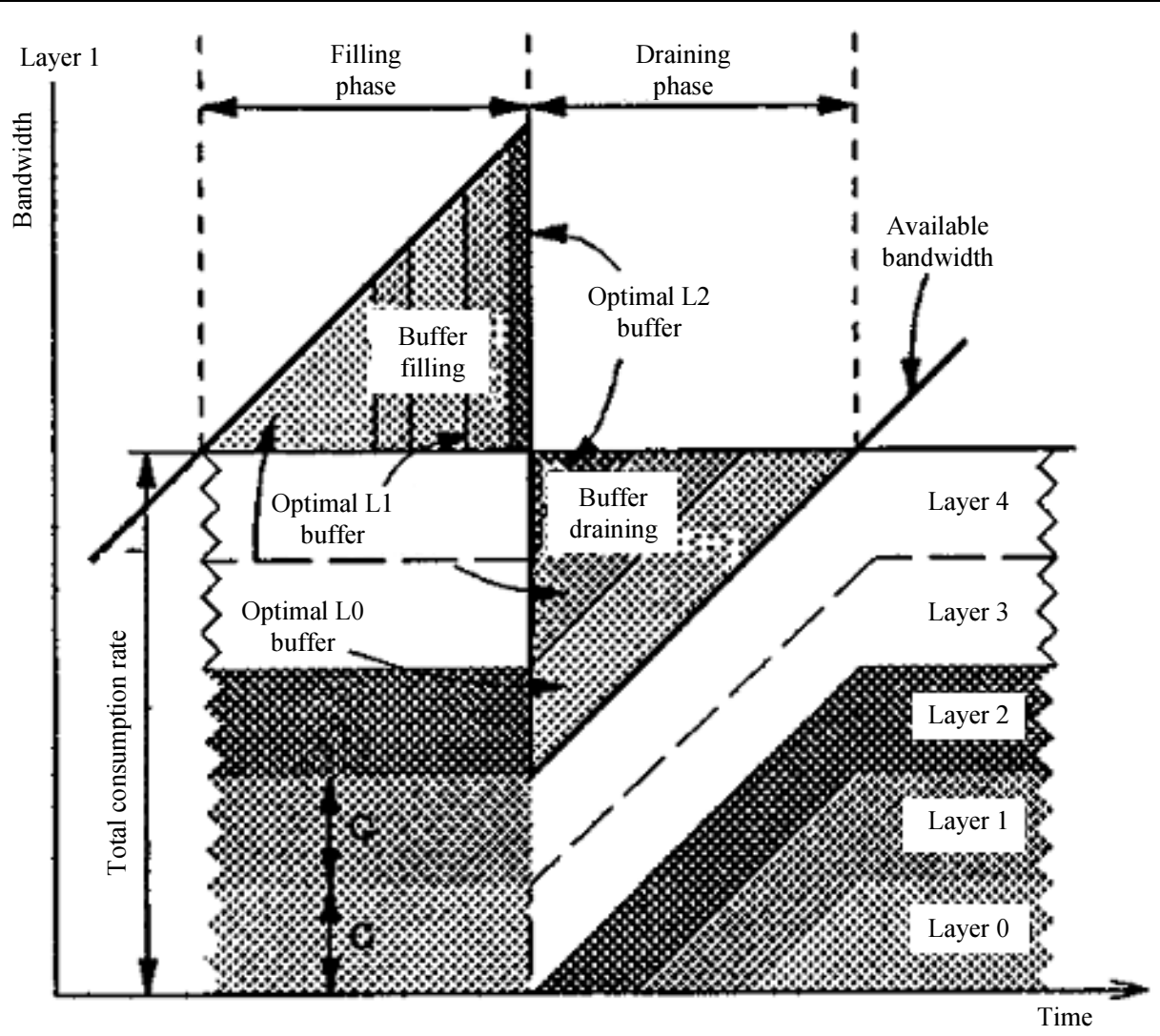

Fig. 34: Optimal buffer sharing (Courtesy of (Rejaie et al., 2000))

Furthermore, in (Rejaie et al., 2000) they supposed that there is a mechanism for adjusting the buffer distribution (Fig. 33 and 34); as the available bandwidth varies by carefully allocating the bandwidth among the active layers (Rejaie et al., 2000). Therefore and as a result of using buffering technique at the receiver side, then they solved the bandwidth mismatches between using short-term TFRC congestion control mechanism and applying the longterm hierarchical encoding and smoothing of quality. However, the buffering technique used in (Rejaie et al., 2000) was slow and as a result it is not so suitable for real-time and live video streaming applications.

\section{H.264/SVC Layered Video Coding Compression Standard}

The main difference between H.264/AVC and H.264/SVC is that H.264/AVC depends on single-layer coding technique (Liu et al., 2009a); whereas H.264/SVC depends on layered coding technique (for more details about H.264/AVC and H.264/SVC you can refer to subsection III.F and sub-section III.G). As a matter of fact that H.264/SVC provides flexibility to encode a video into many (more than three) layers (Liu et al., 2009a). For example, if the video streaming encoded into three layers (Fig. 35), then layer 0 (or base layer) should contain all I and P frames (Akabri et al., 2007) and layer 1should contain all Bs frames, finally layer 2should contain all B frames (for more details about I,P and B frames you can refer to sub-section III.A). On the other hand, when layer 2 Chunks (LCs 2) in a Groups of Pictures (GOP) arelost, then they will not affect the decoding of other frames in any layer; but when layer 0Chunks (LCs 0) in a GOP are not received, then they will affect decoding of all subsequent frames in this GOP. On the other hand, H.264/SVC coding is also suitable for UEP, since it naturally divides the content into layers with different levels of importance: A base layer which contains the most important information (or MIB information) and one or more enhancement layers with less important refinement information (or LIB information) (Liu et al., 2009a; Ffmpeg; Tourapis et al., 2005). Furthermore, in (Ahmad et al., 2009; 2008b; 2011) they showed that the Recent UEP scheme (see sub-section VI.C) achieved better PSNR performance (in H.264/SVC layered coding compression standard) than the previous UEP scheme 1 (Rahnavard et al., 2007b) and UEP scheme 2 (Sejdinovic et al., 2007), Also the encoding and decoding time of recent UEP scheme were still low enough and suitable for Broadcast/Multicast real-time video streaming applications. 


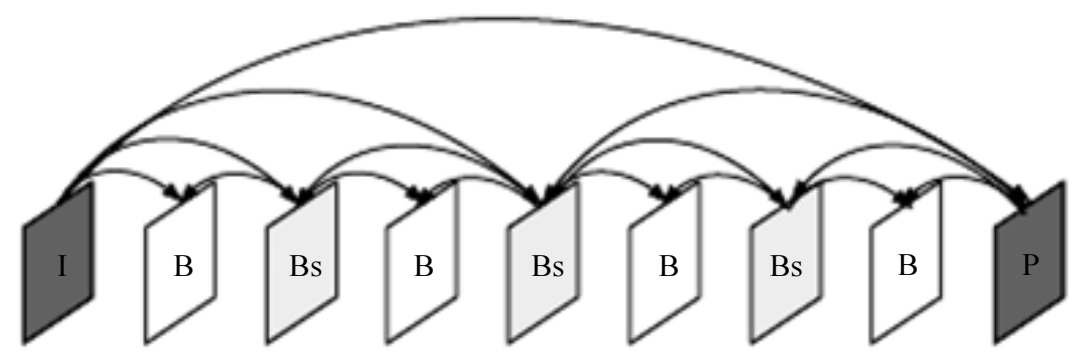

Fig. 35: One scenario for layered coding architecture of H.264/SVC (Courtesy of (Liu et al., 2009a))

Furthermore, as we mentioned in sub-section III.G that H.264/SVC is better than H.264/AVC from scalability point of view. To be more specific with H.264/SVC, a less powerful decoder can extract fewer layers and decoding fewer layers means less decoding complexity than H.264/AVC. Also, H.264/SVC has better Rate-Distortion (R-D) performance than H.264/AVC (Liu et al., 2009a; Wien et al., 2007; Schwarz et al., 2007).

To conclude, the layered coding techniques (like: H.264/SVC) can achieve better coding efficiency than single-layer coding (like: H.264/AVC). Also in (Liu et al., 2009a; Wien et al., 2007) they mentioned that the H.264/SVC encoder and decoder were more suitable for real-time and live video streaming applications than the previous layered video streaming techniques.

\section{Single and Multiple Paths Video Streaming}

In this section, we concentrate on path oriented classifications of video streaming. Therefore, in subsection VIII.A we present the single path video streaming, then in sub-section VIII.B we talk about the multiple path video streaming and its advantages compared to single path video streaming.

\section{Single Path Video Streaming}

Here they supposed that the video can be streamed over single path (or traditional method) of the existing paths between the sender and the receiver through the Internet (Ghareeb, 2008). But on the other hand, the single path video streaming leads to reducing the scalability and deployment over the Internet, also it increases the congestion over the Internet.

\section{Multiple Paths Video Streaming}

In order to solve the single path video streaming problems, they proposed several multipath video streaming techniques. The main goal of these multipath video streaming techniques is to achieve an optimal quality of service over delay-constrained video applications. Furthermore, sending packets through multiple paths will increase the capability to adapt with dynamic network conditions (Ghareeb, 2008). Finally, multipath video streaming leads to reducing the impact of packets losses.

In this tutorial paper we highlight the following three types of multiple path video streaming:

\section{Applying Multipath Video Streaming between Single Sender and Single Receiver}

In (Ribeiro et al., 2005) they supposed that there was path diversity between single sender and single receiver. Furthermore, they supposed that the number of paths can be increased up to a certain threshold value. In fact, path diversity leads to dividing the load between many paths which reduces the overall traffic (Ribeiro et al., 2005) and hence leads to reducing the packet loss probability and as a result enhancing the quality of services on delay-constrained video applications. However, the worst case scenario will be happened when all the paths share a common bottleneck segments, therefore it will increase the packet loss level and as a result have a bad effects on the quality of services. By the way, multiple paths video streaming still have less packet loss rate than single path video streaming.

\section{Applying Multipath Video Streaming from Many Senders to Single Receiver}

Apostolopoulos et al. (2002) they supposed that many senders can send video streaming to a single receiver. In this study they proposed that the performance of video streaming can be enhanced (low latency, scalability, fault tolerance, reduce the probability of loss packets) by exploiting the path diversity provided by existing Content Delivery Network (CDN) infrastructure in addition to multiple description (MD) coding representation (for more details about MD coding you can refer to sub-section V.A) of MPEG-4 and H.263. In CDN, they enhance the end-user performance by caching popular content on edge servers located closer to users and hence leads to reducing the request response time and the probability of packet loss. Dynamic CDN provides path diversity between different paths that existed between a client and its closest edge 
servers. Thus MD coding with path diversity (or MD$\mathrm{CDN}$ ) is one of the proposed technique to deal with real time video streaming and as it is mentioned in subsection V.A that the quality of the decoded signals can be improved with the greater number of the correctly received descriptions.

There was another intelligent multipath video streaming approach between multiple senders and single receiver presented in (Ghareeb, 2008; Chow et al., 2005; Abdouni et al., 2005; Franceschinis et al., 2002; Golubchik and Lui, 2002). In fact, exploring high quality video streaming was the main purpose of this approach; and this need significant bandwidth requirements without information loss (Ghareeb, 2008). In this approach they supposed that an pre-stored MPEG1 video flow can be divided into packets, then different packets take alternate routes to a single receiver. This is shown in Fig. 36 where three servers are used to stream data over a wide area network. Any server can send any part of data; specifically, server $i$ sends part $\alpha i$ of the data expected by the receiver, where $0 \leq \alpha i \leq 1$ and $\Sigma_{i} \alpha i=1$. This can be done by determining a sending pattern for each server, as shown in Fig. 36 where each sender only sends packets depicted by the solid rectangles. As each packet is sent by only one of the senders, the total amount of data sent is the same as in a single path case. Thus, the overall load on the network will not be increased using this approach.

The results as showed in (Talari and Rahnavard, 2010) indicate that multipath streaming leads to better loss characteristics than single path streaming with or without the use of an erasure code. Furthermore, (Abdouni et al., 2005) talks about the load distribution problem. It concentrated on determining an appropriate optimization objective for computing the load distribution. Then it conducts a performance study to understand the benefits of these optimization objectives.

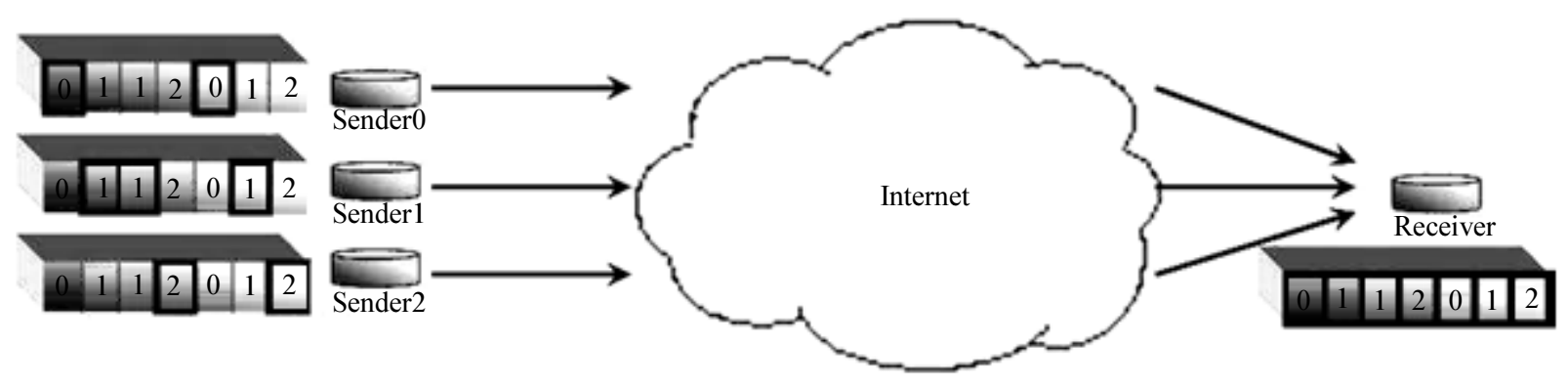

Fig. 36: Different packets from many senders to single receiver (Courtesy of (Ghareeb, 2008))

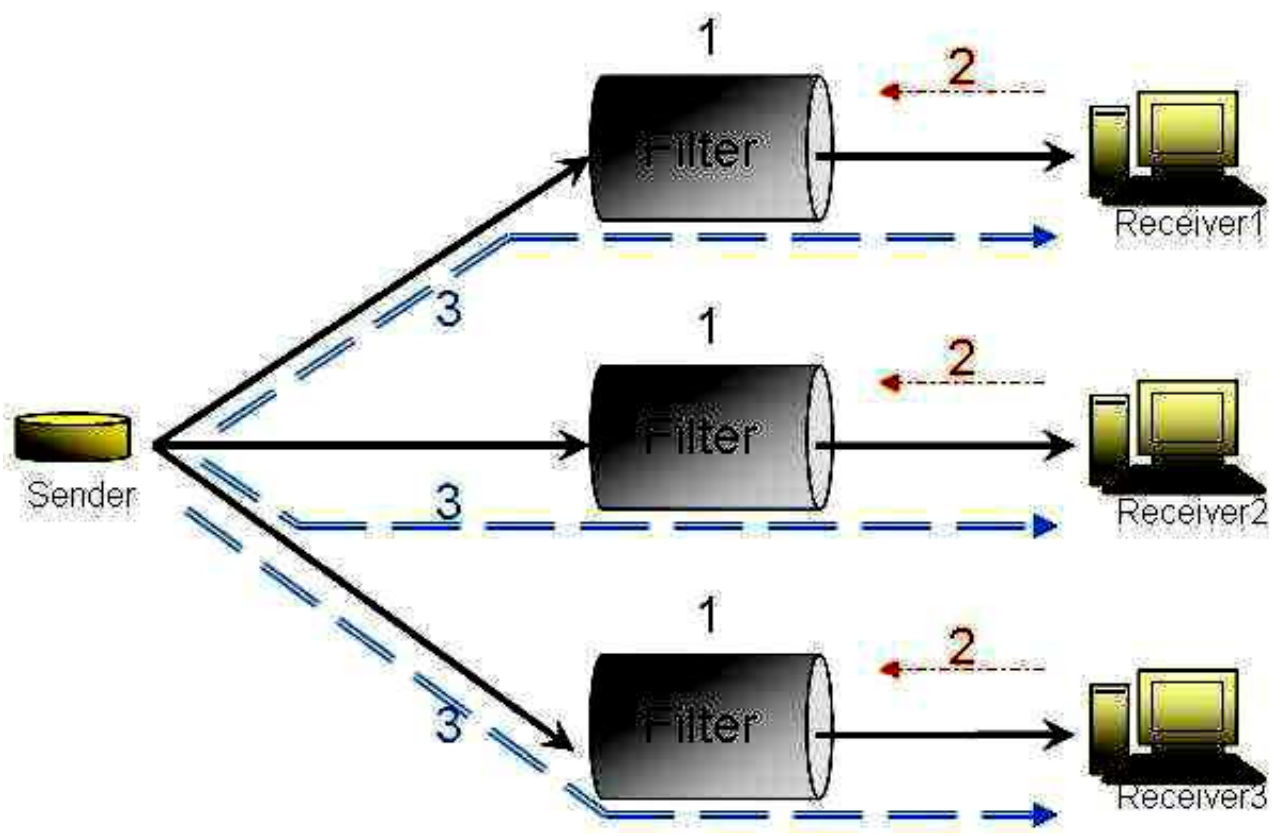

Fig. 37: The multicast tree of MHPF between single sender and many receivers (Courtesy of (Ghareeb, 2008)) 
In (Talari and Rahnavard, 2010), they applied flexible Distributed Rateless Codes with UEP scheme 1 (proposed in (Rahnavard et al., 2007b)) technique (or we can call it DU-rateless codes) to the multiple senders and single receiver method. In this technique they supposed that we can assign various data block lengths $(\mathrm{n})$ to different senders according to their importance level in construction the video streming at the receiver end. To be more clear, in (Talari and Rahnavard, 2010) they supposed that the more important data need larger data block length (n) than the less important data. Moreover, the simulation results in (Talari and Rahnavard, 2010) showed that DU-rateless codes technique leads to better PSNR with minimal error rates.

However, there were some disadvantages of using multipath video streaming (from many senders to single receiver) approaches, starting with the potential costs of using multiple senders (Lun et al., 2006), then the overheads related to sending data over multiple paths and then assembling it into a single stream at the receiver should also be considered. Finally, these approaches supposed that the multiple paths have always disjoint bottleneck nodes, which is not always true over real Internet.

\section{Applying Multipath Video Streaming from Single Sender to Multiple Receivers}

Here in this subsection we supposed that single sender can stream many copies of the same video to many receivers, or we can call it multicast video streaming (Lun et al., 2006). The multicast video streaming solution came to deal with the interactive and teleconferencing video applications over Internet. In (Lee et al., 2000) they presented an overlay network architecture called Multicast Heterogeneous Packet Flows (MHPF) that supports multicast video streaming of heterogeneous data for small to medium multicast groups without decomposing them into component homogeneous data streams (Fig. 37). In (Lee et al., 2000), the MHPF server can multicast the video to many receivers over multicast tunnels. The MHPF servers implement a specialized packet forwarding technique (rate adaptation and priority-based filtering on each multicast tunnel), therefore only the highest priority packets can be sent over the downstream paths. In the other hand, each receiver can periodically generate feedback (about the bandwidth on the path leading to itself) over the upstream path. When the network circumstances is dynamically changing, MHPF effectively responses to these circumstances.

But on the other hand, the performance of MHPF will be degraded when the number of receivers increases; also we should to take into consideration the cost of multicast same video streaming to many receivers (Lun et al., 2006).

\section{Video Streaming Over Wireless Networks}

As a matter of fact that the wireless networks (e.g., 3G Mobile phone system, Bluetooth, Wi-Fi and WiMAX) are the most unstable types of packet erasure networks, also these networks have the following disadvantages with comparison to the wired networks (Rodriguez, 2011; Caro, 2004):

1) The wireless networks always achieve less data rate bandwidth than the wired networks

2) Also the wireless networks suffer from unpredictable bandwidth issues

3) The wireless networks suffer from reliability, fading and interference issues

4) Finally, the wireless networks suffer from dynamic fluctuation in the congestion level, channels errors, lost packets rate and PSNR

Therefore there are a lot of technical issues arise when the wireless networks trying to meet the requirements of high throughput, low delay and acceptable lost packets rate for live and real-time video streaming transmission. In sub-section IX.A, we show a brief overview about $3 \mathrm{G}$ mobile phone system, then in sub-section IX.B we present Wi-Fi Ad-hoc networks, in sub-section IX.C we show WiMAX networks, after that in sub-section IX.D we make a small comparison between Wi-Fi and WiMAX networks. Then in sub-section IX.E we present how to use a hybrid of Wi-Fi (802.11 n) and WiMAX (802.16 $\mathrm{m})$ networks. Finally, in sub-section IX.F we show some examples of enhancing the video streaming quality over the wireless networks.

\section{$3 G$ Mobile Phone System}

$3 \mathrm{G}$ is the third generation of mobile phone technology or we can call it Universal Mobile Telecommunications System (UMTS), also it has the ability to handle voice and all types of data effectively, including video and audio in the mobile domain, so it can deal with voice and data seamlessly, or we can say that $3 \mathrm{G}$ mobile phone system is data centric. Moreover, 3G relies on Wideband Code Division Multiple Access (WCDMA) radio technology (3GPP, 2005). 3Gprovides high-speed data rate transfer, for example it can achieve 384 kilobits per second (kbits/s) for moving vehicles and minimum 2 megabits per second (Mbits/s) for stationary users or pedestrians (ITU, 2008).

\section{The Wi-Fi Ad-Hoc Networks}

Here in this tutorial paper we relied on $802.11 \mathrm{n}$ Wi-Fi Ad-hoc network instead of other Wi-Fi Ad-hoc 
networks for the following reasons (Phifer, 2010; Chou et al., 2009):

1) $802.11 \mathrm{n}$ has a maximum indoor range which is about 70 meters (as shown in Table 1)

2) Also $802.11 \mathrm{n}$ has a maximum outdoor range which is about 250 meters (as shown in Table 1)

3) Finally $802.11 \mathrm{n}$ has a maximum bandwidth which is $40 \mathrm{MHz}$.

\section{The WiMAX Networks}

In this tutorial paper we relied on $802.16 \mathrm{~m}$ WiMAX network instead of other WiMAX networks for the following reasons (Dhawan, 2007):

1) $802.16 \mathrm{~m}$ has a maximum range of about 30 -mile $(50-\mathrm{km})$ radius from base station

2) $802.16 \mathrm{~m}$ has an advanced Air Interface with data rates of $100 \mathrm{Mbit} / \mathrm{s}$ mobile and $1 \mathrm{Gbit} / \mathrm{s}$ fixed

\section{Wi-Fi Vs. WiMAX Networks}

From the Table 2 and (Chou et al., 2009; Dhawan, 2007; Ibáñez et al., 2008) we can conclude the following:

1) The cost: Wi-Fi network (802.11 n) is free, But WiMAX (802.16m) need a license from the government (Dhawan, 2007)]

2) The coverage range: The coverage range of WiMAX $(802.16 \mathrm{~m})$ is around $50 \mathrm{~km}$, but the coverage range of Wi-Fi network $(802.11 \mathrm{n})$ is around $100 \mathrm{~m}$

3) The Frequency range: The frequency range (2.4 $\mathrm{GHz})$ in Wi-Fi network (802.11 n) is less than the frequency range (up to $11 \mathrm{GHz}$ ) in WiMAX $(802.16 \mathrm{~m})$

4) Network topology: WiMAX $(802.16 \mathrm{~m})$ is an infrastructure network, whereas Wi-Fi network (802.11 n) can be used in both infrastructure and Ad-hoc networks. Therefore Wi-Fi network
(802.11 n) is more flexible than WiMAX (802.16 m) (Dhawan, 2007)

5) The performance evaluation in less than $100 \mathrm{~m}$ coverage area - Especially in Indoor range: WiFi network (802.11 n) has shorter latency, higher throughput, less packets loss and better data transfer rate than WiMAX $(802.16 \mathrm{~m})$ (Chou et al., 2009; Dhawan, 2007; Ibáñez et al., 2008)

6) The performance evaluation in more than $100 \mathrm{~m}$ coverage area - Specially in Outdoor range: WiMAX network $(802.16 \mathrm{~m})$ has shorter latency, higher throughput, less packets loss and better data transfer rate than Wi-Fi (802.11 n) (Chou et al., 2009; Dhawan, 2007; Ibáñez et al., 2008)

\section{Using a Hybrid of Wi-Fi (802.11 n) and WiMAX $(802.16 \mathrm{~m})$ Networks}

In (Ibáñez et al., 2008) they proposed a good hybrid solution that uses the advantages of Wi-Fi (802.11 n) and WiMAX (802.16 m) in WLAN-WMAN (or Wi-FiWiMAX) network (As shown in Fig. 38). Also in (Ibáñez et al., 2008) they supposed that we can connect many Wi-Fi networks with single WiMAX network (As shown in Fig. 38 and 39).

In fact, the integration between WLAN and WMAN leads to more benefits for both users and service providers. Specifically, users will gain advantages such as wide network coverage and high data transmission rates; whereas service providers will achieve more economical gains because this technology leads to increasing the number of customers (Ibáñez et al., 2008).

\section{Some Examples of Enhancing the Video Streaming Quality over Wireless Networks}

In this sub section we want to present some examples of techniques for enhancing the video streaming quality over $3 \mathrm{G}$ (in sub-section IX.F.i), WLAN (in sub-section IX.F.ii) and WiMAX (in subsection IX.F.iii) wireless networks.

Table 1: Comparison between 802.11 Wi-Fi network standards (courtesy of (Phifer, 2010)) 802.11 network standards

\begin{tabular}{|c|c|c|c|c|c|c|c|c|c|c|}
\hline $\begin{array}{l}802.11 \\
\text { protocol }\end{array}$ & Release & $\begin{array}{l}\text { Freq. } \\
\text { (GHz) }\end{array}$ & $\begin{array}{l}\mathrm{BW} \\
(\mathrm{MHz})\end{array}$ & Data Rate Per stream Mbps & $\begin{array}{l}\text { Allowable } \\
\text { MIMO } \\
\text { streams }\end{array}$ & Modulation & $\begin{array}{l}\text { Appr } \\
\text { indoc } \\
(\mathrm{m})\end{array}$ & $\begin{array}{l}\text { imate } \\
\text { range } \\
(\mathrm{ft})\end{array}$ & $\begin{array}{l}\text { Appr } \\
\text { outdo } \\
(\mathrm{m})\end{array}$ & $\begin{array}{l}\text { oximate } \\
\text { or range } \\
\text { (ft) }\end{array}$ \\
\hline- & Jun 1997 & 2.4 & 20 & 1,2 & 1 & DSSS, FHSS & 20 & 66 & 100 & 330 \\
\hline a & Sep 1999 & $\begin{array}{l}5 \\
3.7^{[\mathrm{A}]}\end{array}$ & 20 & $6,9,12,18,24,36,48,54$ & 1 & OFDM & $\begin{array}{l}35 \\
-\end{array}$ & $\begin{array}{l}115 \\
-\end{array}$ & $\begin{array}{l}120 \\
5000\end{array}$ & $16000^{[\mathrm{A}]}$ \\
\hline b & Sep 1999 & 2.4 & 20 & $5.5,11$ & 1 & DSSS & 38 & 125 & 140 & 460 \\
\hline g & Jun 2003 & 2.4 & 20 & $6,9,12,18,24,36,48,54$ & 1 & OFDM, DSSS & 38 & 125 & 140 & 460 \\
\hline $\mathrm{n}$ & Oct 2009 & $2.4 / 5$ & $\begin{array}{l}20 \\
40\end{array}$ & $\begin{array}{l}7.2,14.4,21.7,28.9,43.3,57.8,65,72.2 \\
15,30,45,60,90,120,135,150^{[\mathrm{B}]}\end{array}$ & 4 & OFDM & $\begin{array}{l}70 \\
70\end{array}$ & $\begin{array}{l}230 \\
230\end{array}$ & $\begin{array}{l}250 \\
250\end{array}$ & $\begin{array}{l}820 \\
820\end{array}$ \\
\hline
\end{tabular}

A1 A2 IEEE 802.11y-2008 extended operation of 802.11a to the licensed $3.7 \mathrm{GHz}$ band. Increased power limits allow a range up to $5000 \mathrm{~m}$. As of 2009 , it is only being licensed in the United States by the FCC.

B1 B2 Assumes Short Guard Interval (SGI) enabled, otherwise reduce each data rate by $10 \%$. 
Yahia Hasan and Ali Zanbouri / Journal of Computer Science 2018, 14 (4): 499.545 DOI: $10.3844 /$ jessp.2018.499.545

Table 2: Comparison between different wireless technologies (courtesy of (Dhawan, 2007))

\begin{tabular}{llll}
\hline Technology & Bluetooth & WiFi-802.11n & WiMAX \\
\hline Application & Personal area network & Wireless LAN, internet & Broadband internet connectivity \\
Typical range & $5-20 \mathrm{~m}$ & $100 \mathrm{~m}$ & $50 \mathrm{Km}$ \\
Frequency range & $2.4-5 \mathrm{GHz}$ & $2.4 \mathrm{GHz}$ & $2-11 \mathrm{GHz}$ \\
Data rate & $2-6 \mathrm{Mbps}$ & $54-108 \mathrm{Mbps}$ & $75 \mathrm{Mbps}$ \\
Modulation & TDM & DSSS & QAM \\
Network & IP and P2P & IP and P2P & IP \\
IT network connectivity & No & YES & NO \\
Network topology & Infrastructure & Infrastructure (ad-hoc also possible) & Infrastructure \\
Access protocol & L2CAP/LMP & CSMA/CA & Request/Grant \\
Key attributes & Less cost & Wider BW, Flexibility & Throughput, coverage \\
\hline
\end{tabular}

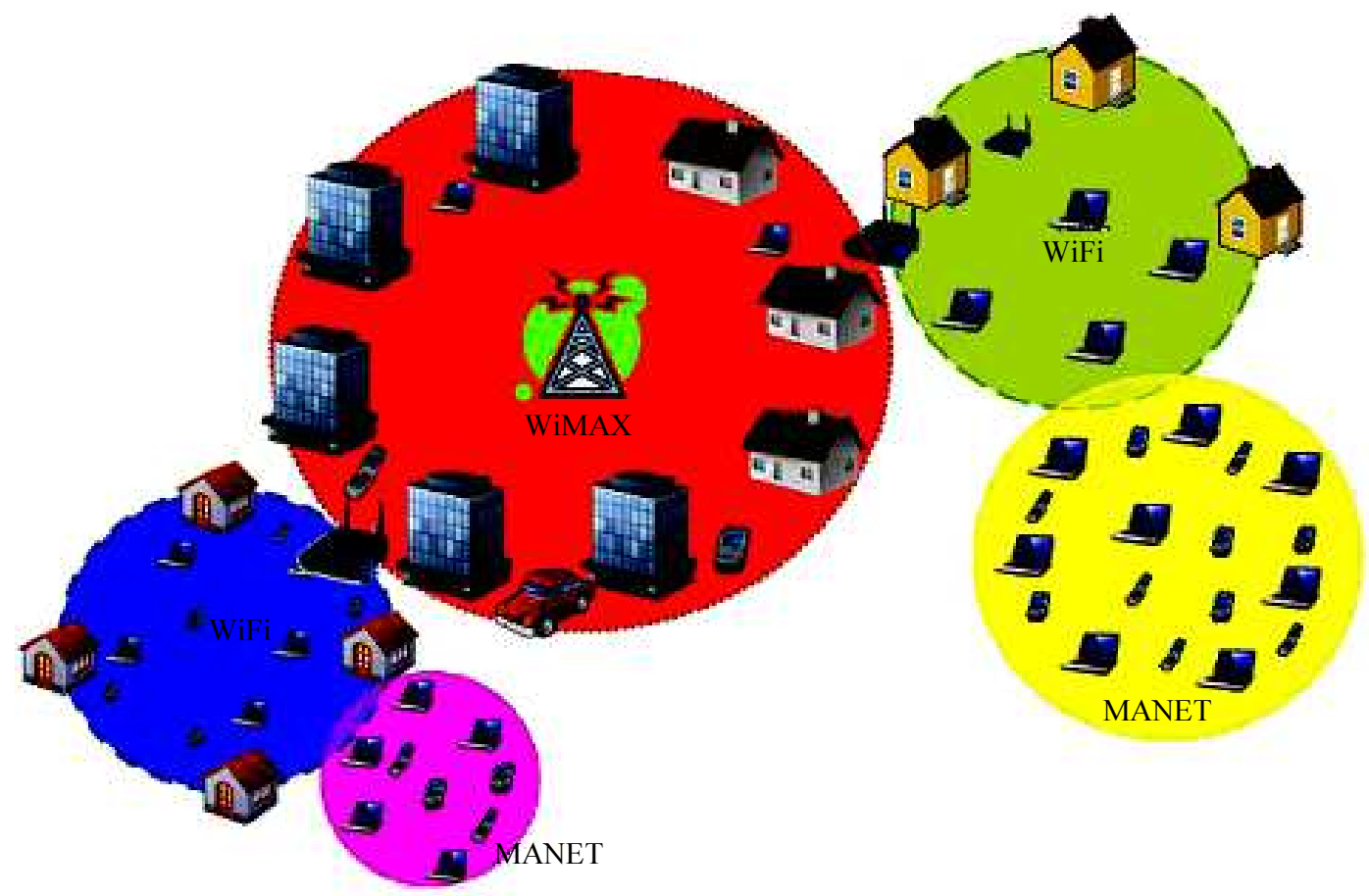

Fig. 38: The convergence between Wi-Fi and Fixed WiMAX networks (Courtesy of (Ibáñez et al., 2008))

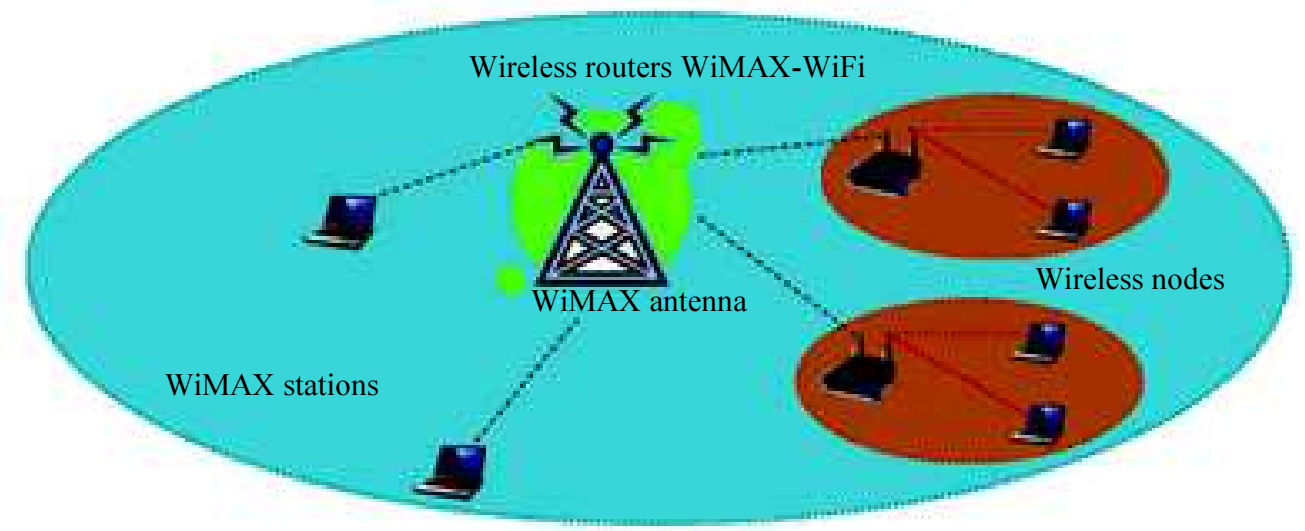

Fig. 39: Service connection from multiple Wi-Fi networks to single WiMAX network (Courtesy of (Ibáñez et al., 2008)) 


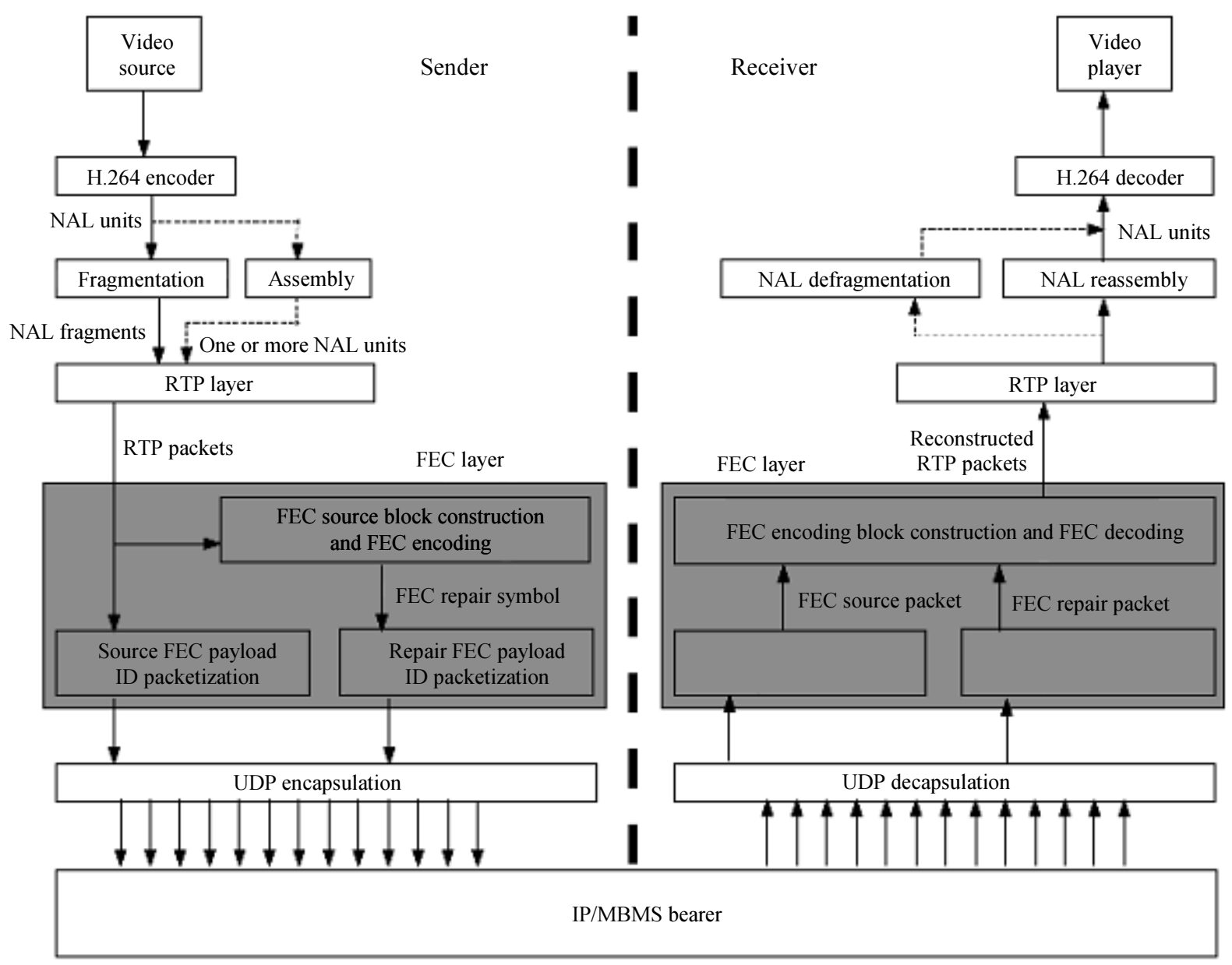

Fig. 40: MBMS video streaming transmission and receiving mechanisms (Courtesy of (Hamzaoui et al., 2008))

Some Important Techniques for Enhancing the Video Streaming Quality over $3 G$ Mobile Wireless Network

As a matter of fact that applying the real-time and live video streaming over $3 \mathrm{G}$ mobile wireless networks with limited bandwidth is really a big challenge. Actually, 3G wireless communications over erasure packet-switched networks suffers from some fading and destructive interference issues and then these issues lead to increasing the packets loss rate and decreasing the PSNR. In this sub-section we want to present some solutions for these issues.

The third Generation Partnership Project (3GPP) (3GPP, 2005) introduced Multimedia Broadcast/Multicast Service (MBMS) method (Fig. 40) for efficiently transmit the video streaming over WCDMA 3G network (Hamzaoui et al., 2008; TSGSSA, 2008; Afzal et al., 2006). In MBMS, they employed systematic Raptor FEC code at the application layer to deal with lost packets issues, also Raptor code can be applied to both the MBMS file delivery and MBMS streaming applications.
Furthermore in MBMS, they used single layer H.264/AVC video streaming compression technique; also the primary unit generated by H.264/AVC is called the Network Abstract Layer (NAL) unit. Moreover, they used RTP/UDP at transport layer to deal with real-time video streaming applications (Hamzaoui et al., 2008; TSGSSA, 2008; Afzal et al., 2006).

However, as we found in (Liu et al., 2009a; Wien et al., 2007) that the complexity of encoding and decoding processes on single layer H.264/AVC video streaming compression standard was little bit high, therefore H.264/AVC which applied on MBMS was not so suitable for real-time and live video streaming applications over $3 \mathrm{G}$ wireless networks. Then we strongly believe on that using layered H.264/SVC video streaming compression technique (refer to sub-section III.G) will lead to further enhancing the MBMS method and make it more suitable for real- time and live video streaming applications over $3 \mathrm{G}$ wireless networks.

Liu et al. (2009b), they used the H.264/SVC video streaming compression technique to improve the MBMS and due to its scalability, low decoding complexity and 
good R-D performance; so the proposed method in (Liu et al., 2009b) is suitable for real-time and live video streaming applications over $3 \mathrm{G}$ wireless networks. Furthermore, the proposed method in (Liu et al., 2009b) with H.264/SVC employed an error resilient scheme to achieve better video service quality and PSNR than the previous MBMS method with H.264/AVC. Finally 264/SVC can provide some extra functionalities like: graceful degradation in lossy transmission environments as well as bit rate, format and power adaptation.

Some Techniques for Enhancing the Video Streaming Quality over the Wireless Local Area Networks (WLANs)

Zhu and Girod (2007), they made a simple comparison between the traditional TCP and the UDPLike TCP (as shown in Fig. 41) also they showed their uses in enhancing the Video Streaming Quality over the WLANs, In UDP-Like TCP they supposed that the receiver can send just one acknowledgement (ACK) every 10 received packets not after each received packet (like in the traditional TCP) (Zhu and Girod, 2007). Then this leads to reducing the ACKs frequency in UDPLike TCP, therefore the ACKs overhead will be reduced.

Figure 41 shows that UDP-Like TCP has less packet delivery delay than the traditional TCP. To be more specific, the packet delivery delay in UDP-Like TCP did not reach to $500 \mathrm{~ms}$ in the worst cases although it in the traditional TCP reached to one second in some cases. Therefore, UDP-Like TCP will be more suitable for video streaming over the WLANs. Moreover, in (Zhu and Girod, 2007) they used H.264/AVC single-layer coding technique for compressing the video streaming.

However, from sub-section III.F, sub-section IV.B and sub-section VII.C we find that the BEC, Packets Retransmission and single-layer H.264/AVC techniques are slow and therefore not suitable for real-time and live video streaming applications over the WLANs.

On the other hand, in (Gallucci et al., 2009) they used Content-Adaptive Robust layered H.264/SVC Video streaming compression (for more details refer to subsection III.G and sub-section VII.C) technique over 802.11e Wi-Fi Ad-hoc WLANs, in this technique they applied UEP to deal with the video layers according to their importance in constructing the final video streaming and as we know that the base layer contains the most important information and the rest enhancement layers contain less important refinement information. Therefore, high priority is used for the base layer to allow the video reconstruction, even in the worst case, of a limited quality version of the content, while low priority is assigned to enhancement layers for an efficient use of network resources (Gallucci et al., 2009). Moreover, applying the H.264/SVC video streaming compression technique leads to reducing the packets loss rate and as a result leads to further enhancing the PSNR and visual quality over 802.11e Wi-Fi Ad-hoc WLANs. Finally due to the scalability, low decoding complexity (especially in Scalable Baseline profile and low level of the Scalable High profile) and good R-D performance of H.264/SVC; therefore the recent technique with H.264/SVC is more suitable for real time video streaming transmissions than the previous technique with H.264/AVC video streaming compression standard (Liu et al., 2009a; Wien et al., 2007; Gallucci et al., 2009).

Inter-Frame Retransmission to Enhance the Video Streaming Quality over WiMAX

\section{The Inter-frame Retransmission (IR) Protocol}

The proposed protocol in (Al-Akaidi and Hamzaoui, 2011) is a BEC technique. On the other hand, this protocol is proposed to find a solution for packet loss problem rather than prevent it through congestion control technique. Furthermore, in the proposed protocol (Al-Akaidi and Hamzaoui, 2011): The receiver sends one negative acknowledgement (NACK) packet to inform the sender about all lost packets within one video frame (as shown in Fig. 42).

\section{Performance Evaluation}

Al-Akaidi and Hamzaoui (2011), they used NS2 simulator to compare IR with other protocols over WiMAX network. Then after the performance comparisons between IR and (TCP, UDP, DCCP, RBUDP and BVS), they found the following (Al-Akaidi and Hamzaoui, 2011):

1) IR achieved the smallest average (packets and frames) delay by about 0.002 second (as shown in Fig. 43)

2) In comparison with UDP, DCCP and BVS; IR had a significant decrease in the packet loss rate. To be more specific, the packet loss rate is $3.5 \%$ less than UDP, $1.37 \%$ less than DCCP and $0.56 \%$ less than BVS (as shown in Fig. 44). Therefore IR achieved better throughput than those protocols.

3) However, IR had more packet loss rate and lower PSNR than TCP and RBUDP. But its short delay is still desirable for real-time video streaming.

\section{Comparison between Inter-frame Retransmission (IR) and Quick Response (QR) Techniques}

In QR the receiver sends NACK packet for every lost packet, actually this method is good for quick response in case that there is only one lost packet in the frame. On the other hand, the receiver may sends more than one NACK packet for one frame, which require more bandwidth also the sender may be frequently interrupted; Therefore it may leads to some extra delay (as shown in Fig. 45 and Fig. 46). 

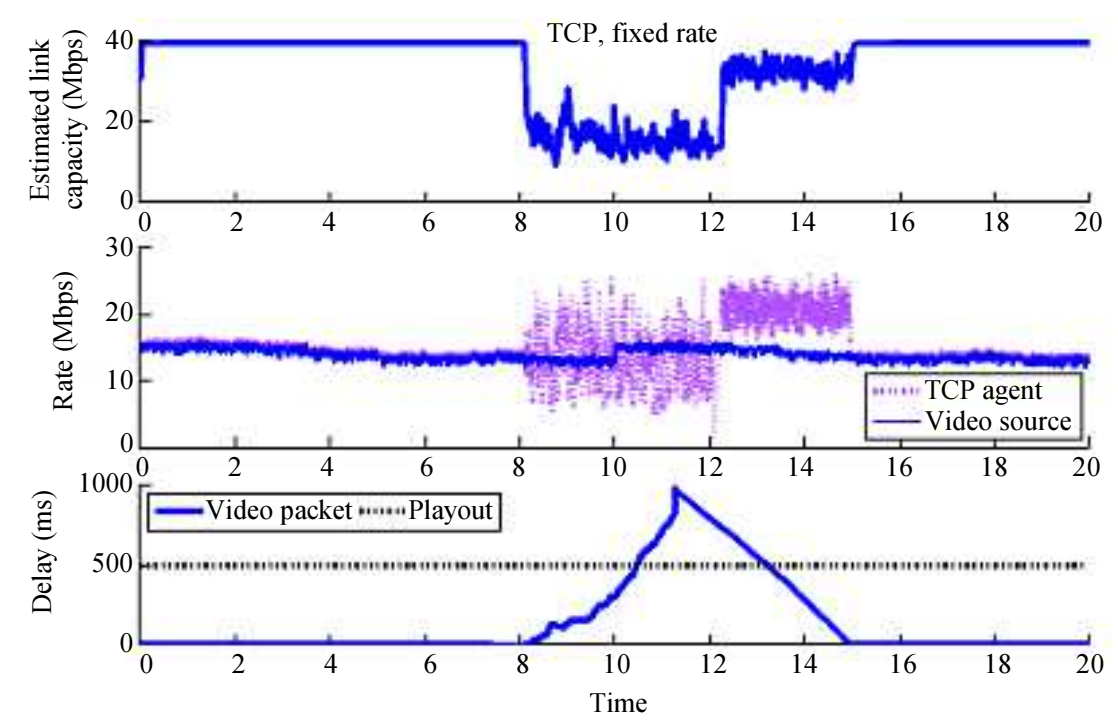

(a)
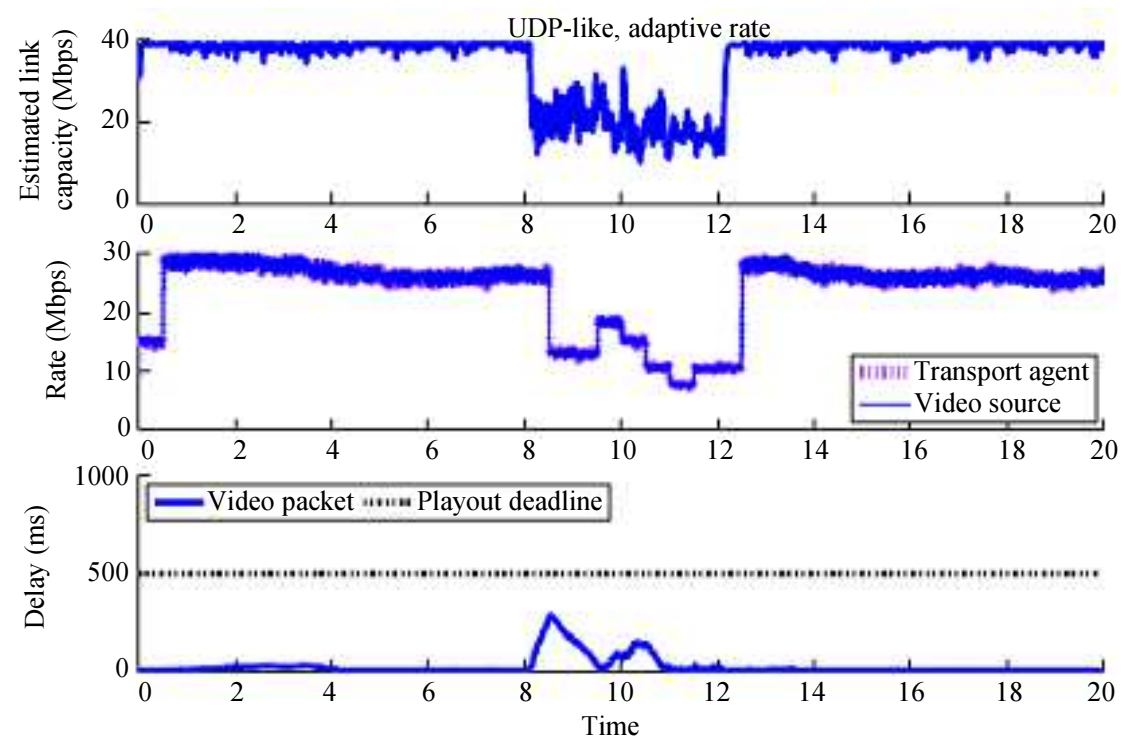

(b)

Fig. 41: Comparison between video streaming over a single wireless link: (a) fixed video source rate over traditional TCP; (b) adaptive video rate via bit stream switching over a video-aware application-layer transport protocol, with reduced ACK frequency (Courtesy of (Zhu and Girod, 2007))

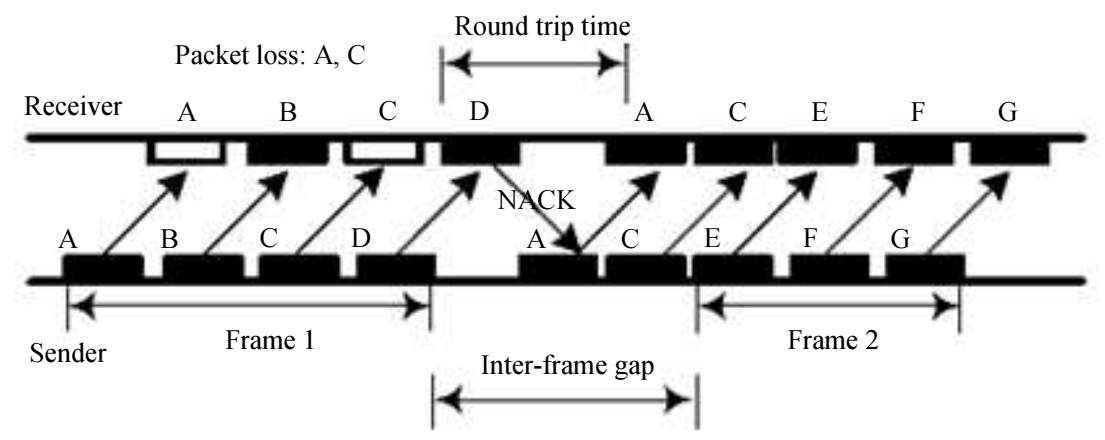

Fig. 42: The Inter-frame Retransmission (IR) NACK (Courtesy of (Al-Akaidi and Hamzaoui, 2011)) 


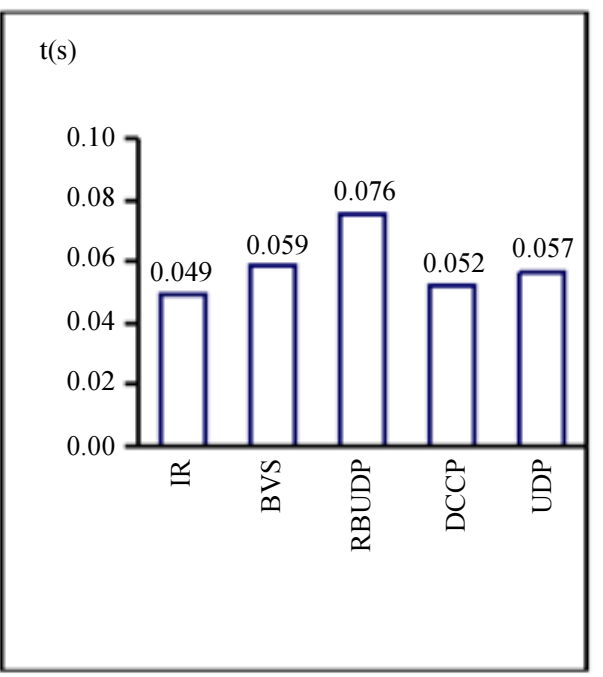

(a)

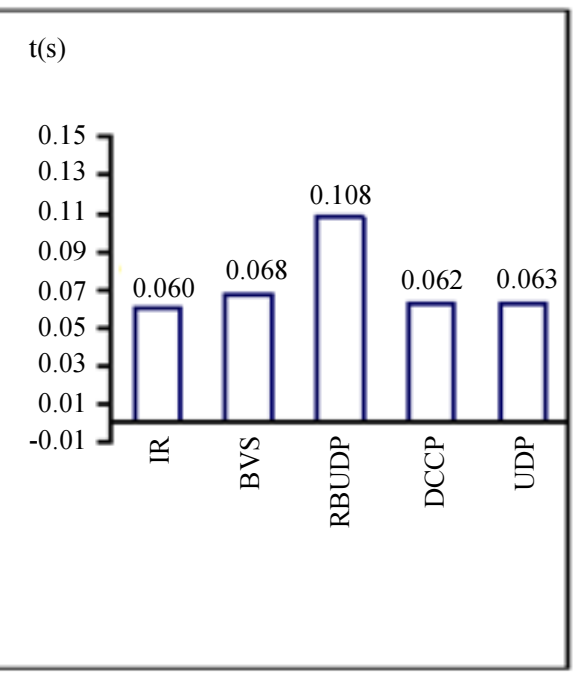

(b)

Fig. 43: Comparing IR with other protocols by using average (packet and frame) delay (Courtesy of (Al-Akaidi and Hamzaoui, 2011)) (a) Average packet delay (b) average frame delay

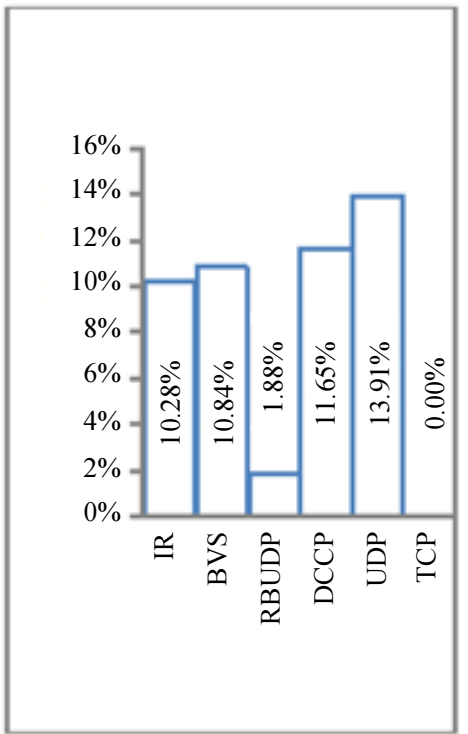

(a)

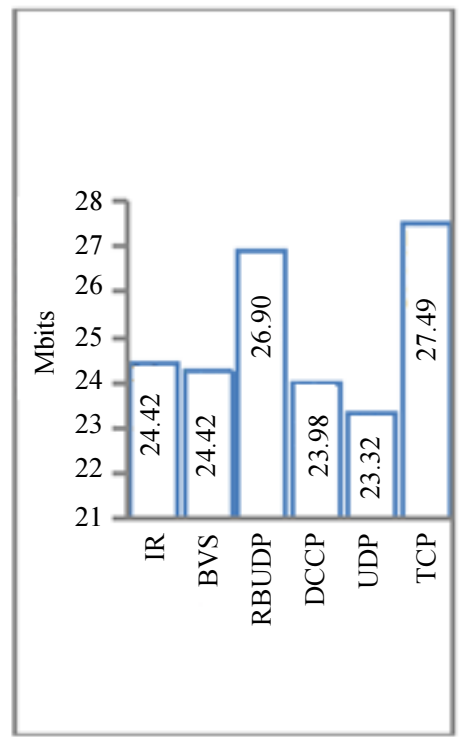

(b)

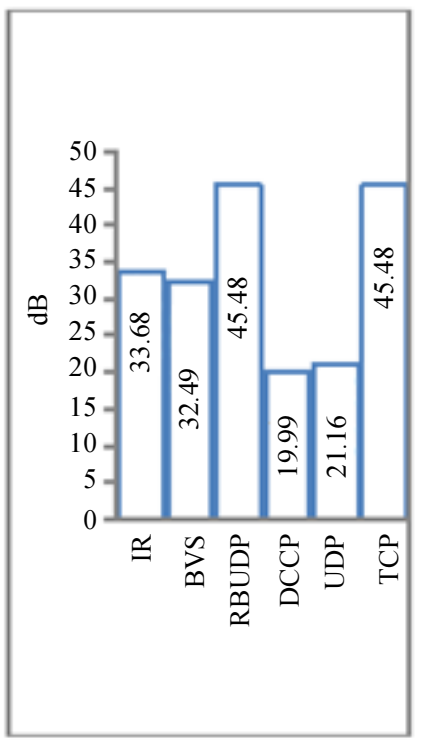

(c)

Fig. 44: Comparing IR with other protocols by using packet loss rate, throughput and PSNR (Courtesy of (Al-Akaidi and Hamzaoui, 2011)) (a) packet loss (b) cumulative throughput (c) PSNR

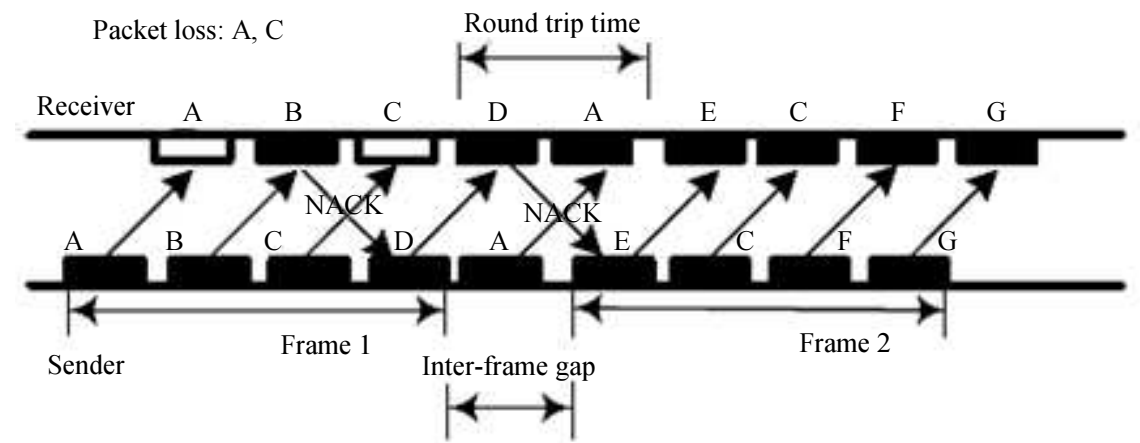

(a) 


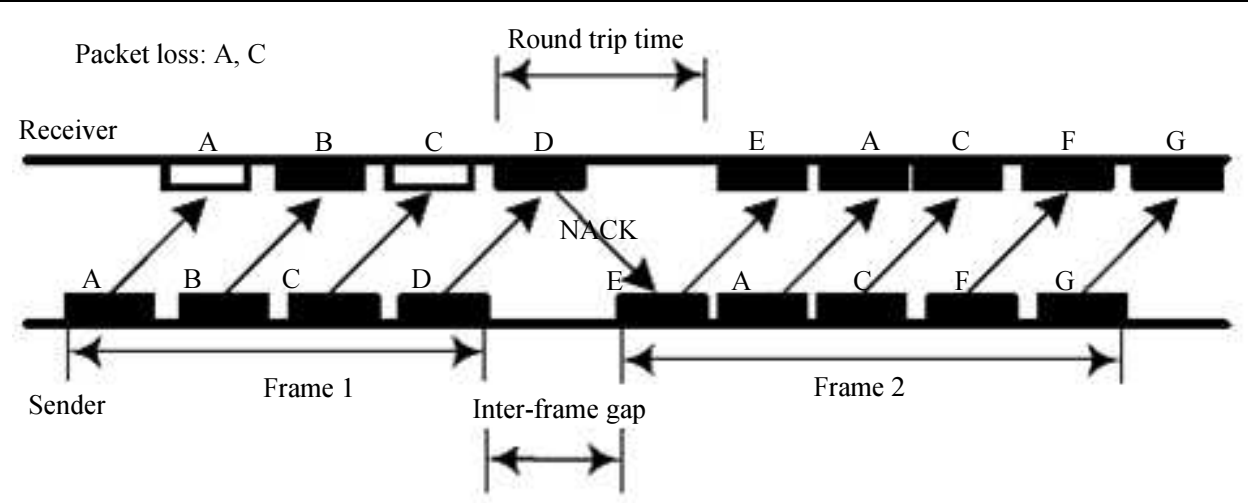

(b)

Fig. 45: Comparison between QR and IR when RTT > Inter Frame Gap (IFG) (Courtesy of (Al-Akaidi and Hamzaoui, 2011)) (a) quick response NACK when RTT $>$ IFG (b) inter-frame retransmission MACK when RTT $>$ IFG

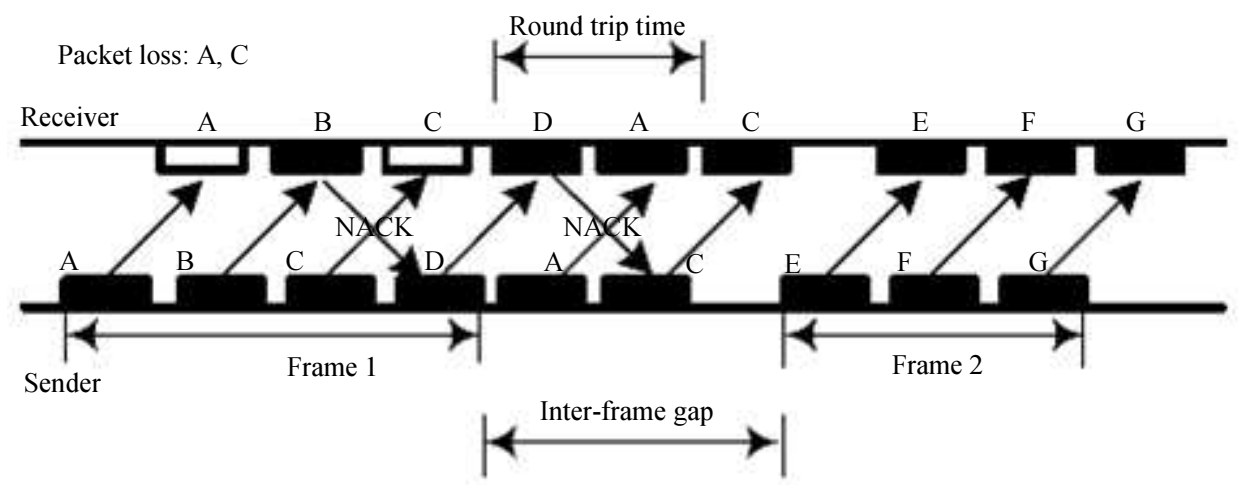

(a)

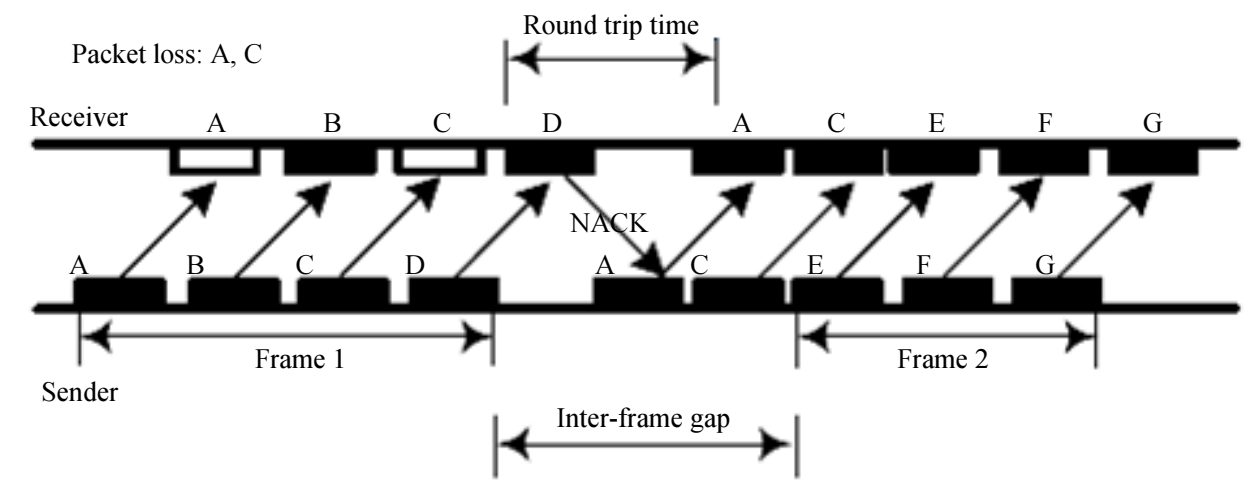

(b)

Fig. 46: Comparison between QR and IR when RTT < IFG (Courtesy of (Al-Akaidi and Hamzaoui, 2011)) (a) quick response NACK when RTT $<$ IFG (b) inter-frame retransmission NACK when $\mathrm{RTT}<\mathrm{IFG}$

In IR the receiver just send only one NACK for all the lost packets in one frame; Therefore IR generates less NACK packets than QR and this leads to a small delay and small number of interrupts, also it reduces the lost packets rate (as shown in Fig. 45 and 46). Finally we can say that the overall performance of IR is better than QR (as proved in (Al-Akaidi and Hamzaoui, 2011)).

\section{Comparison between IR and Prioritized IR}

The NACK packet strategy can be applied by sending NACK packet only for prioritized packets; consequently it leads to significant decrease in the packets and frames delays (by about 11 and $31 \mathrm{~ms}$ respectively). However, it increases the lost packets rates; also it reduces throughput and video quality (Fig. 47). By the way, the 


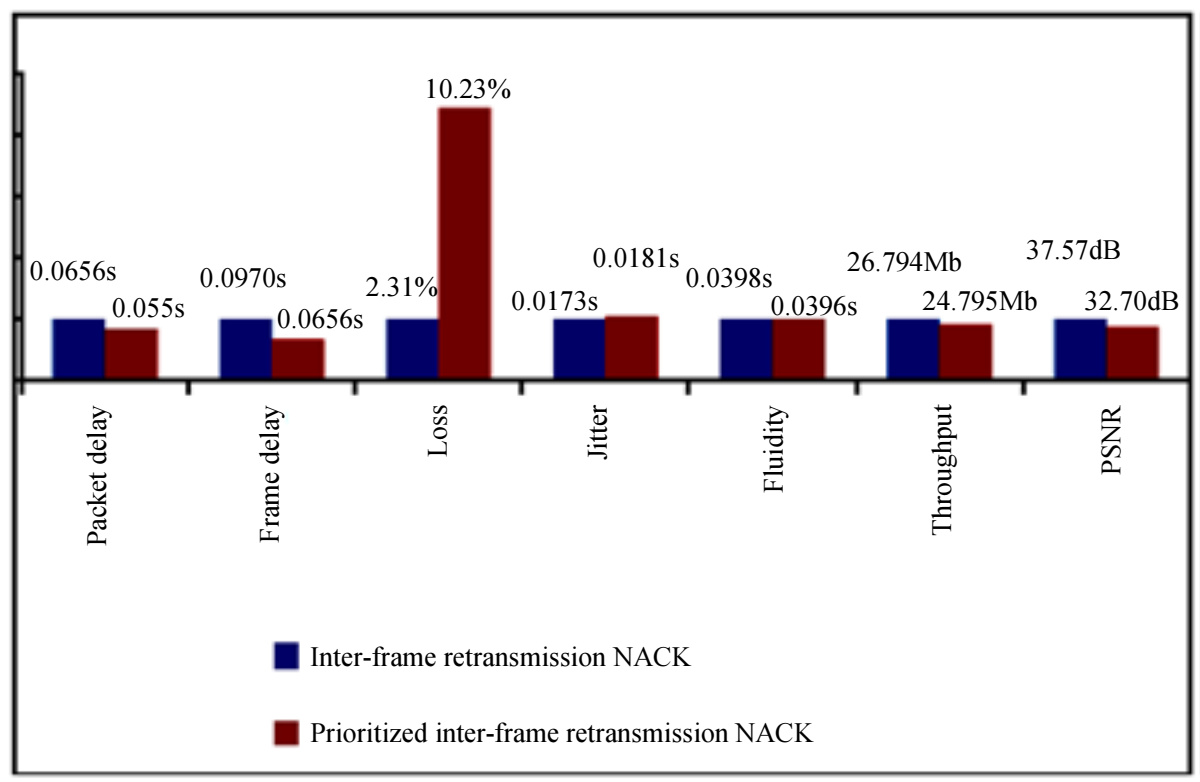

Fig. 47: The performance evaluation of IR vs. Prioritized IR (Courtesy of (Al-Akaidi and Hamzaoui, 2011))

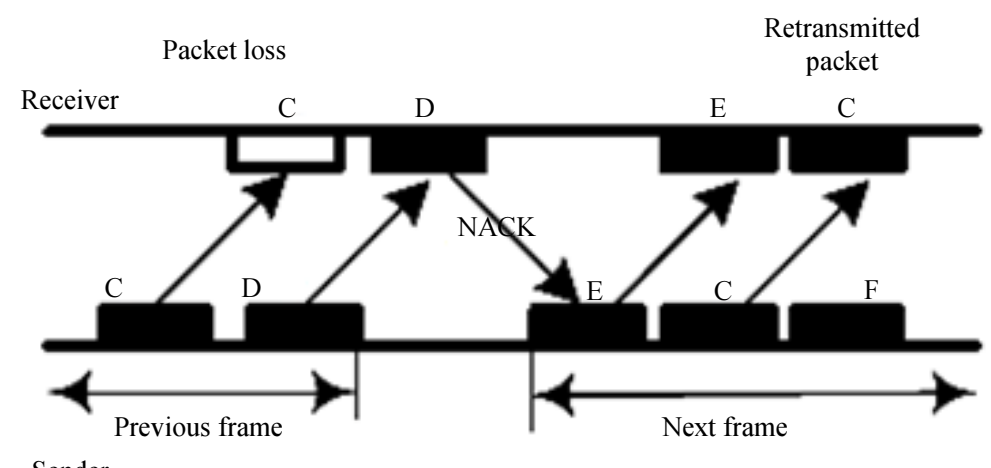

(a)

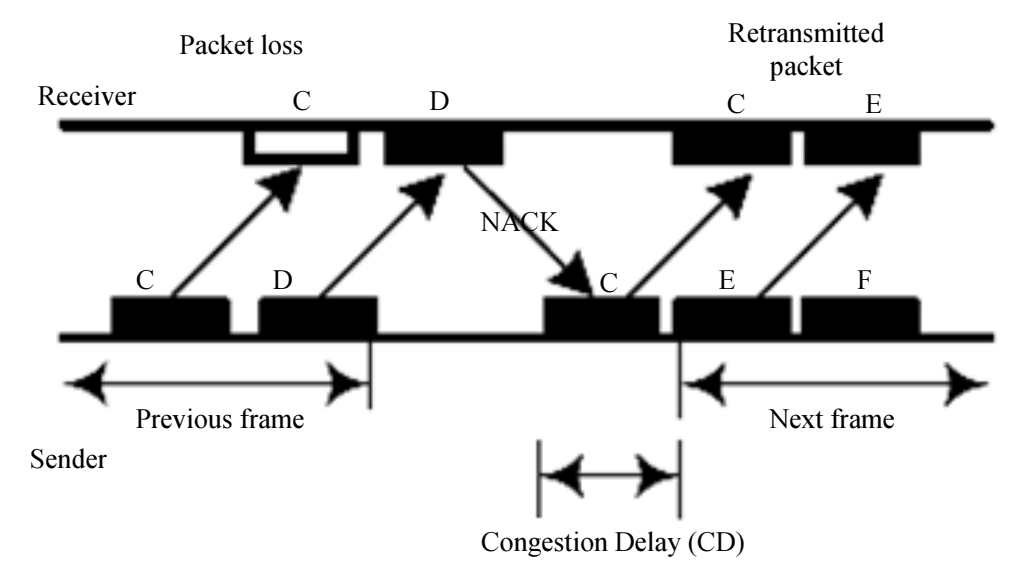

(b)

Fig. 48: Comparison between IR without CD and IR with CD (Courtesy of (Al-Akaidi and Hamzaoui, 2011)) (a) without Congestion Delay (CD) (b) with Congestion Delay (CD) 


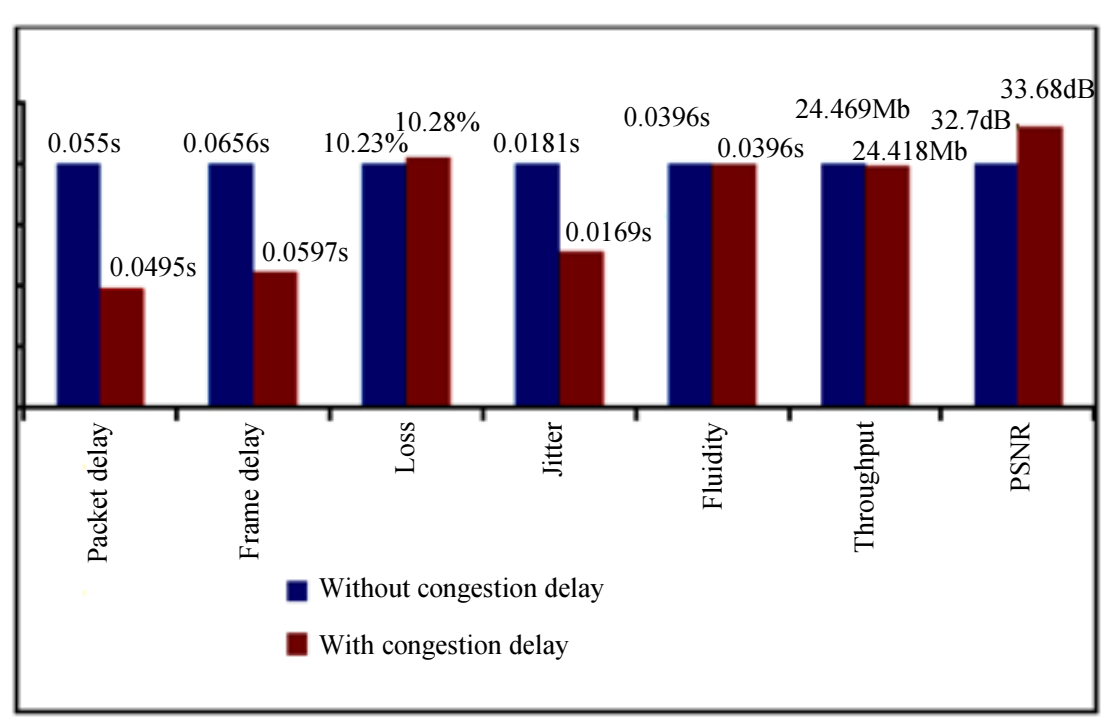

Fig. 49: The performance evaluation of Prioritized IR without CD vs. Prioritized IR with CD (Courtesy of (Al-Akaidi and Hamzaoui, 2011))

\section{Comparison between IR with Congestion Delay and IR without Congestion Delay}

In case if there are some lost packets in the current frame, then some Congestion Delay (CD) can be added to make sure that the current frame arrives before the next frame (Fig. 48) (Al-Akaidi and Hamzaoui, 2011).

Actually, adding $\mathrm{CD}$ technique reduces the average packet and frame delays (by about $6 \mathrm{mswhen} C \mathrm{CD}=1 \mathrm{~ms}$ ) in prioritized IR; Furthermore it reduces the average jitter (Fig. 49).

However, this technique leads to marginal increase in the packet loss rate, which decreases the cumulative throughput (as shown in Fig. 49). On the other hand, prioritized IR with $\mathrm{CD}$ which protects the prioritized frames leads to enhancing the video streaming quality (or PSNR) over WiMAX wireless network.

To conclude, the prioritized IR with CD helps in decreasing the average (packets and frames) delay by about $6 \mathrm{~ms}$ while it preserves the prioritized frames; Therefore it leads to enhancing the video streaming quality (or PSNR) over WiMAX wireless network.

\section{Packet Erasure Networks/Packet Erasure Channels}

Several researches consider the problem of erasure packets such as (Ranganathan et al., 2017) that finds the optimal rates of the erasure code and the channel code to minimize the transmit power required for a certain message error probability.

$\mathrm{Xu}$ et al. (2017) studies the achievable throughput of batched temporal network coding in multi-hop erasure channels, where network coding is applied only within small coding blocks and each communication hop is modeled as a Gilbert-Elliott (GE) packet erasure channel.

Amiri and Gündüz (2017) studies cache-aided broadcast network, in which a server delivers contents to a group of receivers over a packet erasure broadcast channel.

Ghorbel et al. (2015) considers a cache-enabled K-user broadcast erasure packet channel in which a server with a library of $\mathrm{N}$ files wishes to deliver a requested file to each user who is equipped with a cache of a finite memory $\mathrm{M}$.

Wang (2016) studies the 1-to-2 broadcast packet erasure channels with causal Acknowledgement (ACK), which is motivated by practical downlink access point networks.

Gerami and Xiao (2013) studies the repair problem of distributed storage systems in erasure networks where the packets transmitted from surviving nodes to the new node might be lost. The fundamental storage-bandwidth tradeoff is calculated by multicasting analysis in erasure networks. The optimal tradeoff bound can be asymptotically achieved when the number of transmission (packets) goes to infinity. Etezadi and Khisti (2012) studies real-time coding of Gauss-Markov sources over burst-erasure channels, it develops lower and upper bounds for the function and observe that the bounds coincide in the high resolution limit.

\section{Quality of Video Streaming and Applications in 4G Networks Compare to 3G Networks}

In the third generation $(3 \mathrm{G})$ and fourth generation (4G) wireless ecosystem, user devices such as iPhones and androids equipped with video capture capabilities are standard. Coupled with social media sites (Facebook, blogs), this results in the potential to cause huge growth 
in the capture and streaming of video from a mobile device. However, this potential is severely tempered due to the bandwidth limitation on the wireless uplink (Payette et al., 2011).

Chaurasia and Jagannatham (2013) proposes a new scalable hierarchical wavelet decomposition based layered video coding over parallel TCP scheme for reliable video transmission over MIMO wireless channels while reducing the delay and jitter of video streaming over $3 \mathrm{G}$ and $4 \mathrm{G}$ mobile networks.

\section{Summary}

In recent years, real-time and live video streaming become very important topics in the computer communication world. In this tutorial paper we presented the main ideas of video streaming and the most important methods for enhancing it over the wired and wireless packet erasure networks. First in section II, we presented an overview of video and video streaming history. Then in section III, we showed an overview of using compression standards in video-streaming. In section IV, we presented some error correction techniques that help in solving the congestion and lost packets problems in video streaming, also we showed the fixed (or static) rate Forward Error Correction (FEC) like traditional Reed-Solomon codes for enhancing the video streaming. However the fixed rate FEC techniques lead to bandwidth expansion (or increasing bandwidth overhead) and hence increase the lost packets rates, therefore this leads to reduce the Peak Signal to Noise Ratio (PSNR). Then in section V, we demonstrated the rateless FEC (e.g., Fountain codes) with feedbacks that help in reducing the FEC bandwidth overhead while keeping successful reconstruction of the video streaming and hence results in enhancing the PSNR and decreasing the congestion level. After that in section VI, we talked about the Unequal Error Protection (UEP) techniques which are more powerful and achieve better PSNR than the EEP techniques; also we presented some recent researches which proved that the UEP techniques are more suitable for the high quality video streaming applications. Then Section VII presented the Layered Video Streaming concepts. In section VIII, we clearly demonstrated the differences between single path and multiple paths video-streaming techniques. Finally, in section IX we focused on some new researches that based on hybrid solutions between advanced BEC and rateless FEC techniques to solve the real-time and live video streaming issues over $3 \mathrm{G}$, Wi-Fi and WiMAX wireless networks.

\section{Conclusion}

We introduced tutorial paper that can help computer/communication engineering students and new researchers to improve their knowledge and have sufficient information on video streaming and the most important methods for enhancing its quality over wired and wireless packets networks. From sub-section III.G and due to the scalability, low decoding complexity (especially in Scalable Baseline profile and low level of the Scalable High profile) and good R-D performance of H.264/SVC; therefore it can be concluded that H.264/SVC (layered coding) is considered one of the best video compression standards to deal with the realtime and live video streaming applications. However, in sub-section III.H we found that HEVC leads to significant decrease in bit-rate by approximately $50 \%$ while keeping the same level of perceptual video quality as in the previous standards. Furthermore, it can be concluded that using rateless FEC (e.g. LT and Raptor codes) coding techniques with feedbacks (based on the packet loss rate histogram) leads to decreasing the FEC coding bandwidth overhead while keeping good reconstruction of the live video streaming over the packet erasure networks (refer to sub-section V.D) and hence results in decreasing the packet loss rate, decreasing the congestion level and as a result enhancing the performance of video streaming (like enhancing the average PSNR). Also we strongly believe on that using the static UEP technique (in subsection VI.C) achieves a better average PSNR performance than the EEP techniques. However, in sub-section VI.D we show that adaptive UEP technique outperforms the static UEP technique significantly in terms of average PSNR. Therefore adaptive UEP technique is more suitable for the applications where high video quality is required. Also from section VI, it can be concluded that applying UEP with rateless FEC codes toMPEG-2and H.264/SVC video compression standards leads to better PSNR than using EEP with rateless codes in MPEG-2 and H.264/SVC. From subsection VII.C it can be concluded that the Recent UEP scheme (see sub-section VI.C) achieved better PSNR performance (in H.264/SVC layered coding compression standard) than the previous UEP schemes, Also the encoding and decoding time of recent UEP scheme were still low enough and suitable for Broadcast/Multicast real-time video streaming applications. On the other hand, applying multipath video streaming from many senders to single receiver (in sub-section VIII.B.ii) leads to better loss characteristics than single path video streaming with or without the use of an erasure code. Furthermore, the overall load on the network will not be increased using this approach. Also multipath video streaming leads to better load distribution on the network than single path video streaming. Moreover, we strongly believe on applying flexible Distributed Rateless Codes with UEP technique (DU-rateless codes) to the multiple senders 
and single receiver method (in sub-section VIII.B.ii) leads to better PSNR with minimal error rates. Finally, from sub-section IX.F.iii it can be concluded that the prioritized IR with CD helps in decreasing the average (packets and frames) delay and preserving the prioritized frames; Therefore it leads to enhancing the video streaming quality (or PSNR) over WiMAX wireless network.

\section{Acknowledgment}

This research was supported and funded by Arab Open University - Kuwait Branch. We thank everybody who assisted us to improve the study.

\section{Author's Contributions}

Both authors, Dr. Yahia Hasan And Mr. Ali Zanbouri, participated in all stages of research: coordination, analysis, designing the research plan, organizing the study and contributing to the writing of the manuscript.

\section{Ethics}

We testify that this research paper submitted to the Journal of Science Publication, title: State of the Art on Video Streaming and Applications has not been published in whole or in part elsewhere.

This research project was conducted with full compliance of research ethics norms of Arab Open University - Kuwait.

\section{References}

3GPP, 2005. The mobile broadband standard.

Abdouni, B., W.C. Cheng, A.L.H. Chow, L. Golubchik and J.C.S. Lui, 2005. Multi-path streaming: Optimization and evaluation. SPIE Proc., 5680: 254-265.

Abomhara, M., O.O. Khalifa, O. Zakaria, A.A. Zaidan and B.B. Zaidan et al., 2010. Video compression techniques: An overview. J. Applied Sci., 10: 1834-1840. DOI: 10.3923 /jas.2010.1834.1840

ACC, 2008. White paper: An explanation of video compression techniques. Axis Communication Company.

Afzal, J., T. Stockhammer, T. Gasiba and W. Xu, 2006. Video streaming over MBMS: A System design approach. J. Multimedia, 1: 25-35.

Ahmad, S., R. Hamzaoui and M. Al-Akaidi, 2007. Robust live unicast video streaming with rateless codes. Proceedings of the 16th International Packet Video Workshop, Nov. 12-13, IEEE Xplore Press, Lausanne, Switzerland, pp: 78-84.

DOI: 10.1109/PACKET.2007.4397028
Ahmad, S., R. Hamzaoui and M. Al-Akaidi, 2008a. Practical channel-adaptive video streaming with fountain codes. Proceedings of the 3rd International Conference on Pervasive Computing and Applications, Oct. 6-8, IEEE Xplore Press, Alexandria, Egypt, pp: 351-355. DOI: 10.1109/ICPCA.2008.4783608

Ahmad, S., R. Hamzaoui and M. Al-Akaidi, 2008b. Unequal error protection using LT codes and block duplication. Proceedings of the 9th Middle Eastern Multiconference on Simulation and Modelling, Aug. 26-28, Amman.

Ahmad, S., R. Hamzaoui and M. Al-Akaidi, 2009. Video multicast using unequal error protection with Luby Transform codes. Proceedings of the Middle Eastern Multi Conference on Simulation and Modelling, (CSM' 09), Beyrouth.

Ahmad, S., R. Hamzaoui and M. Al-Akaidi, 2010. Adaptive unicast video streaming with rateless codes and feedback. IEEE Trans. Circuits Syst. Video Technol., 20: 275-285. DOI: 10.1109/TCSVT.2009.2031545

Ahmad, S., R. Hamzaoui and M. Al-Akaidi, 2011. Unequal error protection using Fountain codes with applications to video communication. IEEE Trans. Multimedia, 13: 92-101. DOI: 10.1109/TMM.2010.2093511

Akabri, B., H.R. Rabiee and M. Ghanbari, 2007. Packet loss recovery schemes for peer-to-peer video streaming. Proceedings of the 3rd International Conference on Networking and Services, Jun. 1925, IEEE Xplore Press, Athens, Greece, pp: 94-94. DOI: $10.1109 /$ ICNS.2007.91

Al-Akaidi, S.M. and R. Hamzaoui, 2011. Inter-frame retransmission for video surveillance over WIMAX. Proceedings of the Middle Eastern Multi Conference on Simulation and Modelling, (CSM' 11), Amman.

Amiri, M.M. and D. Gündüz, 2017. Cache-aided content delivery over erasure broadcast channels. IEEE Trans. Commun., 66: 370-381.

DOI: 10.1109/TCOMM.2017.2751608

Apostolopoulos, J., T. Wong, W. Tan and S. Wee, 2002. On multiple description streaming with content delivery networks. Proceedings of the 21th Annual Joint Conference of the IEEE Computer and Communications Societies, Jun. 23-27, IEEE Xplore Press, USA, pp: 1736-1745.

DOI: 10.1109/INFCOM.2002.1019427

Bova, T. and T. Krivoruchka, 1999. Realiable UDP protocol. IETF Draft.

Caro, D., 2004. Wireless versus wired networks: A notable problem with wireless devices is that they still need a power source. 
Chaurasia, A.K. and A.K. Jagannatham, 2013. Dynamic parallel TCP for scalable video streaming over MIMO wireless networks. Proceedings of the 6th Joint IFIP Wireless and Mobile Networking Conference, Apr. 23-25, IEEE Xplore Press, Dubai, UAE, pp: 1-6. DOI: 10.1109/WMNC.2013.6549058

Chou, C.M., C.Y. Li, W.M. Chien and K.C. Lan, 2009. A feasibility study on vehicle-to-infrastructure communication: WiFi Vs. WiMAX. Proceedings of the 10th International Conference on Mobile Data Management: Systems, Services and Middleware, May 18-20, IEEE Xplore Press, Taipei, Taiwan, pp: 397-398. DOI: 10.1109/MDM.2009.127

Chow, A.L.H., L. Golubchik and J.C.S. Lui, 2005. Toward picture-perfect streaming on the internet. Proceedings of the 2nd International Conference on the Quantitative Evaluation of Systems, Sept. 19-22, IEEE Xplore Press, Torino, Italy, pp: 63-72.

DOI: 10.1109/QEST.2005.41

Daintith, J., 2004. Forward error correction: A Dictionary of Computing. 5th Edn., Oxford University Press, New York.

Dhawan, S., 2007. Analogy of promising wireless technologies on different frequencies: Bluetooth, $\mathrm{WiFi}$ and WiMAX. Proceedings of the 2nd International Conference on Wireless Broadband and Ultra Wideband Communications, Aug. 27-30, IEEE Xplore Press, Sydney, NSW, Australia, pp: 14-14. DOI: 10.1109/AUSWIRELESS.2007.27

Elaywe, A.H. and A.E. Kamal, 2002. TCP with Source Traffic Shaping (TCP-STS) an approach to congestion reduction in the internet. SPIE.

Etezadi, F. and A. Khisti, 2012. Real-time coding of GaussMarkov sources over burst erasure channels. Proceedings of the 50th Annual Allerton Conference on Communication, Control and Computing (Allerton), IEEE Xplore Press, Monticello, IL, USA, pp: 2037-2044. DOI: 10.1109/Allerton.2012.6483473

FFmpeg. Available: http://ffmpeg.mplayerhq.hu/.

Floyd, S. and V. Jacobson, 1993. Random early detection gateways for congestion avoidance. IEEE/ACM Trans. Network., 1: 397-413. DOI: $10.1109 / 90.251892$

Foley, P., 1996. The Mpact media processor redefines the multimedia PC. Proceedings of the 'Technologies for the Information Superhighway' Digest of Papers, Feb. 25-28, IEEE Xplore Press, Santa Clara, CA, USA, pp: 311-318. DOI: $10.1109 /$ CMPCON.1996.501789

Franceschinis, G., C. Anglano, L. Golubchik, J.C.S. Lui and T.F. Tung et al., 2002. Multi-path continuous media streaming: what are the benefits? Performance Evaluat., 49: 429-449.

DOI: $10.1016 / \mathrm{S} 0166-5316(02) 00125-6$

FU, 2011. Real Networks Inc., History.
Gallucci, D., A. Fiandrotti, E. Masala and J.C. De Martin, 2009. Content-adaptive robust H.264/SVC video communications over 802.11e networks. Proceedings of the International Conference on Advanced Information Networking and Applications, May 26-29, IEEE Xplore Press, Bradford, UK, pp: 189-196.

DOI: 10.1109 /AINA.2009.90

Gerami, M. and M. Xiao, 2013. Repair for distributed storage systems with erasure channels. Proceedings of the IEEE International Conference on Communications, Jun. 9-13, IEEE Xplore Press, Budapest, Hungary, pp: 4058-4062.

DOI: $10.1109 /$ ICC.2013.6655195

Ghareeb, M., 2008. About multiple paths videostreaming: State of the art. Publication Interne $\mathrm{N}^{\circ}$ 1905, Inria-00336306, Version.

Ghorbel, A., M. Kobayashi and S. Yang, 2015. Cacheenabled broadcast packet erasure channels with state feedback. Proceedings of the 53rd Annual Allerton Conference on Communication, Control and Computing, Sept. 29-Oct. 2, IEEE Xplore Press, Monticello, IL, USA, pp: 1446-1453. DOI: 10.1109/ALLERTON.2015.7447178

Golubchik, L. and J.C.S. Lui, 2002. Multi-path streaming: Is it worth the trouble. ACM SIGMETRICS Performance Evaluat. Rev., 30: 12-14. DOI: $10.1145 / 605521.605527$

Greaves, D. and M. Taunton, 1996. ATM for video and audio on demand. Proceedings of the AES Conference in London, (CL' 96).

Hamzaoui, R., S. Ahmad and M. Al-Akaidi, 2008. Reliable wireless video streaming with digital fountain codes. Encyclopedia of Wireless and Mobile Communications.

He, E., J. Leigh, O. Yu and T. DeFanti, 2002. Reliable Blast UDP: Predictable high performance bulk data transfer. Proceedings of the IEEE International Conference on Cluster Computing, Sept. 26-26, IEEE Xplore Press, Chicago, IL, USA, pp: 317-324. DOI: 10.1109/CLUSTR.2002.1137760

Ibáñez, S.R., R.A. Santos, V.R. Licea, A.E. Block and M.Á.G. Ruiz, 2008. Hybrid WiFi-WiMAX network routing protocol. Proceedings of the Electronics, Robotics and Automotive Mechanics Conference, Sept. 30-Oct. 3, IEEE Xplore Press, pp: 87-92.

DOI: $10.1109 /$ CERMA.2008.24

ITU, 2008. Cellular standards for the third generation.

Jian-Wen, C., K. Chao-Yang and L. Youn-Long, 2006. Introduction to H.264 advanced video coding. Proceedings of the Asia and South Pacific Conference on Design Automation, Jan. 24-27, IEEE Xplore Press, Yokohama, Japan, pp: 736-741. DOI: 10.1109/ASPDAC.2006.1594774 
Kamal, A.E., 2004. Discrete-time modeling of TCP Reno under background traffic interference with extension to RED-based routers. Performance Evaluat., 58: 109-142. DOI: 10.1016/j.peva.2004.07.007

Kuroda, I. and T. Nishitani, 1998. Multimedia processors. Proc. IEEE, 86: 1203-1221. DOI: 10.1109/5.687835

Lee, K.W., S. Ha, J.R. Li and V. Bharghavan, 2000. An application-level multicast architecture for multimedia communications. Proceedings of the 8th ACM International Conference on Multimedia, Oct. 30-Nov. 03, ACM, Los Angeles, CA, USA, pp: 398-400. DOI: 10.1145/354384.354544

Li, X. and M.H. Ammar, 1996. Bandwidth control for replicated-stream multicast video distribution. Proceedings of the 5th IEEE International Symposium on High Performance Distributed Computing, Aug. 6-9, IEEE Xplore Press, Syracuse, NY, USA, pp: 356-363.

DOI: 10.1109/HPDC.1996.546206

Li, X., S. Paul and M. Ammar, 1998. Layered Video Multicast with Retransmissions (LVMR): Evaluation of hierarchical rate control. Proceedings of the 17th Annual Joint Conference of the IEEE Computer and Communications Societies, Mar. 29Apr. 2, IEEE Xplore Press, San Francisco, CA, USA, pp: 1062-1072.

DOI: 10.1109/INFCOM.1998.662916

Liu, Y., S. Zhang, S. Xu, Y. Zhang and Y. Liu, 2009b. Research on H.264/SVC compressed video communication in 3G. Proceedings of the 4th International Conference on Computer Science and Education, Jul. 25-28, IEEE Xplore Press, Nanning, China, pp: 327-332.

DOI: 10.1109/ICCSE.2009.5228433

Liu, Z., Y. Shen, K.W. Ross, S.S. Panwar and Y. Wang, 2009a. LayerP2P: Using layered video chunks in P2P live streaming. IEEE Trans. Multimedia, 11: 1340-1352. DOI: 10.1109/TMM.2009.2030656

Luby, M., 2002. LT Codes. Proceedings of the 43rd Annual IEEE Symposium on Foundations of Computer Science, Nov. 19-19, IEEE Xplore Press, Vancouver, BC, Canada. DOI: $10.1109 /$ SFCS.2002.1181950

Lun, D.S., N. Ratnakar, M. Médard, R. Koetter and D.R. Karger et al., 2006. Minimum-cost multicast over coded packet networks. IEEE Trans. Inform. Theory, 52: 2608-2623.

DOI: $10.1109 /$ TIT.2006.874523

MacKay, D.J.C., 2005. Capacity approaching codes design and implementation. IEE Proc. Commun., 52: 1060-1061. DOI: 10.1049/ip-com:20059077

Mahdavi, J. and S. Floyd, 1997. TCP-friendly unicast rate-based flow control. Technical Note Sent to the end2end-Interest Mailing List.
Martinez, J.M., 2002. Standards - MPEG-7 overview of MPEG-7 description tools, Part2. IEEE Multimedia, 9: 83-93. DOI: 10.1109/MMUL.2002.1022862

Mohr, A.E., E.A. Riskin and R.E. Ladner, 2000. Unequal loss protection: Graceful degradation of image quality over packet erasure channels through forward error correction. IEEE J. Selected Areas Commun., 18: 819-828. DOI: 10.1109/49.848236

Mueller, S., 1992. Upgrading and Repairing PCs. 2nd Edn., Que Books, ISBN-10: 0-88022-856-3, pp: 669-692.

Nguyen, T. and A. Zakhor, 2002. Distributed video streaming with forward error correction. This work was supported by NSF under grants CCR-9979442 and ANI-9905799 and by AFOSR contract F4962000-1-0327.

Padhye, J., J. Kurose and D. Towsley, 1999. A TCPfriendly rate adjustment protocol for continuous media flows over best effort networks. This Material is Based Upon Work Supported by the National Science Foundation.

Payette, C., B. Cilli, Z. Sayeed and S. Sharma, 2011. Enabling reliable and efficient wireless video uplink. Bell Labs Tech. J., 16: 169-191.

DOI: $10.1002 /$ bltj.20509

Phifer, L.A., 2010. Delivering video over your 802.11n wireless LAN.

Pishro-Nik, H., N. Rahnavard and F. Fekri, 2005. Nonuniform error correction using low-density parity-check codes. IEEE Trans. Inform. Theory, 51: 2702-2714. DOI: 10.1109/TIT.2005.850230

Pitchaipillai, P. and K. Eswaramoorthy, 2017. H.264/MPEG-4 AVC video streaming evaluation of LR-EE-AOMDV protocol in MANET. CIT. J. Comput. Inform. Technol., 25: 15-29. DOI: $10.20532 /$ cit.2017.1003320

Polsson, K., 2010. Chronology of IBM Personal Computers.

Puri, A., X. Chen and A. Luthra, 2004. Video coding using the H.264/MPEG-4 AVC compression standard. Signal Process.: Image Commun., 19: 793-849. DOI: 10.1016/j.image.2004.06.003

Puri, R., K.W. Lee, K. Ramchandran and V. Bharghavan, 1997. An integrated source transcoding and congestion control paradigm for video streaming in the internet. IEEE Trans. Multimedia, 3: 18-32. DOI: 10.1109/6046.909591

Rahnavard, N. and F. Fekri, 2006. New results on unequal error protection using LDPC codes. IEEE Commun. Lett., 10: 43-45. DOI: 10.1109/LCOMM.2006.1576564

Rahnavard, N., B.N. Vellambi and F. Fekri, $2007 \mathrm{~b}$. Rateless codes with unequal error protection property. IEEE Trans. Inform. Theory, 53: 1521-1532. DOI: 10.1109/TIT.2007.892814 
Rahnavard, N., H. Pishro-Nik and F. Fekri, 2007a. Unequal error protection using partially regular LDPC codes. IEEE Trans. Commun., 55: 387-391. DOI: 10.1109/TCOMM.2007.892436

Ranganathan, S.V.S., T. Mu and R.D. Wesel, 2017. Allocating redundancy between erasure coding and channel coding when fading channel diversity grows with codeword length. IEEE Trans. Commun., 65: 3226-3237.

DOI: $10.1109 /$ TCOMM.2017.2706728

Rathnam, S. and G. Slavenburg, 1996. An architectural overview of the programmable multimedia processor, TM-1. Proceedings of the 'Technologies for the Information Superhighway' Digest of Papers, Fev. 25-28, IEEE Xplore Press, Santa Clara, CA, USA, pp: 319-326.

DOI: 10.1109/CMPCON.1996.501790. DOI:

Rejaie, R., M. Handley and D. Estrin, 2000. Layered quality adaptation for internet video streaming. IEEE J. Selected Areas Commun., 18: 2530-2543. DOI: $10.1109 / 49.898735$

Ribeiro, B., E. de Souza e Silva and D. Towsley, 2005. On the efficiency of path diversity for continuous media applications. Computer Science Technical Report 05-19, Department of Computer Science, University of Massachusetts, Amherst.

Richardson, I., 2007-2011. VcodexWhite Paper: An overview of H.264 advanced video coding.

Rodriguez, E., 2011. Wired Vs. wireless.

Santos-González, I., A. Rivero-García, J. Molina-Gil and P. Caballero-Gil, 2017. Implementation and analysis of real-time streaming protocols. Sensors, 17: 846-846. DOI: 10.3390/s 17040846

Schulzrinne, H. and S. Casner, 1996. RTP - a transport protocol for real-time applications. Internet Engineering Task Force.

Schwarz, H., D. Marpe and T. Wiegand, 2007. Overview of the scalable video coding extension of the H.264/AVC standard. IEEE Trans. Circuits Syst. Video Technol., 17: 1103-1120.

DOI: 10.1109/TCSVT.2007.905532

Sejdinovic, D., D. Vukobratovic, A. Doufexi, V. Senk and R. Piechocki, 2007. Expanding window fountain codes for unequal error protection. Proceedings of the 41st Asilomar Conference on Signals, Systems and Computers, Nov. 4-7, IEEE Xplore Press, Pacific Grove, CA, USA, pp: 1020-1024. DOI: 10.1109/ACSSC.2007.4487375

Shokrollahi, A., 2003. Raptor codes. Digital Fountain, Inc.

Shokrollahi, A., 2006. Raptor codes. IEEE Trans. Inf. Theory, 52: 2551-2567.

DOI: 10.1109 /TIT.2006.874390
Sisalem, D. and H. Schulzrinne, 1998. The loss-delay based adjustment algorithm a TCP-friendly adaption scheme. This Work was Funded in Part by the BMBF (German Ministry of Educationand Rese arch) and the DFN (German Research Network).

Sukanen, J., 2002. Peer-to-peer Communication. Residential and Virtual Home Environments Seminar on Internetworking.

Sullivan, G.J., J.R. Ohm, W.J. Han and T. Wiegand, 2012. Overview of the High Efficiency Video Coding (HEVC) standard. IEEE Trans. Circuits Syst. Video Technol., 22: 1649-1668. DOI: 10.1109/TCSVT.2012.2221191

Talari, A. and N. Rahnavard, 2009. Unequal error protection rateless coding for efficient mpeg video transmission. Proceedings of the IEEE Military Communications Conference, Oct. 18-21, IEEE Xplore Press, Boston, Boston, MA, USA, pp: 1-7. DOI: 10.1109/MILCOM.2009.5379795

Talari, A. and N. Rahnavard, 2010. Distributed rateless codes with UEP property. Proceedings of the IEEE International Symposium on Information Theory Proceedings, Jun. 13-18, IEEE Xplore Press, Austin, TX, USA, pp: 2453-2457.

DOI: $10.1109 /$ ISIT.2010.5513758

The History of Video and Related Innovations. http://inventors.about.com/library/inventors/blvideo. htm

Tosun, A.S., 2009. Videocompression: MPEG-4 and beyond.

Tourapis, A.M., K. Sühring and G. Sullivan, 2005. Revised H.264/MPEG-4 AVC reference software manual. Joint Video Team, Doc. JVT-Q042.

TSGSSA, 2008. Multimedia Broadcast/Multicast Service (MBMS); Protocols and codecs. 3GPP TS 26.346 V7.6.0 Release 7, Technical Specification Group Services and System Aspects.

Wang, C., D. Sklar and D. Johnson, 2002. Forward error-correction coding. crosslink-the aerospace corporation magazine of advances in aerospace technology (The Aerospace Corporation).

Wang, C.C., 2016. Delay-constrained capacity for broadcast erasure channels: A linear-coding-based study. IEEE Proceedings of the International Symposium on Information Theory, Jul. 10-15, IEEE Xplore Press, Barcelona, Spain, pp: 2903-2907. DOI: 10.1109/ISIT.2016.7541830

Wicker, S.B. and V.K. Bhargava, 1999. Reed-Solomon Codes and their Applications. 1st Edn., John Wiley and Sons, ISBN-10: 0780353919, pp: 336.

Wien, M., R. Cazoulat, A. Graffunder, A. Hutter and P. Amon, 2007. Real-time system for adaptive video streaming based on SVC. IEEE TCSVT, 17: 1227-1237. DOI: 10.1109/TCSVT.2007.905519 
Xu, K., M. Gerla, L. Qi and Y. Shu, 2003. Enhancing TCP fairness in ad hoc wireless networks using neighborhood RED. Proceedings of the 9th Annual International Conference on Mobile Computing and Networking, Sept. 14-19, ACM, San Diego, CA, USA, pp: 16-28. DOI: $10.1145 / 938985.938988$

Xu, K., M. Gerla, L. Qi and Y. Shu, 2005. TCP unfairness in ad hoc wireless networks and a neighborhood RED solution. ACM MobiCom, 11: 383-399. DOI: $10.1007 / \mathrm{s} 11276-005-1764-1$

$\mathrm{Xu}$, X., Y.L. Guan and Y. Zeng, 2017. Batched network coding with adaptive recoding for multi-hop erasure channels with memory. IEEE Trans. Commun., 66: 1042-1052. DOI: 10.1109/TCOMM.2017.2765641
Zanbouri, A., M. Al-Akaidi and S. Ahmad, 2014. Adaptive unequal error protection to enhance multicast live video streaming. Proceedings of the Middle Eastern Multi Conference on Simulation and Modelling, (CSM' 14), Muscat.

Zhu, X. and B. Girod, 2007. Video streaming over wireless networks. Proceedings of the 15th European Signal Processing Conference, Sept. 3-7, IEEE Xplore Press, Poznan, Poland. 